\title{
The Weak Decay of Helium Hypernuclei
}

by

DOE/ER/40315--196

DE93 003650

\author{
Michael J. Athanas
}

A dissertation submitted in partial fulfillment of

the requirements for the degree of

\author{
Doctor of Philosophy
}

August 1992

\section{Carnegie Mellon University}

\section{DISCLAIMER}

This report was prepared as an account of work sponsored by an agency of the United States Government. Neither the United States Government nor any agency thereof, nor any of their employees, makes any warranty, express or implied, or assumes any legal liability or responsibility for the accuracy, completeness, or usefulness of any information, apparatus, product, or process disclosed, or represents that its use would not infringe privately owned rights. Reference herein to any specific commercial product, process, or service by trade name, trademark, manufacturer, or otherwise does not necessarily constitute or imply its endorsement, recommendation, or favoring by the United States Government or any agency thereof. The views and opinions of authors expressed herein do not necessarily state or reflect those of the United States Government or any agency thereof. 


\begin{abstract}
A $\Lambda$ hyperon replaces a neutron in a nucleus to form a hypernucleus via the ${ }^{A} X\left(K^{-}, \pi^{-}\right){ }_{\Lambda}^{A} X$ reaction at $750 \mathrm{MeV} / \mathrm{c}$ (Brookhaven Experiment 788). The free $\Lambda$ decay rates $\Gamma\left(\Lambda \rightarrow p \pi^{-}\right)$and $\Gamma\left(\Lambda \rightarrow n \pi^{\circ}\right)$ are diminished due to Pauli blocking but a non-mesonic decay mode, nucleon stimulated decay $N \Lambda \rightarrow N n$, is present and is detected via the energetic decay nucleon $(\mathrm{s})(\approx 400 \mathrm{MeV} / \mathrm{c})$. Measurements of the various hypernuclear decay rates $\Gamma\left(\Lambda \rightarrow p \pi^{-}\right), \Gamma\left(\Lambda \rightarrow n \pi^{\circ}\right), \Gamma(\Lambda n \rightarrow n n)$, and $\Gamma(\Lambda p \rightarrow n p)$ provides insight into the strong modification of the weak interaction such as the baryon-baryon $\Delta I=\frac{1}{2}$ rule. The hypernuclear state is isolated by momentum analysis of $\left(\mathrm{K}^{-}, \pi^{-}\right)$target reaction. Out-of-beam large volume scintillation detectors and tracking chambers are used to make particle identification of the hypernuclear decay products by time-of-flight, $d E / d x$, and range. The kinetic energy of the decay neutrons are measured by time of flight using the large volume 100 element neutron detector system. The hypernuclear lifetime is directly measured using precision scintillator counters and tracking chambers. Measurements of the various decay rates as well as the total lifetime are discussed for ${ }_{\Lambda}^{4} \mathrm{He}$.
\end{abstract}




\section{TABLE OF CONTENTS}

$\begin{array}{lll}\text { Chapter } 1 \text { Introduction } & 1\end{array}$

Introduction 1

1.1 Hypernuclei ....................... 1

1.2 The $\Lambda-N$ Weak Interaction ............... 2

1.2.1 Nuclear Physics Calculations ... . . . . . . . . . . 3

1.2.2 Isospin Structure of $\Delta \mathrm{S}=1$ Weak Hamiltonian . . . . . . . . 4

1.3 The $\Delta I=\frac{1}{2}$ Rule . . . . . . . . . . . . . . . 8

1.3 .1 Free $\Lambda$ Decay . . . . . . . . . . . . . . 9

1.3.2 The $\Delta I=\frac{1}{2}$ Rule and Hypernuclear Decay . . . . . . . . . 9

1.4 E788 ${ }_{\Lambda}^{4} \mathrm{He}$ Measurements ................. 15

$\begin{array}{lll}\text { Chapter } 2 \text { Apparatus } & 17\end{array}$

$\begin{array}{ll}\text { Apparatus } & 17\end{array}$

2.1 Experiment Beam Transport . . . . . . . . . . . . . 17

2.1.1 $K^{-}$and $\pi^{-}$Spectrometers ............. 19

2.2 Beamline Instrumentation . . . . . . . . . . . . 21

2.2 .1 In-Beam Tracking ................. 21

2.2.2 Beamline Counter Elements . . . . . . . . . . . 22

2.2.3 Trigger Level Electronics . . . . . . . . . . . . . . . 28

2.3 Cryogenic Liquid ${ }^{4} \mathrm{He}$ Target . . . . . . . . . . . . . . 28

2.3.1 Cryogenic Helium Target . . . . . . . . . . . . 28

2.3.2 Target Internal Veto System IV . . . . . . . . . . . 30

2.4 Target Out-of-Beam lounters . . . . . . . . . . . . . . . . 32

2.4.1 Target Veto Counters TV . . . . . . . . . . . . 32

2.4.2 Precision Target Scintillators SL/R . . . . . . . . . . . 33

2.4.3 Out-of-Beam Tracking Chambers ............ 35 
2.5 Out-of-Beam Detector Arrays . . . . . . . . . . . . . 37

2.5 .1 Layer One: $\mathrm{L} / \mathrm{RK} \ldots \ldots \ldots \ldots \ldots \ldots$

2.5 .2 Layer Two: $\mathrm{L} / \mathrm{RV} \ldots \ldots \ldots \ldots \ldots$

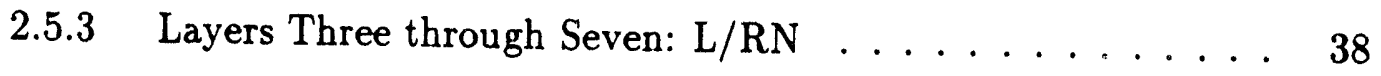

2.5 .4 Additional Elements $\ldots \ldots \ldots \ldots \ldots$

2.6 Measurement Electronics . . . . . . . . . . . . . . . . 39

$\begin{array}{lll}\text { Chapter } 3 \text { Analysis } & 45\end{array}$

Analysis

3.1 In-Beam Analysis $\ldots \ldots \ldots \ldots \ldots \ldots \ldots \ldots \ldots$

3.2 Data Reduction . . . . . . . . . . . . . 49

3.3 Out-of-Beam Detector Calibration . . . . . . . . . . 50

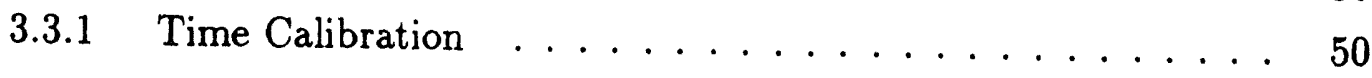

3.3.2 Energy Calibration . . . . . . . . . . . . . . 57

3.4 Decay Product Analysis $\ldots \ldots \ldots \ldots \ldots$

3.4 .1 Out-of-Beam Tracking $\ldots \ldots \ldots \ldots 60$

3.4.2 Charged Particle Identification . . . . . . . . . . 61

3.5 Detector Acceptance and Efficiency . . . . . . . . . . . 65

3.6 Target Veto Threshold Calibration . . . . . . . . . . 68

$\begin{array}{lll}\text { Chapter } 4 \text { Physics Results } & 77\end{array}$

Physics Results

4.1 Hypernuclear Lifetime Measurement . . . . . . . . . . . . 77

4.2 Decay Product Measurements . . . . . . . . . . . 86

4.2 .1 Total Hypernuclear Rate $\ldots \ldots \ldots \ldots$. . . . . . . . . . . . . . .

4.2 .2 Proton Measurement. . . . . . . . . . . . . . . 90

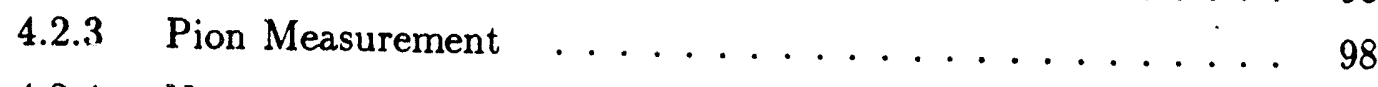

4.2.4 Neutron Measurement . . . . . . . . . . . . . 101

4.3 Rates and Normalization . . . . . . . . . . . . . . . . . . . . . . . . . . . . . . .

4.4 Partial Rates .................... . . 115 
$\begin{array}{lll}\text { Chapter } 5 \text { Discussion and Conclusion } & 117\end{array}$

Discussion and Conclusion $\quad 117$

5.1 General Discussion . . . . . . . . . . . . . . 117

5.1.1 Comparison to Previous Results . . . . . . . . 117

5.1.2 Implications for the $\Delta I=\frac{1}{2}$ Rule $\ldots \ldots \ldots \ldots \ldots 118$

5.1 .3 Future. . . . . . . . . . . . . . . 119

Appendix A The Isospin Antiquark Doublet in SU(2) 127

$\begin{array}{ll}\text { Bibliography } & 129\end{array}$ 


\section{The Weak Decay of Light $\Lambda$ Hypernuclei BNL Experiment 788}

M.J. Athanas ${ }^{1}$, P.D. Barnes ${ }^{2}$, G. Diebold ${ }^{3}$, G. Franklin, B. Quinn, F. Rozon ${ }^{4}$, R. Schumacher, I. Sukaton, V. Zeps

Carnegie Mellon University, Pittsburgh, PA.

S. Bart, R. Chrien, K. Johnson, P. Pile, R. Sutter, R. Sawafta Brookhaven National Laboratory, Upton, NY.

B. Bassalleck

University of New Mexico, Albuquerque, NM.

John Szymanski

Indiana University Cyclotron Facility, Bloomington IN

R. Stearns

Vassar College, Poughkeepsie, NY.

\section{Acknowledgements}

E. Cagalracan, Z. Conner, F. Merrill, D. Pierce, G. Vorinin, G. Wilkin, C. Maher, L. Addessi, B. Meier, E. Meier, J. Meier, A. Berdoz, V. Sum, A. Rusek, R. Zybert

${ }^{1}$ present address: University of California, San Diego, La Jolla, CA.

${ }^{2}$ present address: Los Alamos Laboratory, Los Alamos, NM.

${ }^{3}$ present address: Yale University, New Haven, Conn.

${ }^{4}$ present address: University of Alberta, Edmonton Alberta 


\section{INTRODUCTION}

\subsection{Hypernuclei}

A nucleus which contains a $\Lambda$ (lambda) hyperon in addition to protons and neutrons is a lambda hypernucleus. Since the bound $\Lambda$ has quantum numbers which distinguishes it from the rest of the nucleons in the hypernuclear system, it is not Pauli excluded from filled nucleon orbitals and can penetrate deep inside the nucleus.

Hypernuclei can be formed by the strong strangeness exchange reaction

$$
\mathrm{K}^{-}+{ }^{\mathrm{A}} \mathrm{Z} \longrightarrow \pi^{-}+{ }_{\Lambda}^{\mathrm{A}} \mathrm{Z}
$$

(see figure 1.1). A $\mathrm{K}^{-}$beam of momentum $750 \mathrm{MeV} / \mathrm{c}$ was incident on ${ }^{4} \mathrm{He}$ and ${ }^{6} \mathrm{Li}$ targets for Brookhaven National Laboratory Experiment 788 at the Alternating Gradient Synchrotron facility[1].

The study of hypernuclei can advance our understanding of details of nuclear structure and provide insight into hyperon-nucleon interactions which are inaccessible in unbound systems. Various measurements of hypernuclei provide the means of exploring nuclear properties such as[3]: the nuclear spin-orbit force, short range correlations, relativistic aspects of many body dynamics, the role of flavor symmetry and the chiral limit, extended models of the strong interaction, weak interactions in the nuclear medium, and possible modifications of baryon properties in the nuclear environment. Hypernuclear studies may provide insight into the three body $\Lambda N N$ weak interaction, which is predicted[4] to play a crucial role. 


\begin{tabular}{|l|c|c|c|c|}
\hline \multicolumn{5}{|c|}{ Particle Properties } \\
\hline \hline name & symbol & Valence Quarks & mass $(\mathrm{MeV})$ & lifetime \\
\hline lambda & $\Lambda$ & $u d s$ & 1115.63 & $263.1 \pm 2.0 p s$ \\
\hline kaon & $K^{-}$ & $\bar{u} s$ & 493.646 & $12.371 \pm 0.0028 n s$ \\
\hline pion & $\pi^{-}$ & $\bar{u} d$ & 139.56755 & $26.029 \pm 0.0023 n s$ \\
& $\pi^{\circ}$ & $\frac{1}{\sqrt{2}}(\bar{u} u-\bar{d} d)$ & 134.97 & $(8.4 \pm 0.6) \times 10^{-17} s$ \\
\hline proton & $p$ & $u u d$ & 938.27231 & stable \\
\hline neutron & $n$ & $u d d$ & 939.56563 & $889.1 \pm 2.1 s$ \\
\hline
\end{tabular}

Table 1.1 Properties[2] of particles commonly refered to throughout this dissertation.

Exploiting the $\Lambda$ hypernucleus to probe nuclear structure began with Cheston and Primakoff[5] in the early 1950's. Hypernuclei were first observed in 1953 by Danysz and Pniewski[6]. Interest in hypernuclei peaked in the early 1960's with the measurements of hyperfragments produced in emulsions and bubble chambers. The sensitivity limits of these measurement techniques were soon reached by the late 1960's. Interest in hypernuclear physics was revived in the late 1970's with the applications of counter measurement techniques. Some of these early counter experiments include work carried out at Carnegie Mellon University[7, 8].

\subsection{The $\Lambda-N$ Weak Interaction}

A free $\Lambda$ predominantly decays via the two mesonic decays

$$
\left.\begin{array}{l}
\Lambda \longrightarrow p \pi^{-} \\
\Lambda \longrightarrow n \pi^{\circ}
\end{array}\right\} \quad \text { Mesonic \& Decay }
$$

with a final state nucleon momentum of about $100 \mathrm{MeV} / \mathrm{c}$. The branching fraction for the semileptonic $\Lambda$ decay is less than $0.2 \%$ and will be ignored in the subsequent discussion. When the $\Lambda$ is embedded in a nucleus, these mesonic decay modes, characterized by the decay widths $\Gamma_{\pi^{-}}$and $\Gamma_{\pi^{\circ}}$, are suppressed, especially in heavier nuclei. The decay nucleon from the $\Lambda \rightarrow N \pi$ reaction, where $N$ can be $n$ or $p$, within the 


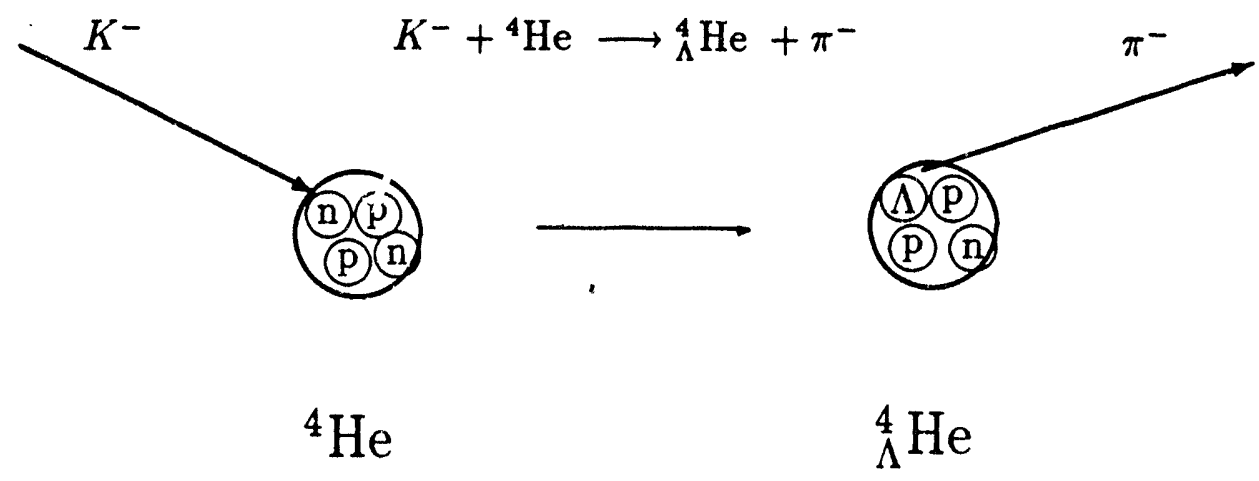

Figure 1.1 A schematic of the $\left(K^{-}, \pi^{-}\right)$reaction on ${ }^{4} \mathrm{He}$ for the formation of ${ }_{\Lambda}^{4} \mathrm{He}$. For this experiment, the ${ }_{\Lambda}^{4} \mathrm{He}$ hypernucleus is formed nearly at rest with a kaon momentum of $750 \mathrm{MeV} / \mathrm{c}$.

daughter nucleus is Pauli blocked from occupying a filled orbital and may not have enough kinetic energy to overcome the binding energy to escape the nuclear core.

A new decay channel is available to the nuclear bound lambda hyperon. The core nucleons can act as catalysts for the subsequent $\Lambda$ decay. This mode is referred to as nucleon stimulated decay,

$$
\left.\begin{array}{l}
\Lambda+p \longrightarrow n+p \\
\Lambda+n \longrightarrow n+n
\end{array}\right\} \quad \text { Nucleon Stimulated } \Lambda \text { Decay }
$$

which is characterized by the decay widths $\Gamma_{p}$ and $\Gamma_{n}$ respectively. The large momentum of the decay nucleons in stimulated decay of $\approx 405 \mathrm{MeV} / \mathrm{c}$ makes this reaction easily identifiable.

\subsubsection{Nuclear Physics Calculations}

There have been numerous theoretical models $[9,10,11,12]$ developed to calculate the decay rates. The measured ratio of neutron to proton stimulated decays $\Gamma_{n} / \Gamma_{p}$ for light hypernuclei is key to understanding the isospin structure of the interaction and differs grtatly from model to model. Unfortınately, even after a decade of experimental research, too little data exist on the ratios $\Gamma_{n} / \Gamma_{p}$, mostly because of inadequate 


\begin{tabular}{|c|c|c|}
\hline \multicolumn{3}{|c|}{ Previous Measurements of ${ }_{\Lambda}^{4} \mathrm{He}$ Decay } \\
\hline \hline Quantity & Value & Reference \\
\hline lifetime & $2.3_{-1.3}^{+2.3} \times 10^{-10} s$ & {$[13]$} \\
\hline$\Gamma_{p}$ & - & no previous data \\
\hline$\Gamma_{n}$ & - & no previous data \\
\hline$\Gamma_{\pi^{-}}$ & - & no previous data \\
\hline$\Gamma_{\pi^{\circ}}$ & - & no previous data \\
\hline$\frac{\Gamma_{n}+\Gamma_{p}}{\Gamma_{r_{-}}}$ & $0.52 \pm 0.13$ & extracted by [14] \\
\hline$\frac{\Gamma_{n}}{\Gamma_{n}+\Gamma_{p}}$ & $0.3 \pm 0.1$ & extracted by [14] \\
\hline
\end{tabular}

Table 1.2 Previous values of quantities measured in E788 for ${ }_{\Lambda}^{4} \mathrm{He}$.

$\Gamma_{n}$ measurements[3]. A review of previous measurements made on ${ }_{\Lambda}^{4} \mathrm{He}$ decays are summarized in table 1.2 .

For one pion exchange calculations the ${ }^{3} \mathrm{~S}_{1} \rightarrow{ }^{3} \mathrm{D}_{1}$ parity-conserving transition dominates. Since the ${ }^{3} \mathrm{D}_{1}$ state must have zero isospin, a two neutron final state is excluded and thus one would expect a neutron stimulated fraction $\Gamma_{n} / \Gamma_{p}<<1$. This may be contrary to Grace's ${ }_{\Lambda}^{12} \mathrm{C}$ measurement $[15,7]$ of $\Gamma_{n} / \Gamma_{p}=1.33_{-0.81}^{+1.12}$. Dubach[10] obtains an enhanced ${ }^{3} \mathrm{~S}_{1},{ }^{3} \mathrm{P}_{1}$ transition when he includes the exchange of $K, K^{*}$, and other heavy mesons. This increases the rate of the neutron stimulated channel, but even with this enhancement his calculations are still below the measured rates. It is also possible that initial $\mathrm{P}$-wave $\Lambda N$ states, not present in the nuclear matter calculations, contribute to the neutron stimulated rates.

\subsubsection{Isospin Structure of $\Delta S=1$ Weak Hamiltonian}

A quark flow diagram for $\Lambda$ decay is shown in figure 1.2. In this picture, the interaction is mediated by the exchange of a $W$ gauge boson between two quarks. The $W$ and $Z$ gauge bosons are the mediators of the weak force in the standard model.

If we could switch off strong interactions, the naive four quark interaction is described by the $\Delta S=1$ Cabibbo form of the weak interaction (for $q^{2}=0$ )

$$
\mathcal{H}_{\Delta S=1}^{C}=\frac{G_{F}}{\sqrt{2}} \cos \theta_{c} \sin \theta_{c}\left(\bar{u} \gamma_{\mu}\left(1-\gamma^{5}\right) s \bar{d} \gamma_{\mu}\left(1-\gamma^{5}\right) u\right)
$$




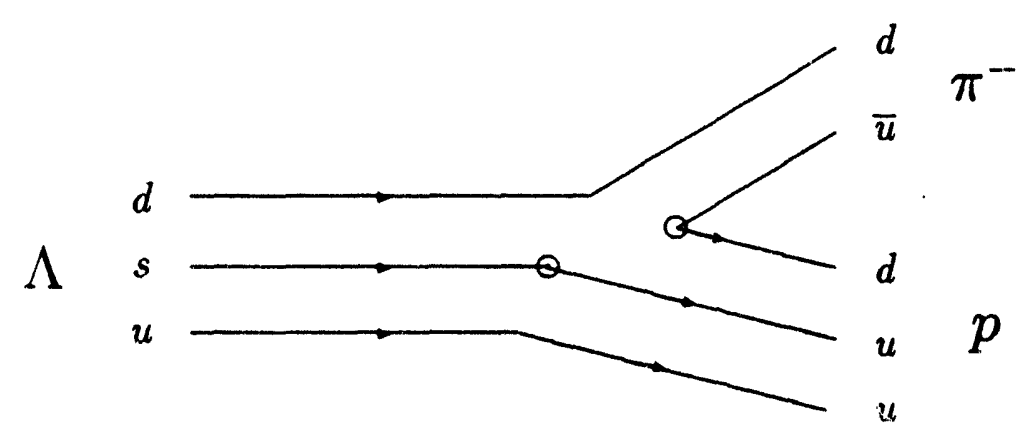

(a)

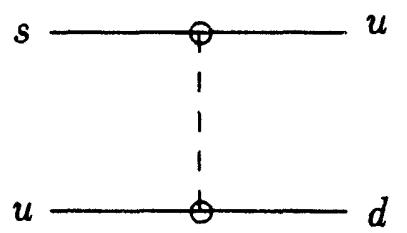

(b)

Figure 1.2 (a) The decay $\Lambda \rightarrow p \pi^{-}$is illustrated in this quark flow diagram. (b) The four fermion weak transition is shown. The dotted line represents $W$ exchange.

This expression corresponds to $W$ boson exchange in the $q^{2}=0$ limit. The factor $\frac{1}{2} \gamma_{\mu}\left(1-\gamma_{5}\right)$ is a chiral projection operator and restricts the interaction to left handed compronents. This factor has a vector minus axial vector character $(V-A)$.

Since the strong interaction seems to overpowers the weak interaction by about $\frac{\alpha_{\text {sor ong }}\left(g^{2}\right)}{G_{F}} \approx 10^{5}$, this may not to be a wonderful approximation, especially when the final states are hadrons. Strong corrections to the weak interaction are expected to play a crucial role. In the presence of QCD corrections equation (1.4) takes the form

$$
\begin{aligned}
& \mathcal{H}_{\Delta S=1}^{g w}=\frac{G_{F}}{\sqrt{2}} \cos \theta_{c} \sin \theta_{c}\left(C_{1} \bar{u} \gamma_{\mu}\left(1-\gamma^{5}\right) s \bar{d} \gamma_{\mu}\left(1-\gamma^{5}\right) u+\right. \\
& \left.C_{2} \bar{d} \gamma^{\mu}\left(1-\gamma^{5}\right) s \bar{u} \gamma_{\mu}\left(1-\gamma^{5}\right) u\right)
\end{aligned}
$$

derived by Gilman and Wise [16]. Their computation includes strong corrections to the interaction Hamiltonian using a leading-log approximation based on one loop diagrams. The Wilson coefficients, $C_{1}$ and $C_{2}$, are determined from the renormalization 
group equations. Assuming a top quark mass of $m_{t}=15 \mathrm{GeV}$ and $\alpha_{\text {strong }}\left(q^{2}\right)=1.00$ they calculated

$$
C_{1}=1.51 \quad C_{2}=-0.856 .
$$

It must be emphasized that Gilman \& Wise take into account only "hard-gluon" exchanges (gluons with energies $\geq 1 \mathrm{GeV}$ ), since only in such cases is $\alpha_{\text {strong }}\left(q^{2}\right)$ small enough to justify perturbation theory. It is much more difficult to properly treat "soft-gluon" effects, which may be pertinent for non-leptonic decays.

There has been confusion about the isospin structure of equation (1.5). A clarification is in order. In the $\mathrm{SU}(2)$ flavor model of the light quarks consists of a doublet, $\left(\begin{array}{l}u \\ d\end{array}\right)$. The $u$ and $d$ quarks each have isospin $\frac{1}{2}$ with $I_{3}$ projections $\frac{1}{2}$ and $-\frac{1}{2}$, respectively. In this model, the $s$ quark has zero isospin. The antiquark equivalents are $\left(\begin{array}{c}\bar{d} \\ -\bar{u}\end{array}\right)$ and $(\bar{s})$. The relation of the quark and antiquark doublet is fundamental to deriving the isospin structure. A more detailed description can be found in appendix A.

The isospin structure of multi-quark operators depend upon their ordering as they appear in the ateraction Hamiltonian. The construction of multi-quark operators is an exercise in angular momentum quantum mechanics. The procedure is similar to the construction of spin states for spin $\frac{1}{2}$ particles using the principle of the lowering operator. A lowering operator $\mathcal{L}$ can be defined such that it transforms the upper component of a doublet into the lower. The rules for lowering quarks and antiquarks are

$$
\mathcal{L} u=d, \quad \mathcal{L} d=0, \quad \mathcal{L} \bar{d}=-\bar{u}, \quad \mathcal{L} \bar{u}=0 .
$$

The construction of $q q$ and $q \bar{q}$ operator pairs is accomplished by first starting with state of maximum $I_{3},\left(I, I_{3}\right)=(1,1)$, and successively applying the lowering operator to it.

\begin{tabular}{lcc}
$\left(I, I_{3}\right)$ & $q q$ & $q \bar{q}$ \\
\hline & & \\
$(1,1)$ & $u u$ & $u \bar{d}$ \\
$(1,0)$ & $\frac{1}{\sqrt{2}}(u d+d u)$ & $\frac{1}{\sqrt{2}}(d \bar{d}-u \bar{u})$ \\
$(1,-1)$ & $d d$ & $-d \bar{u}$ \\
$(0,0)$ & $\frac{-1}{\sqrt{2}}(u d-d u)$ & $\frac{1}{\sqrt{2}}(d \bar{d}+u \bar{u})$
\end{tabular}


To understand the isospin structure of equation (1.5) it is only necessary to examine the doublet quarks $u, d, \bar{u}, \bar{d}$ - the $s$ quark carries 0 isospin and does not contribute to the overall structure. The $s$ quark appears in both terms of equation (1.5). Thus, we need only be concerned with three quark operators. The $\bar{q} \bar{q} q$ isospin decomposition is given by

$$
\begin{array}{cc}
\left(I, I_{3}\right) & \bar{q} \bar{q} q \\
\left(\frac{3}{2}, \frac{3}{2}\right) & \bar{d} \bar{d} u \\
\left(\frac{3}{2}, \frac{1}{2}\right) & \frac{1}{\sqrt{3}}(\bar{d} \bar{d} d-\bar{u} \bar{d} u-\bar{d} \bar{u} u) \\
\left(\frac{3}{2}, \frac{-1}{2}\right) & \frac{1}{\sqrt{3}}(\bar{u} \bar{u} u-\bar{d} \bar{u} d-\bar{u} \bar{d} d) \\
\left(\frac{3}{2}, \frac{-3}{2}\right) & \bar{u} \bar{u} d \\
& \\
\left(\frac{1}{2}, \frac{1}{2}\right) & \frac{1}{\sqrt{6}}(2 \bar{d} \bar{d} d+\bar{u} \bar{d} u+\bar{d} \bar{u} u) \\
\left(\frac{1}{2}, \frac{-1}{2}\right) & \frac{1}{\sqrt{6}}(2 \bar{u} \bar{u} u+\bar{d} \bar{u} d+\bar{u} \bar{d} d) \\
\left(\frac{1}{2}, \frac{1}{2}\right)^{\prime} & \frac{1}{\sqrt{2}}(\bar{u} \bar{d} u-\bar{d} \bar{u} u) \\
\left(\frac{1}{2}, \frac{-1}{2}\right)^{\prime} & \frac{1}{\sqrt{2}}(\bar{d} \bar{u} d-\bar{u} \bar{d} d) .
\end{array}
$$

The $\left(\frac{1}{2}, \pm \frac{1}{2}\right)$ and $\left(\frac{1}{2}, \pm \frac{1}{2}\right)^{\prime}$ components are constructed by requiring orthogonality with the $\left(\frac{3}{2}, \pm \frac{1}{2}\right)$ components and with each other. These relations can be inverted to solve for the $\left(I, \frac{1}{2}\right)$ operators of interest.

$$
\begin{aligned}
& \bar{d} \bar{d} d=\frac{1}{\sqrt{3}}\left(\frac{3}{2}, \frac{1}{2}\right)+\sqrt{\frac{2}{3}}\left(\frac{1}{2}, \frac{1}{2}\right)^{\prime} \\
& \bar{u} \bar{d} u=\frac{-1}{\sqrt{3}}\left(\frac{3}{2}, \frac{1}{2}\right)+\frac{1}{\sqrt{6}}\left(\frac{1}{2}, \frac{1}{2}\right)^{\prime}+\frac{1}{\sqrt{2}}\left(\frac{1}{2}, \frac{1}{2}\right) \\
& \bar{d} \bar{u} u=\frac{-1}{\sqrt{3}}\left(\frac{3}{2}, \frac{1}{2}\right)+\frac{1}{\sqrt{6}}\left(\frac{1}{2}, \frac{1}{2}\right)^{\prime}-\frac{1}{\sqrt{2}}\left(\frac{1}{2}, \frac{1}{2}\right)
\end{aligned}
$$

Now we can relate this result to the isospin structure of $\mathcal{H}_{\Delta S=1}^{g w}$. Substituting the above isospin expansion states into equation (1.5) yields

$$
\begin{aligned}
& C_{1} \bar{u} \bar{d} u+C_{2} \bar{d} \bar{u} u= \\
& -\sqrt{\frac{1}{3}}\left(C_{1}+C_{2}\right)\left(\frac{3}{2}, \frac{1}{2}\right)+\sqrt{\frac{1}{6}}\left(C_{1}+C_{2}\right)\left(\frac{1}{2}, \frac{1}{2}\right)^{\prime}+\sqrt{\frac{1}{2}}\left(C_{1}-C_{2}\right)\left(\frac{1}{2}, \frac{1}{2}\right) .
\end{aligned}
$$


A ratio can be constructed giving the relative $\left(\frac{3}{2}, \pm \frac{1}{2}\right)$ strength to the $\left(\frac{1}{2}, \pm \frac{1}{2}\right)^{(\prime)}$ strengths

$$
R=\frac{\sqrt{\frac{1}{3}}\left(C_{1}+C_{2}\right)}{\sqrt{\frac{1}{6}}\left(C_{1}+C_{2}\right)+\sqrt{\frac{1}{2}}\left(C_{1}-C_{2}\right)}
$$

If $C_{2}=-C_{1}$ then we have a pure $\left(\frac{1}{2}, \frac{1}{2}\right)$ operator. On the contrary, when $C_{2}=C_{1}$ we do not have $a\left(\frac{3}{2}, \frac{1}{2}\right)$ operator as alluded to in the paper by Heddle \& Kisslinger[12] since the $\left(\frac{3}{2}, \frac{1}{2}\right)$ operator has a $\bar{d} \bar{d} d$ term not accounted for in their interaction Hamiltonian.

To include the values of $C_{1}$ and $C_{2}$ given by Gilman \& Wise (equation " 5 )) yields $R=0.19$ which is far from the empirical $\Delta I=\frac{1}{2}$ value of $R=0.05$. it is interesting to note that for the oversimplified Cabibbo Hamiltonian (equation (1.4)) has $R=0.52$ which indicates that the Gilman \& Wise renormalization calculation does not necessarily enhance the $\Delta I=\frac{1}{2}$ rule as much as the advertised factor of 4 in amplitude.

\subsection{The $\Delta I=\frac{1}{2}$ Rule}

It has been pointed out $[3,17]$ that further measurements of light hypernuclear systems will provide insight into the form of the effective Hamiltonian governing weak interactions in the presence of hadronic fields. One goal is a fundamental understanding of the empirical $\Delta I=\frac{1}{2}$ rule in weak interactions which has been observed in $\Delta S=1$ mesonic decays. This rule is an observation of the strong dominance of $\Delta I=\frac{1}{2}$ amplitudes over $\Delta I=\frac{3}{2}$ amplitudes for mesonic strangeness-changing weak decays.

\subsubsection{Free $\Lambda$ Decay}

The $\Delta I=\frac{1}{2}$ effect is illustrated for the case of free $\Lambda$ decays (equation (1.2)). The isospin transition amplitude is expressed as

$$
\left\langle N \pi\left|\mathcal{H}^{I}\right| \Lambda\right\rangle=\left\langle N \pi\left|a_{1}\left(\frac{1}{2}, \frac{1}{2}\right)+a_{3}\left(\frac{3}{2}, \frac{1}{2}\right)\right| \Lambda\right\rangle
$$


where $a_{i}$ are the strengths of the $\Delta I=\frac{i}{2}$ amplitudes. For the two decay modes, the isospin transition amplitudes caun be decomposed into

$$
\begin{aligned}
\left\langle n \pi^{\circ}\left|a_{1}\left(\frac{1}{2}, \frac{1}{2}\right)+a_{3}\left(\frac{3}{2}, \frac{1}{2}\right)\right| \Lambda\right\rangle & =\sqrt{\frac{1}{3}} a_{1} A_{1}+\sqrt{\frac{2}{3}} a_{3} A_{3} \\
\left\langle p \pi^{-}\left|a_{1}\left(\frac{1}{2}, \frac{1}{2}\right)+a_{3}\left(\frac{3}{2}, \frac{1}{2}\right)\right| \Lambda\right\rangle & =-\sqrt{\frac{2}{3}} a_{1} A_{1}+\sqrt{\frac{1}{3}} a_{3} A_{3}
\end{aligned}
$$

where

$$
A_{1}=<f\left\|\left(\frac{1}{2}\right)\right\| i>, \quad A_{3}=<f\left\|\left(\frac{3}{2}\right)\right\| i>.
$$

This decomposition is achieved by substituting the $\left\langle I I_{z}\right|$ bra's $\left\langle\frac{1}{2} \frac{1}{2}\right|,\left\langle\frac{1}{2}-\frac{1}{2}\right|,\langle 10|$, and $\langle 1-1|$ for $p, n, \pi^{\circ}$, and $\pi^{-}$, respectively. If one ignores the $\Delta I=\frac{3}{2}$ amplitudes (let $a_{3}=0$ ) the ratio of the transition rates is

$$
\frac{\Gamma\left(\Lambda \rightarrow p \pi^{-}\right)}{\Gamma\left(\Lambda \rightarrow n \pi^{\circ}\right)} \stackrel{\Delta I=\frac{1}{2}}{=}\left|\frac{\left\langle p \pi^{-}\left|\mathcal{H}^{I}\right| \Lambda\right\rangle}{\left\langle n \pi^{\circ}\left|\mathcal{H}^{I}\right| \Lambda\right\rangle}\right|^{2}=\frac{2}{1}
$$

which agrees very well with the measured branching fractions[2]

$$
\begin{array}{lr}
\Lambda \longrightarrow p \pi^{-} & 64.1 \% \\
\Lambda \longrightarrow n \pi^{\circ} & 35.7 \% .
\end{array}
$$

The $\Delta I=\frac{1}{2}$ rule seems to apply well to the decay of kaons and most strange baryons; however, it does not seem to work well for $\Omega^{-}$decays[18].

\subsubsection{The $\Delta I=\frac{1}{2}$ Rule and Hypernuclear Decay}

Measurements of the proton and neutron stimulated decay rates for a few hypernuclei can determine the relative strength of the $\Delta I=\frac{1}{2}$ and $\Delta I=\frac{3}{2}$ amplitudes for $I=1$ final states. The initial state for the $\Lambda N$ pair in the light hypernuclear systems ${ }_{\Lambda}^{3} \mathrm{H}$, ${ }_{\Lambda}^{4} \mathrm{H},{ }_{\Lambda}^{4} \mathrm{He}$, and ${ }_{\Lambda}^{5} \mathrm{He}$ each have different spin and isospin configurations. The isospin dependence can be extracted from combinations of various measurements of these systems.

To illustrate this, consider an arbitrary interaction Hamiltonian $\mathcal{H}^{I}$ composed of $\left(\frac{3}{2}, \frac{1}{2}\right)$ and $\left(\frac{1}{2}, \frac{1}{2}\right)$ transition operators

$$
\mathcal{H}^{I}=a_{1}\left(\frac{1}{2}, \frac{1}{2}\right)+a_{3}\left(\frac{3}{2}, \frac{1}{2}\right)
$$


where $a_{1}$ and $a_{3}$ are to be determined. The transition amplitudes for neutron and proton stimulated decay restricted to $I=1$ final states is then expressed as

$$
\begin{aligned}
\left\langle n p\left|\mathcal{H}^{I}\right| \Lambda p\right\rangle & =a_{1}\left\langle 10\left|\left(\frac{1}{2}, \frac{1}{2}\right)\right| \frac{1}{2} \frac{1}{2}\right\rangle+a_{3}\left(10\left|\left(\frac{3}{2}, \frac{1}{2}\right)\right| \frac{1}{2} \frac{1}{2}\right\rangle \\
\left\langle n n\left|\mathcal{H}^{I}\right| \Lambda n\right\rangle & =a_{1}\left\langle 1-1\left|\left(\frac{1}{2}, \frac{1}{2}\right)\right| \frac{1}{2}-\frac{1}{2}\right\rangle+a_{3}\left\langle 1-1\left|\left(\frac{3}{2}, \frac{1}{2}\right)\right| \frac{1}{2}-\frac{1}{2}\right\rangle .
\end{aligned}
$$

These matrix elements are reduced further using the Wigner-Eckart theorem which prescribes how the $I_{3}$ dependence is removed. The expectation of a tensor operator $\left(I, \frac{1}{2}\right)$ is related to the reduced matrix element by[3]

$$
\left\langle I_{f} I_{f_{z}}\left|\left(I, \frac{1}{2}\right)\right| I_{i} I_{i_{z}}\right\rangle=\left(\begin{array}{ccc}
I_{f} & I & I_{i} \\
-I_{f_{z}} & -\frac{1}{2} & I_{i_{z}}
\end{array}\right)(-1)^{I-I_{f_{z}}}\left\langle I_{f}\|(I)\| I_{i}\right\rangle
$$

where the $2 \times 3$ matrix is the Wigner 3 -j symbol. The four matrix elements we are interested in are reduced to

$$
\begin{aligned}
\left\langle 1-1\left|\left(\frac{1}{2}, \frac{1}{2}\right)\right| \frac{1}{2}-\frac{1}{2}\right\rangle & =-\frac{1}{\sqrt{3}}\left\langle 1\left\|\left(\frac{1}{2}\right)\right\| \frac{1}{2}\right\rangle \\
\left\langle 10\left|\left(\frac{1}{2}, \frac{1}{2}\right)\right| \frac{1}{2} \frac{1}{2}\right\rangle & =-\frac{1}{\sqrt{6}}\left\langle 1\left\|\left(\frac{1}{2}\right)\right\| \frac{1}{2}\right\rangle \\
\left\langle 1-1\left|\left(\frac{3}{2}, \frac{1}{2}\right)\right| \frac{1}{2}-\frac{1}{2}\right\rangle & =\frac{1}{\sqrt{12}}\left\langle 1\left\|\left(\frac{3}{2}\right)\right\| \frac{1}{2}\right\rangle \\
\left\langle 10\left|\left(\frac{3}{2}, \frac{1}{2}\right)\right| \frac{1}{2} \frac{1}{2}\right\rangle & =-\frac{1}{\sqrt{6}}\left\langle 1\left\|\left(\frac{3}{2}\right)\right\| \frac{1}{2}\right\rangle .
\end{aligned}
$$

We can now construct a simple expression which relates the ratio of the isospin amplitudes to the stimulated decay amplitudes

$$
\left.\epsilon \equiv \frac{\langle\Lambda n \rightarrow n n\rangle}{\langle\Lambda p \rightarrow n p\rangle}\right|_{I_{f}=1}=\frac{1}{\sqrt{2}}\left(\frac{r-2}{r+1}\right)
$$

wbere

$$
r=\frac{a_{3}}{a_{1}} \frac{\left\langle 1\left\|\left(\frac{3}{2}\right)\right\| \frac{1}{2}\right\rangle}{\left\langle 1\left\|\left(\frac{1}{2}\right)\right\| \frac{1}{2}\right\rangle}
$$

If $r=0$ we obtain the $\Delta I=\frac{1}{2}$ prediction that the neutron rate is twice that of the proton rate, $\epsilon^{2}=2$. To reiterate, this prediction only applies to $I=1$ final states. 


\section{Isospin Selection in Hypernuclear Decay}

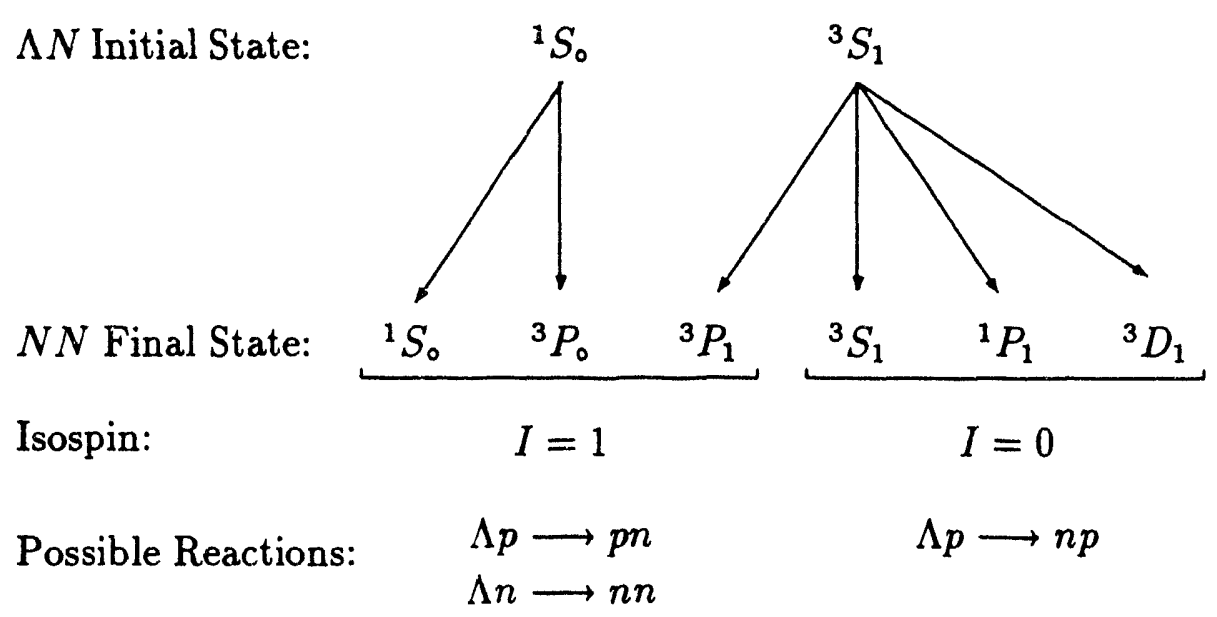

Figure 1.3 For a given $\Lambda N$ initial state, the selection of possible final states are based upon parity and conservation of angular momentum. $I=0$ final states are only available to proton stimulated decay, while $I=1$ final states permit both proton and neutron stimulated decay.

\section{Rate Extraction from Data}

Figure 1.3 illustrates the available transitions for the singlet and triplet $\Lambda N$ state in hypernuclear systems. It is reasonable to assume the $\Lambda$ and $N$ are in an $S$ state.

An extensive treatment of lambda hypernuclear weak decay modes has been presented by Block and Dalitz[9]. In their study, the stimulated $\Lambda$ decay is treated as incoherent. This procedure neglects final state interactions for the two energetic outgoing nucleons and interference effects arising from antisymmetrization of the final state.

In their model, they define the partial capture rate $R_{N S}$ from spin state $S$ of nucleon $N$ where $N$ could be $p$ or $n$. For example, starting from a spin singlet $S=0$ state with a proton stimulated transition we have $R_{p 0}=\mathcal{R}\left({ }^{1} S_{0} \rightarrow{ }^{1} S_{0}\right)+\mathcal{R}\left({ }^{1} S_{0} \rightarrow\right.$ ${ }^{3} P_{0}$ ) (see figure 1.3). The square of the ratio of amplitudes of equation (1.22) is actually $R_{n 0} / R_{p 0}$ since the $I=1$ final state corresponds to the $S=0$ spin state.

The non-mesonic decay rate for a hypernucleus ${ }_{A}^{A} \mathrm{Z}$ is given in this model with a 


\begin{tabular}{|c|c|c|}
\hline \multicolumn{3}{|c|}{ Allowed Initial States } \\
\hline \hline Hypernucleus & $\Lambda n$ & $\Lambda p$ \\
\hline${ }^{12} \mathrm{C}$ & ${ }^{1} S_{\circ}{ }^{3} S_{1} P$ & ${ }^{1} S_{\circ}{ }^{3} S_{1} P$ \\
\hline$\star{ }_{\Lambda}^{5} \mathrm{He}$ & ${ }^{1} S_{\circ}{ }^{3} S_{1}$ & ${ }^{1} S_{\circ}{ }^{3} S_{1}$ \\
\hline$\star{ }_{\Lambda}^{4} \mathrm{He}$ & ${ }^{1} S_{\circ}$ & ${ }^{1} S_{\circ}{ }^{3} S_{1}$ \\
\hline${ }_{\Lambda}^{4} \mathrm{H}$ & ${ }^{1} S_{\circ}{ }^{3} S_{1}$ & ${ }^{1} S_{\circ}$ \\
\hline
\end{tabular}

Table 1.3 The initial states for $\Lambda N$ configurations are based upon the spin of the hypernucleus. The $\star$ iridicates measurements made in the recent $B N L$ experiment E788

local $\Lambda N$ interaction by

$$
\Gamma_{n m}\left({ }_{\Lambda}^{A} \mathrm{Z}\right)=\rho_{A} \overline{\mathcal{R}}\left({ }_{\Lambda}^{A} \mathrm{Z}\right)
$$

where $\overline{\mathcal{R}}\left({ }_{\Lambda}^{A} \mathrm{Z}\right)$ is the spin-isospin average of the $R_{N S}$ for this hypernucleus, and

$$
\rho_{A}==(A-1) \int u_{\Lambda}^{2}(\vec{r}) \rho_{N}(\vec{r}) d \vec{r}
$$

is the mean nucleon density at the $\Lambda$ position with $u_{\Lambda}$ being the $\Lambda$ wave function and $\rho_{N}$ the nucleon density.

The non-mesonic decay rates for various light hypernuclei can then be tabulated[3] in accordance to equation (1.23):

$$
\begin{aligned}
\Gamma_{n m}\left({ }_{\Lambda}^{5} \mathrm{He}\right) & =\rho_{5} \bar{R}\left({ }_{\Lambda}^{5} \mathrm{He}\right)=\frac{1}{8} \rho_{5}\left(3 R_{p 1}+R_{p 0}+3 R_{n 1}+R_{n 0}\right) \\
\Gamma_{n m}\left({ }_{\Lambda}^{4} \mathrm{He}\right) & =\rho_{4} \bar{R}\left({ }_{\Lambda}^{4} \mathrm{He}\right)=\frac{1}{6} \rho_{4}\left(3 R_{p 1}+R_{p 0}+2 R_{n 0}\right) \\
\Gamma_{n m}\left({ }_{\Lambda}^{4} \mathrm{H}\right) & =\rho_{4} \bar{R}\left({ }_{\Lambda}^{4} \mathrm{H}\right)=\frac{1}{6} \rho_{4}\left(2 R_{p 0}+3 R_{n 1}+R_{n 0}\right) .
\end{aligned}
$$

These three measured decay rates can be inverted to solve for the ratio of interest, $R_{n 0} / R_{p 0}$, of equation (1.22). Dover[17] carried out this linear algebra task with the aid of the definitions

$$
\gamma_{n m}=\frac{\Gamma_{n m}\left({ }_{\Lambda}^{4} \mathrm{He}\right)}{\Gamma_{n m}\left({ }_{\Lambda}^{4} \mathrm{H}\right)}, \quad \gamma_{n p}^{4}=\frac{\Gamma_{n}\left({ }_{\Lambda}^{4} \mathrm{He}\right)}{\Gamma_{p}\left({ }_{\Lambda}^{4} \mathrm{He}\right)}, \quad \gamma_{n p}^{5}=\frac{\Gamma_{n}\left({ }_{\Lambda}^{5} \mathrm{He}\right)}{\Gamma_{p}\left({ }_{\Lambda}^{5} \mathrm{He}\right)} .
$$

The rates given in equation (1.25) can be folded with the above definitions to relate the ratio of the stimulatied decay rate to the various measurements

$$
\epsilon^{2}=\frac{R_{n 0}}{R_{p 0}}=\frac{\gamma_{n m} \gamma_{n p}^{4}}{1+\gamma_{n p}^{4}-\gamma_{n m} \gamma_{n p}^{5}}
$$


Since measurements on ${ }_{\Lambda}^{4} \mathrm{H}$ are sparse, the expression for $\gamma_{n m}$ can be expanded without approximation to

$$
\gamma_{n m}=\frac{\tau\left({ }_{\Lambda}^{4} \mathrm{H}\right)}{\tau\left({ }_{\Lambda}^{4} \mathrm{He}\right)} \frac{Q^{-}\left({ }_{\Lambda}^{4} \mathrm{He}\right)}{Q^{-}\left({ }_{\Lambda}^{4} \mathrm{H}\right)} \frac{\left(1+R_{\circ}+Q^{-}\right){ }_{\Lambda_{\Lambda} \mathrm{H}}}{\left(1+R_{\circ}+Q^{-}\right){ }_{\Lambda} \mathrm{He}}
$$

where

$$
\begin{gathered}
Q^{-}=\frac{\Gamma_{n m}}{\Gamma_{\pi^{-}}} \\
R_{\mathrm{o}}=\frac{\Gamma_{\pi^{\circ}}}{\Gamma_{\pi^{-}}} .
\end{gathered}
$$

An analysis attempt of existing data has been made by Schumacher[19]. A discussion of the current value of $\epsilon^{2}$ based on this analysis is found in chapter 5 .

\subsection{E788 ${ }_{\Lambda}^{4} \mathrm{He}$ Measurements}

As pointed out earlier, there is very little known about the decay of ${ }_{\Lambda}^{4} \mathrm{He}$ (table 1.2). The measurement goals of the ${ }_{\Lambda}^{4} \mathrm{He}$ experiment are

- determine the mesonic decay rate $\Gamma_{\pi^{-}}$

- determine the proton stimulated decay rate $\Gamma_{p}$

- determine the neutron stimulated decay rate $\Gamma_{n}$.

- determine the total lifetime

$$
\tau=\frac{1}{\Gamma_{p}+\Gamma_{n}+\Gamma_{\pi-}+\Gamma_{\pi^{\circ}}}
$$

- determine the kinetic energy distributions for the various decay products

The following chapter describes the experimental apparatus used in the measurement of the decay of ${ }_{\Lambda}^{4} \mathrm{He}$. Chapter 3 provides a description of the calibration and normalization procedures used to obtain the final measurements described in chapter 4 . Conclusions and discussion of the results are provided in chapter 5 . 
INTRODUCTION

-

-

-

| 


\section{APPARATUS}

\subsection{Experiment Beam Transport}

The ${ }_{\Lambda}^{4} \mathrm{He}$ decay experiment was executed at Brookhaven National Laboratory during the winter of 1990. Protons are accelerated to an energy of $28 \mathrm{GeV}$ around a 1.2 kilometer circumference Alternating Gradient Synchrotron (AGS) with a machine cycle time of 3.2 seconds. Slow extraction of the accelerated protons occurs 2 seconds into the cycle time and provides a total of $12 \times 10^{12}$ protons per spill. The protons are shared and distributed to several experiments throughout the AGS experimental hall.

One such destination for the accelerated protons is the primary target for the C6 beamline. It is this beamline which is employed by the hypernuclear lifetime experiment. The primary target consists of a piece of platinum of length about $2 \mathrm{~cm}$.

The proton interactions with the platinum primary target result in a flood of secondary particles. A secondary beam transport system provides a mechanism for the extraction of a specific species of particles within a prescribed momentum range. The $\mathrm{C} 6$ secondary beamline, which feeds the hypernuclear lifetime experiment, can be decomposed into (1) momentum/charge selection, (2) mass selection, (3) target incident momentum analysis, and (4) target scattered momentum analysis sections (figure 2.1).

Negatively charged particles are swept from the primary target at an angle of $15^{\circ}$ 


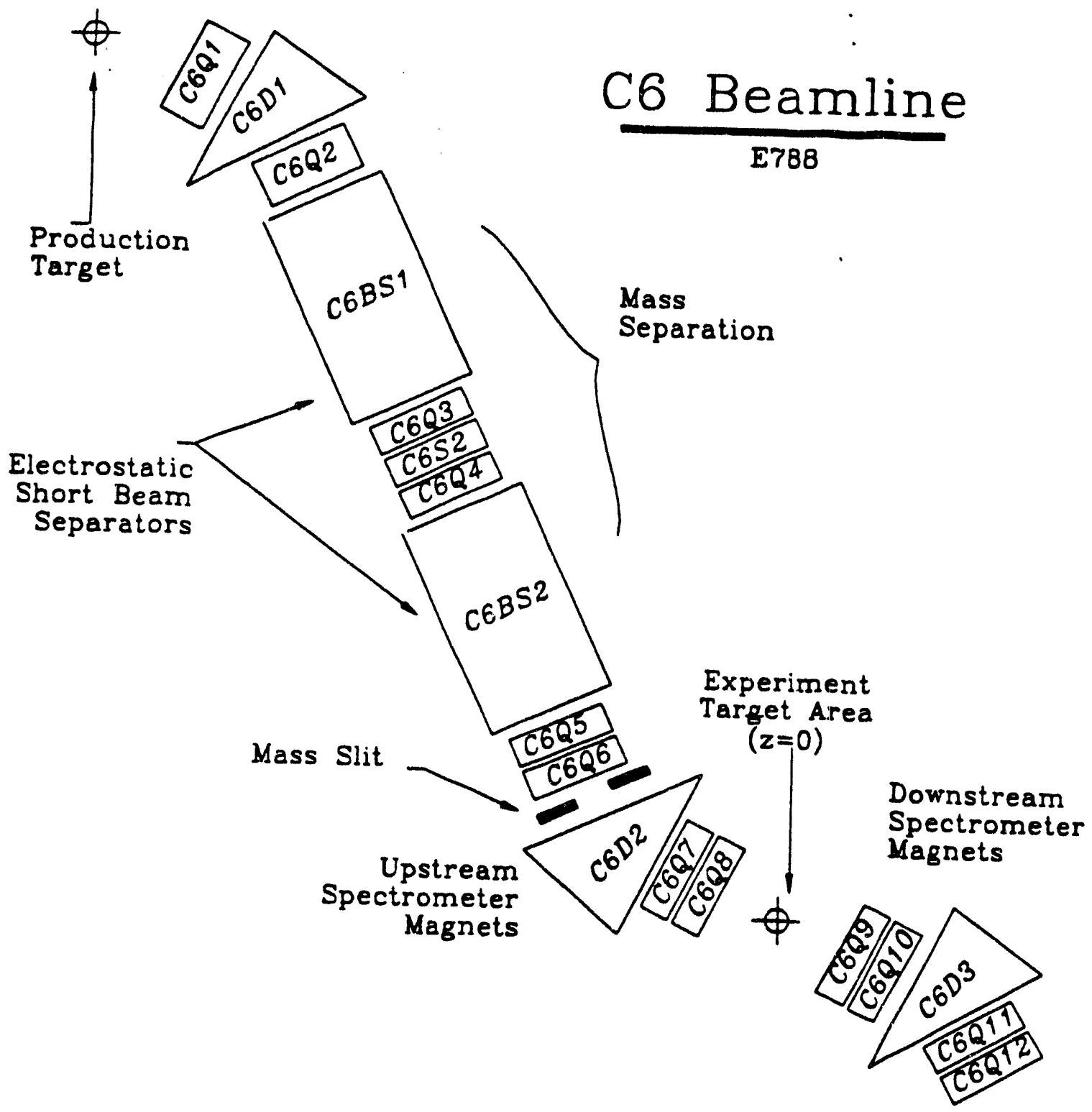

Figure 2.1 Schematic of secondary beamline C6 used in experiment E788. The beamline can be sectioned into four parts: (1) momentum/charge selection (C6Q1 to C6Q2), (2) mass selection (C6BS1 to C6BS2), (3) target incident momentuim analysis (C6Q5 to C6Q8), and (4) target scattered momentum analysis (C6Q9 to C6Q12). The $z$ direction is defined along the 
with a central momentum of $750 \mathrm{MeV} / \mathrm{c} \pm 2 \%$ by a quadrapole-dipole-quadrapole ( C6Q1 - C6D1 - C6Q2 ) magnet configuration (see figure 2.1). The dipole magnet is used for beam particle momentum selection while the higher order magnets provide focusing.

The beamline provides a high intensity source of low energy kaons to the experimentalist $1100 \mathrm{~cm}$ down from the production target. What makes this a formidable task is the relatively short lifetime[2] of the $K^{-}, \tau=12.4 n s$. Also, the $\pi^{-}$'s and $\mu^{-'}$ 's from the kaon decay,

$$
\begin{aligned}
K^{-} \longrightarrow \pi^{0}+\pi^{-} & 21.2 \% \\
\longrightarrow \mu+\bar{\nu}_{\mu} & 63.5 \%
\end{aligned}
$$

contribute to the background contaminants of the beam composition. For a kaon of momentum $750 \mathrm{Mev} / \mathrm{c}$, the mean decay length is $\lambda=563 \mathrm{~cm}$. From this, the fraction of the $K^{-}$flux remaining at the experiment area $1100 \mathrm{~cm}$ from the primary target is $e^{-x / 563 \mathrm{~cm}} \approx 14.2 \%$. That is, 86 out of every 100 kaons produced in the primary target will decay by the time they get to the target area.

The contaminant content is diminished by the method of velocity separation. Mass selection is achieved by a pair of electrostatic beam separators which consist of capacitor plates above and below the beam along with a dipole magnet which provides a horizontal magnetic field. A set of focusing magnet elements inserted between them ( C6BS1 - C6Q3 - C6S2 - C6Q4 - C6BS2 ). The force applied to a charged particle of momentum $p$ traversing perpendicular magnetic and electric fields is given by

$$
\mathbf{F}=e(\mathbf{E}+\mathbf{v} \times \mathbf{B})
$$

where $\mathbf{v}$ is the velocity. The electric $\mathbf{E}$ and magnetic $\mathbf{B}$ fields were chosen so that zero net force is exerted upon a $750 \mathrm{MeV} \mathrm{K}^{-}$. Pions are diverted from the main beam axis and are stopped in a high density aperture (mass-slit).

\subsection{1 $K^{-}$and $\pi^{-}$Spectrometers}

The momentum of a charged particle is determined from its trajectory deviation in a dipole magnetic field. The detectors used to measure the trajectory are discussed in section 2.2.1. The magnet configuration $\mathrm{C} 6 \mathrm{Q} 5-\mathrm{C} 6 \mathrm{Q} 6-\mathrm{C} 6 \mathrm{D} 2-\mathrm{C} 6 \mathrm{Q} 7-\mathrm{C} 6 \mathrm{Q} 8$ provides quadrapole focusing before and after the dipole $\mathrm{C} 6 \mathrm{D} 2$. To determine the excitation 


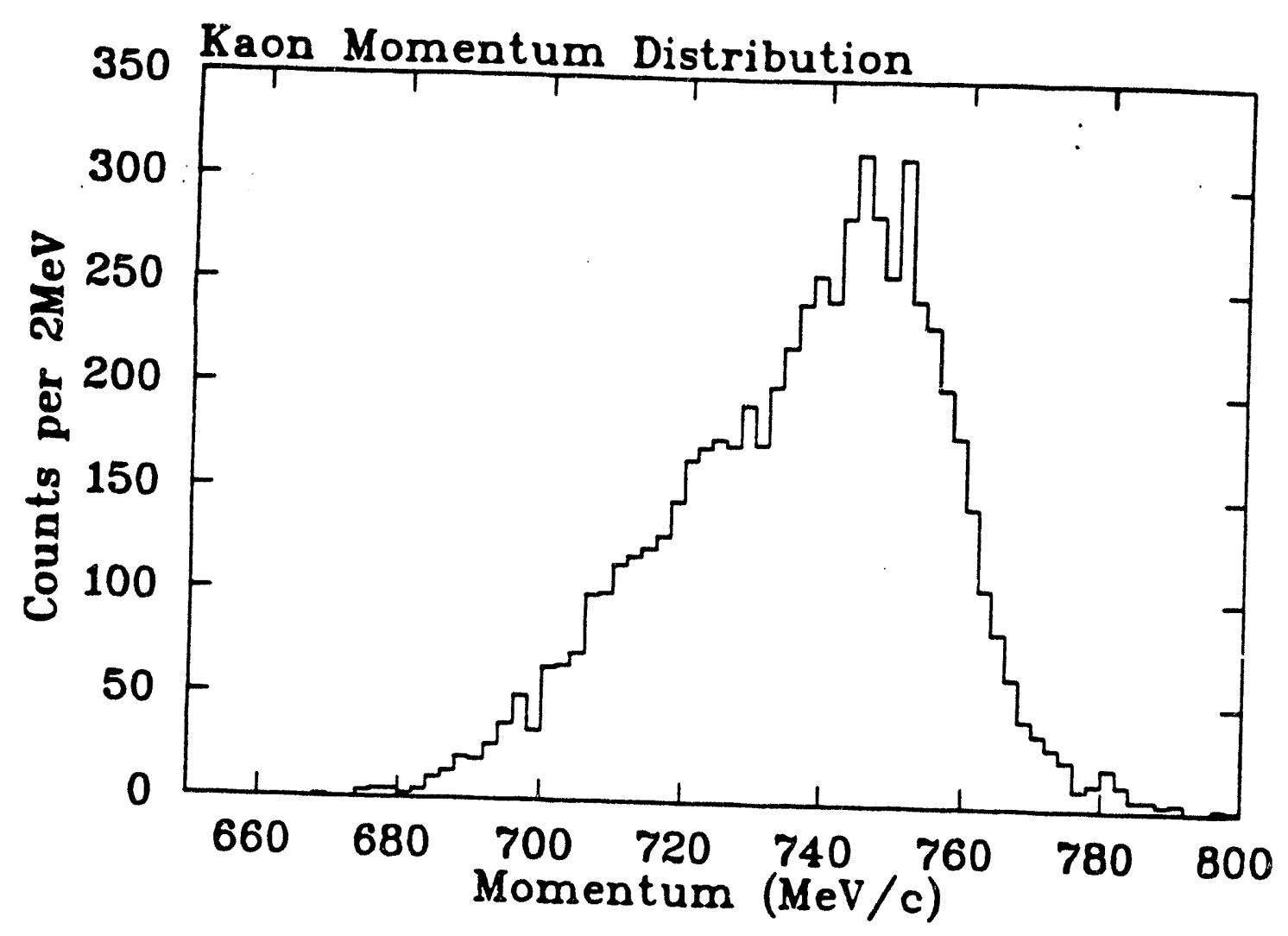

Figure 2.2 Kaon momentum distribution as measured in upstream kaon spectrometer. The kaon identity requirement is established from a time of flight (velocity) measurement.

energy, we use spectrometers to measure the momentum of both incident and scattered particles. The dependence of the excitation energy resolution upon the target incident's momentum resolution is roughly $\sigma_{E E} \approx \beta_{K} \sigma_{P K}$. The momentum distribution for kaons at the target is shown in figure 2.2. Figure 2.3 illustrates beam $x$ and $y$ distributions at $z=0$.

The pion spectrometer has focusing elements before and after the analyzing dipole: C6Q9-C6Q10-C6D3-C6Q11-C6Q12. The entire down-stream spectrometer is capable of rotating about a pivot point at $z=0$. For the ${ }_{A}^{4} \mathrm{He}$ experiment, the spectrometer is fixed at zero degrees. The momentum distribution for pions as measured by the downstream spectrometer is shown in figure 2.4 . 

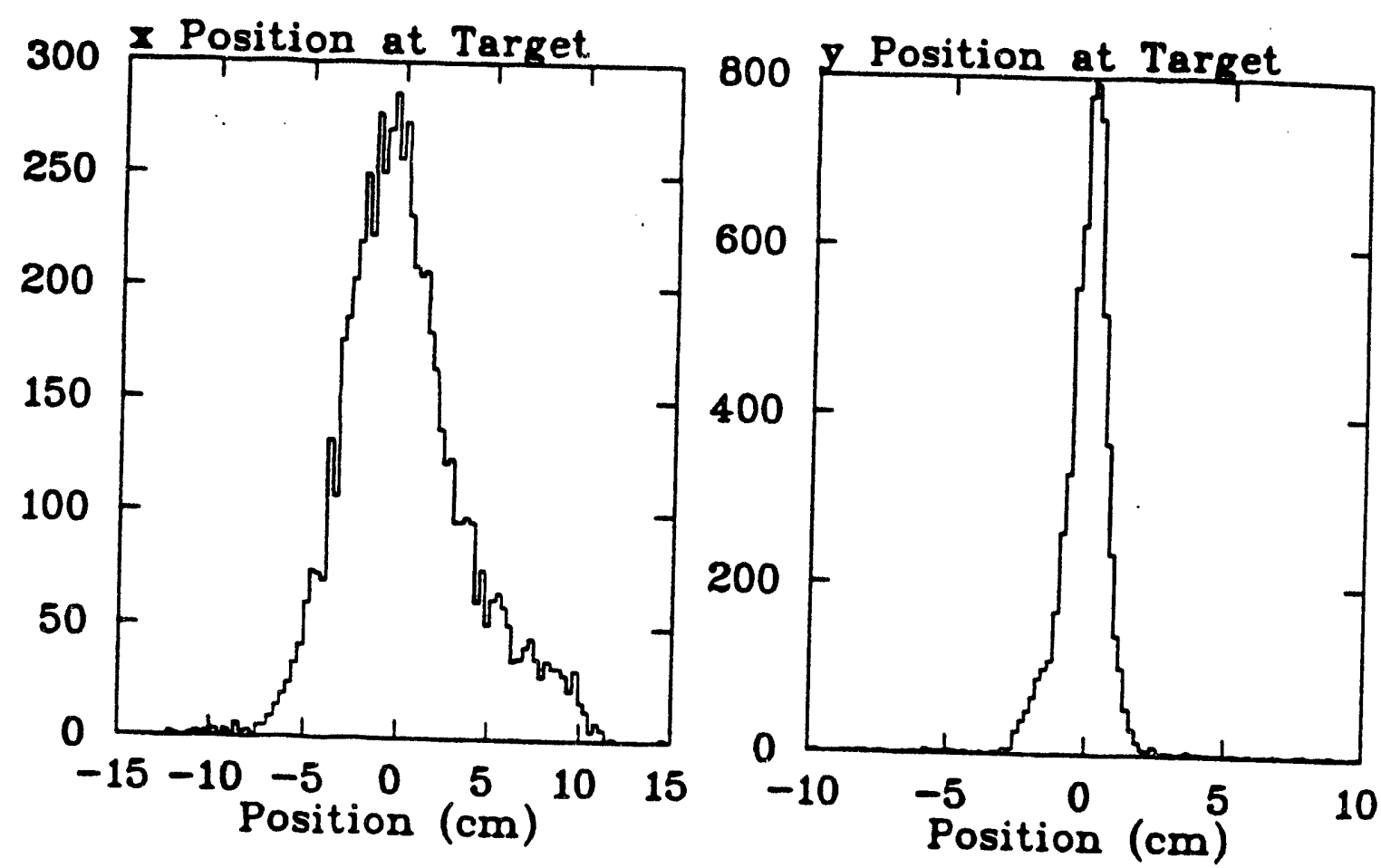

Figure 2.3 $x$ and $y$ beam distributions at target $z=0$ for kaons. The $z$ direction is defined along the beam axis, $y$ is upward, and $x$ to the beam left.

\subsection{Beamline Instrumentation}

\subsubsection{In-Beam Tracking}

There is a total of fourteen drift chambers used in this experiment. The designated name, $z$ coordinate, and orientation are tabulated in table 2.1 (the $z$ direction is defined along the beam axis). A schematic drawing illustrating the chamber positions along the upstream and downstream spectrometers is given in figure 2.5. The drift chambers were constructed to deal with the high rates present in the beam. All of the drift chambers measure one coordinate with two planes of sense wires. The two planes are necessary to determine on which side of a given signal wire the charged particle track crossed the wire plane. The drift cell separation is $0.508 \mathrm{~cm}$. The separation between planes is $0.43 \mathrm{~cm}$. 


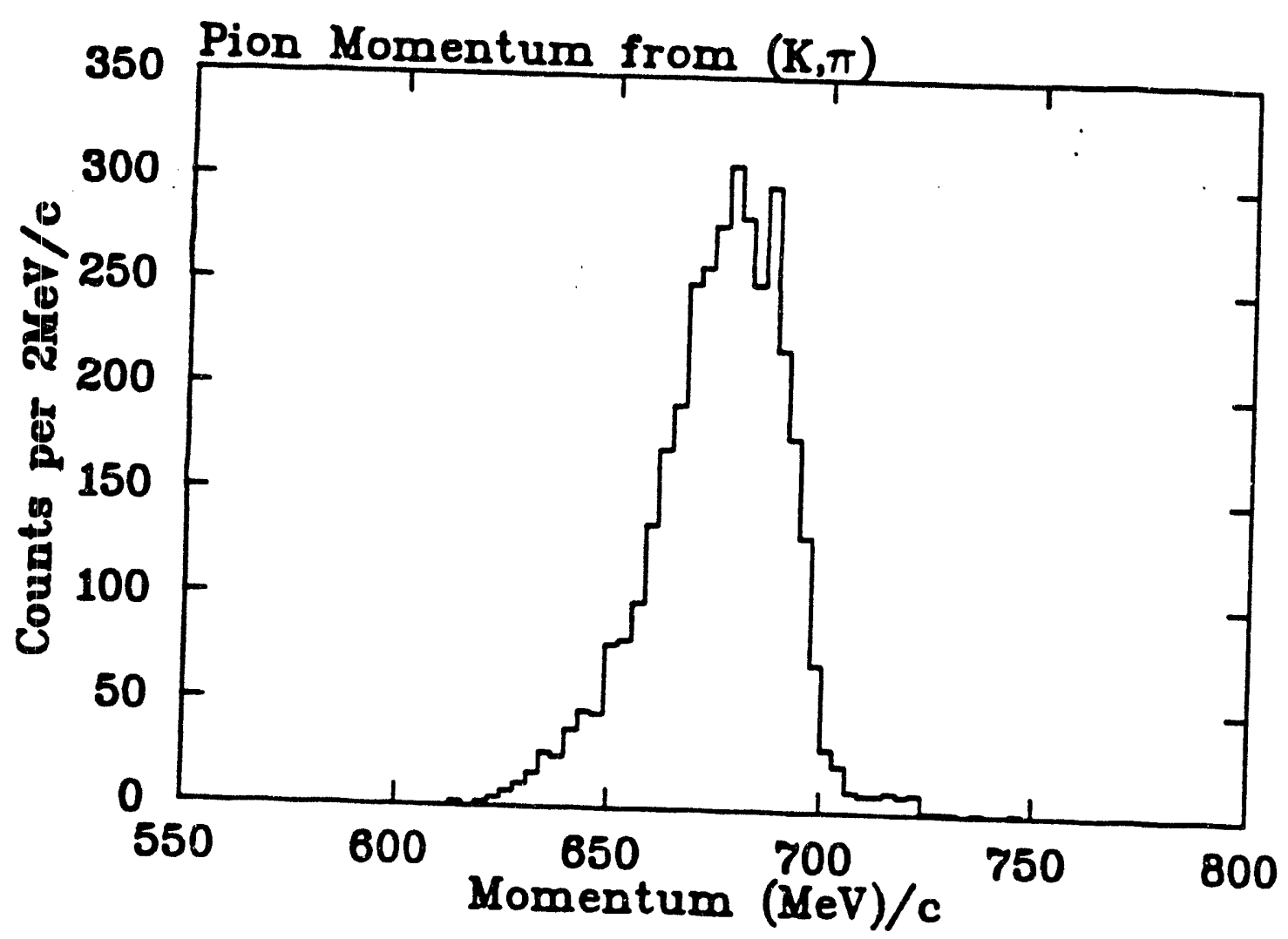

Figure 2.4 Pion momentum distribution as measured in downstream pion spectrometer. This particular event sample required a kaon incident upon the ${ }^{4} \mathrm{He}$ target and a charged track in the out-of-beam detector array. Also,
kaon decays, $K^{-} \rightarrow \pi^{-} \pi^{\circ}$ have been removed.

The readout system for the drift chambers was carefully designed to deal with the - low gain and high rates. Signal preamplifiers mounted directly on the chambers passed the signal to an amplifier and then to a discriminator. The output of this discriminator is continuously clocked into a First In First Out (FIFO) memory unit. The data is frozen in memory when a STOP signal is received from the trigger electronics. The resulting pattern passed to the acquisition computer represents the time the wires were hit and the duration for which the signal was above threshold. 

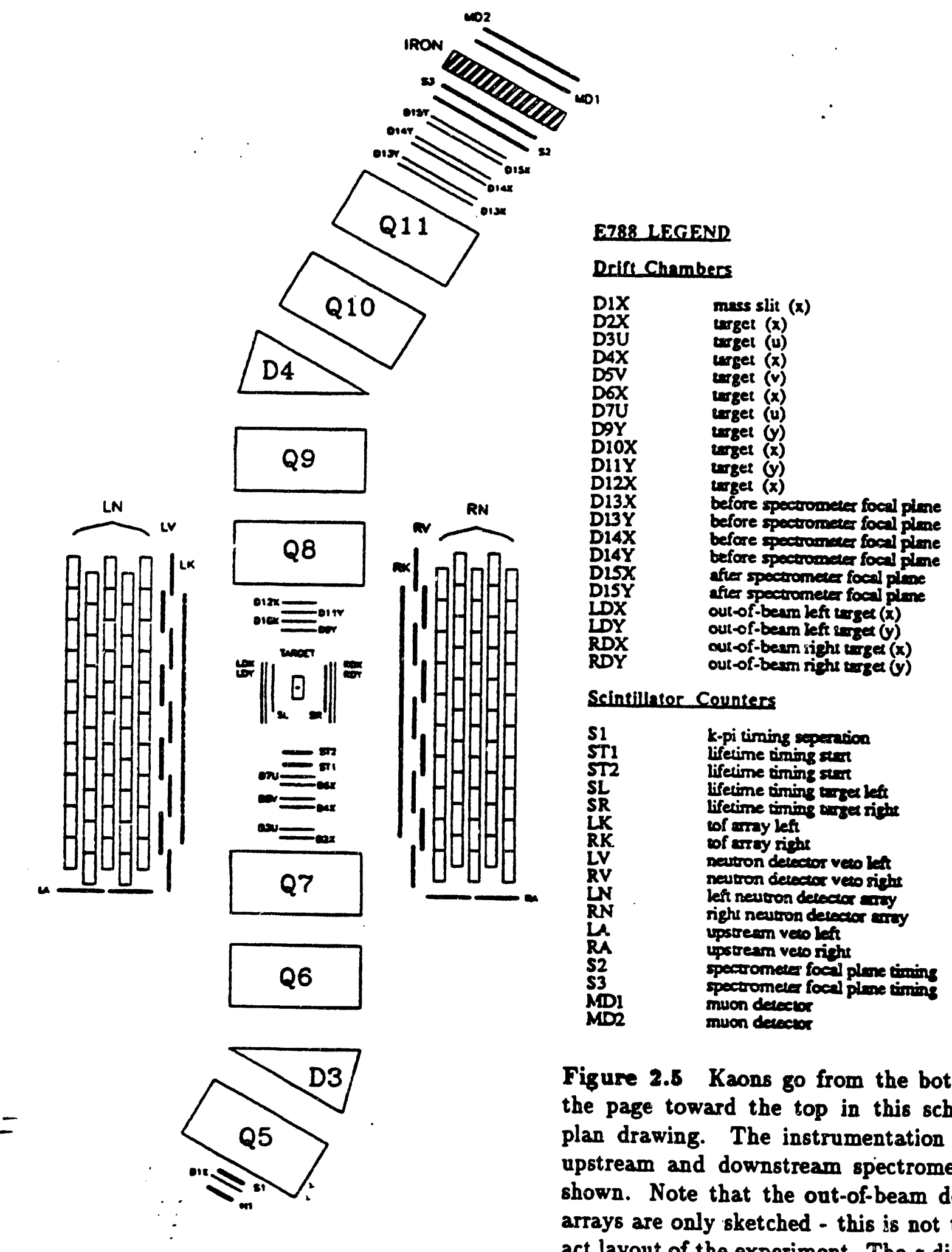

Figure 2.5 Kaons go from the bottom of the page toward the top in this schematic plan drawing. The instrumentation of the upstream and downstream spectrometers is shown. Note that the out-of-beam detector arrays are only sketched - this is not the exact layout of the experiment. The $z$ direction is defined along the beam axis, $y$ upward, and $x$ toward beam left 


\begin{tabular}{|c|c|c|c|}
\hline \multicolumn{4}{|c|}{ E788 Beam Instrumentation } \\
\hline Name & $z$ Position $(\mathrm{cm})$ & Orientation & Area \\
\hline Hodoscope & -455.6 & ' $x$ ' & mass slit \\
\hline $\mathrm{DCl}$ & -448.1 & $x$ & \multirow{7}{*}{ upstream target } \\
\hline $\mathrm{DC} 2$ & -75.8 & $x$ & \\
\hline DC3 & -71.7 & $\mathbf{u}$ & \\
\hline $\mathrm{DC} 4$ & -66.3 & $\mathbf{x}$ & \\
\hline DC5 & -62.3 & $\mathbf{v}$ & \\
\hline DC6 & -57.4 & $\mathbf{x}$ & \\
\hline DC7 & -53.4 & $\mathbf{u}$ & \\
\hline PIVOT & 0.0 & - & $\mathbf{z}=0$ position \\
\hline DC9 & 22.9 & $x$ & \multirow[t]{4}{*}{ down stream target } \\
\hline $\mathrm{DC} 10$ & 28.8 & $\mathbf{y}$ & \\
\hline $\mathrm{DCl1}$ & 32.5 & $x$ & \\
\hline $\mathrm{DC} 12$ & 37.0 & $\mathbf{y}$ & \\
\hline $\mathrm{DCl} 3$ & 41.4 & $\mathbf{x}$ & \multirow{6}{*}{$\begin{array}{c}\text { rear } \pi^{-} \\
\text {spectrometer }\end{array}$} \\
\hline $\mathrm{DC} 14$ & 621.6 & $x$ & \\
\hline DC14' & 624.2 & y & \\
\hline FOCUS & 659.4 & - & \\
\hline $\mathrm{DC} 15$ & 667.1 & $x$ & \\
\hline DC15' & 669.6 & $y$ & \\
\hline
\end{tabular}

Table 2.1 The location and orientation of drift chambers used in the ex-
periment E788. 


\subsubsection{Beamline Counter Elements}

\section{Mass Slit Hodoscope}

An eight element scintillator hodoscope was placed in the mass slit area just before DC1. There is an ambiguity which may be difficult to sort out for events with multiple hits in $\mathrm{DC1}$. Events of this type are eliminated from the primary trigger by requiring a single hodoscope element to fire.

\section{Precision Target Vertex Timing Scintillators ST1 and ST2}

The two ST counters were located $37.8 \mathrm{~cm}$ and $31.8 \mathrm{~cm}$ upstream from the pivot point. The scintillator material used for each ST was BC-418 of dimensions $15 \mathrm{~cm} \times 4 \mathrm{~cm} \times$ $0.5 \mathrm{in}$. The produced scintillation light has a fast characteristic rise time of $0.5 \mathrm{~ns}$ and fast decay time of $1.4 \mathrm{~ns}$. This makes it ideal for ultra fast and precise timing applications. All sides of the scintillator were polished and wrapped in light absorbing black paper. The XP2020 photomultiplier tube was chosen to be used at each end of each detector because of its high timing resolution characteristics. For proper operation in the relatively high rate environment near the hypernuclear production target $\left(\approx 3 \times 10^{6}\right.$ particles $/ \mathrm{sec}$ ), the last 3 dynodes of each base were voltage-stabilized with external high voltage supplies. Two identical counters close together were chosen to reduce the time resolution by a factor of $\sqrt{2}$.

The observed timing resolution of each counter is found to be $\sigma_{S T}=62.6 p s$ and described in more detail in section 3.3.1. The front-end instrumentation of the ST's is illustrated in figure 2.6.

\section{Upstream Timing Scintillator S1}

Beam pions and kaons are distinguished by velocity. The velocities of $750 \mathrm{MeV}$ kaons and pions are $25.05 \mathrm{~cm} / \mathrm{ns}$ and $29.5 \mathrm{~cm} / \mathrm{ns}$, respectively. The beam particle's velocity is determined from the time difference between the ST2 detector and another inbeam scintillator timing detector, S1. S1 is located $417 \mathrm{~cm}$ upstream of ST2. This corresponds to a flight time difference of $2.5 \mathrm{~ns}$ for pions and kaons. The front end electronics for the $\mathrm{S} 1$ detectors is shown in figure 2.6. 

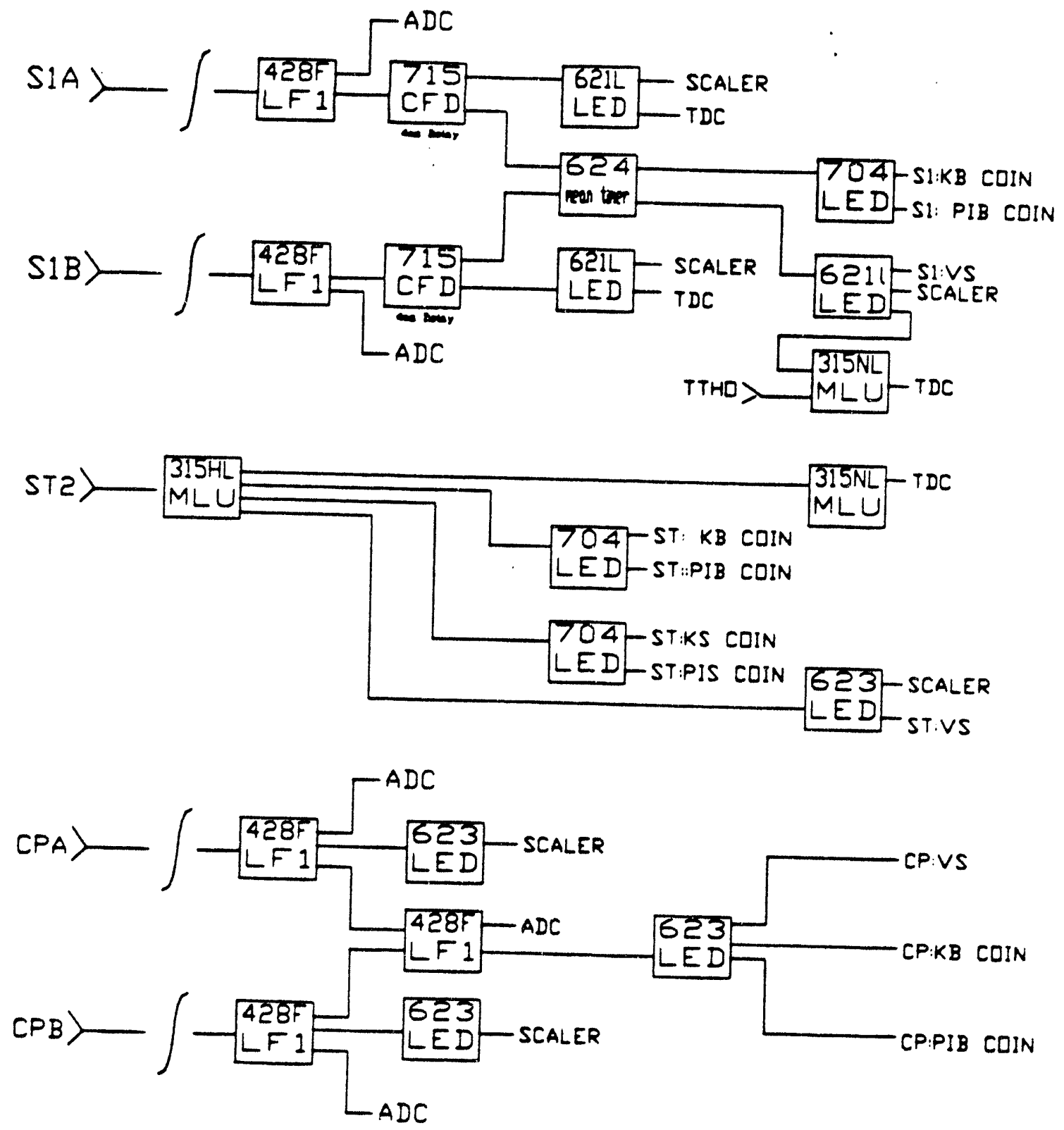

Figure 2.6 This schematic drawing illustrates the front end electronics used for the S1, ST, and Cerenkov detectors. 


\section{Critical Angle Pion Cerenkov}

The pions out number the kaons at the ST2 position by about 13:1. The success of the experiment relies upon the reduction of the pion background. A device called a critical angle pion Cerenkov counter was implemented to differentiate between pions and kaons.

As a charged particle passes through a medium at a velocity greater than the speed of light in that medium, it radiates light. This radiation is know as Cerenkov radiation. The angle which the light is emitted is related to the particle's velocity, $\theta_{C}=\cos ^{-1} \frac{1}{\beta n}$, where $\mathrm{n}$ is the index of refraction of the medium, and $\beta$ is the velocity fraction $\beta=\frac{v}{c}$. This means the faster the particle, the larger the angle of light emission.

The detector consists of a piece of lucite plastic with photomultiplier tubes optically coupled at either end. The plastic is wrapped in a light absorbing black paper. The index of refraction for lucite is $n=1.5$.

Light refraction at a boundary of two materials with indexes of refraction $n_{1}$ and $n_{2}$ is described by Snell's law,

$$
\frac{\sin \theta_{1}}{\sin \theta_{2}}=\frac{n_{2}}{n_{1}}
$$

where $\theta_{1}$ and $\theta_{2}$ are the angles with respect to the normal of the incident and refracted rays. When light is produced in the plastic radiator, it is either transmitted through the plastic/air interface or it is confined in the plastic by total internal reflection. Total internal reflection occurs for $\theta>\theta_{\text {crit }}$ where $\theta_{\text {crit }}$, the critical angle, is defined as the angle which gives $\theta_{2}=90^{\circ}$. This implies $\sin \theta_{c}=\frac{n_{2}}{n_{1}}$. This critical angle is related to a critical velocity fraction,

$$
\beta_{c}=\frac{1}{\sqrt{n^{2}-1}}=0.89
$$

A. $750 \mathrm{MeV}$ kaon and pion have velocity fractions of 0.84 and 0.98 , respectively. Therefore, light produced from a beam pion will produce total internally reflected light. In contrast, a beam kaon will not. Light detection by the photomultiplier tubes is then an indication that the beam particle is indeed a pion and should be discarded. The front end electronics for the pion Cerenkov is illustrated figure 2.6. 


\section{Downstream Timing Scintillators S2 and S3}

Timing scintillators S2 and S3 are placed in the rear of the pion spectrometer. Their combined role is analogous to the role of $\mathrm{S} 1$ in the upstream spectrometer; pions are identified by flight time between ST2 and S2 located $720 \mathrm{~cm}$ downstream from ST2.

The $x$ position of $\mathrm{S} 2$ also plays a crucial role in the momentum acceptance of the pion spectrometer. Since the momentum of the particle is roughly proportional to its $x$ position along the rear focal plane, the S2's placement acts as a window on the momentum acceptance.

\subsubsection{Trigger Level Electronics}

The identification of the particle incident upon the target is obtained from a time of flight measurement between S1 and ST2. Discriminated signals with narrow widths from the S1 and ST2 counters are aligned for pions and kaons by delay lines. If more than one element of the mass slit hodoscope is triggered, the event is discarded. This is done to reject multi-tracks incident upon the target.

A velocity measurement is made on the downstream trajectory, if it exists. Just as in the time of flight measurement between S1 and ST2, the downstream particle identification is established from a time alignment with the discriminated ST2 and S2 counter.

Trigger bits are set according to the possible permutations of the upstream and downstream identification: K-beam, PI-beam, KK, K-PI, and PI-PI. These trigger bits are used in conjunction with various requirements on the out of beam detector array. A logical OR is constructed to form the OBTRIG trigger bit.

\subsection{Cryogenic Liquid ${ }^{4} \mathrm{He}$ Target}

= The target area of the experiment is densely packed with counters, cryogenic equipment, and support structures. A wire frame design drawing of the region is shown in figure 2.7.

\subsubsection{Cryogenic Helium Target}

During the design phase of the cryogenic ${ }^{4} \mathrm{He}$ target, we had to keep in mind: 


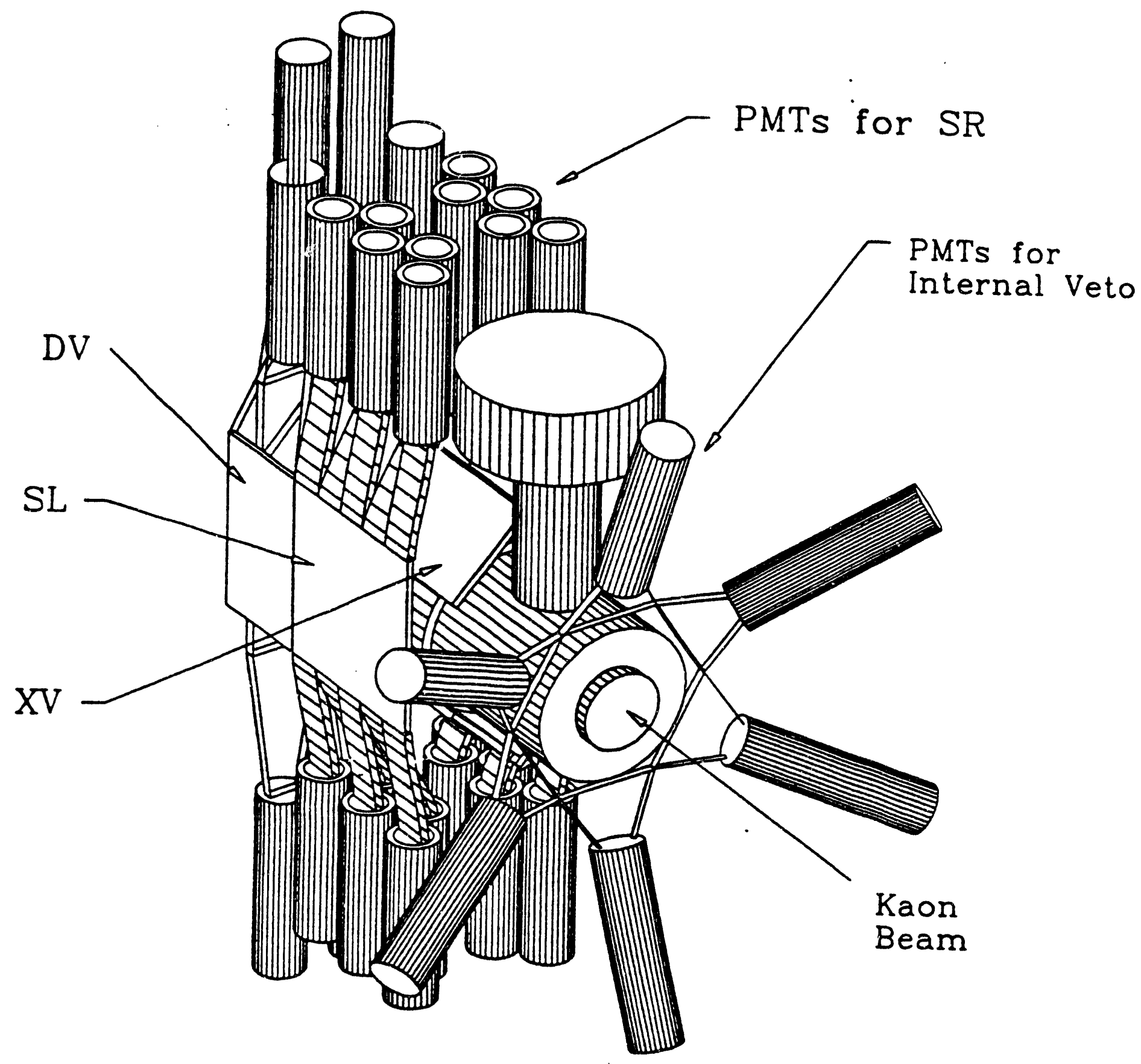

Figure 2.7 A wire frame design drawing of the E788 target region. The star configuration of cylinders on the upstream side of the target are the photomultipliers of the internal veto IV. 
- The low end of our energy acceptance is highly dependent upon the density and quantity of materials between the interaction point and the out-of-beam detector array. A balance was found between the high demands of a cryogenic system and optimizing physical measurements.

- Neutron production from pion absorbtion is a major background for the hypernuclear decay neutron measurement. This background is minimized when all $\pi^{-}$'s produced in coincident with the $\left(\mathrm{K}^{-}, \pi^{-}\right)$formation interaction are tagged. This becomes a formidable task when working in close proximity to the liquid Helium at $4^{\circ} \mathrm{K}$.

- The quality of kinematic measurements of the $\left(K^{-}, \pi^{-}\right)$interaction depend upon the amount and type of materials in the beam path. This requires a careful choice of vacuum windows, containment vessels, and thermal insulation.

- The quality of the hypernuclear lifetime measurement depends somewhat on how close the precision timing scintillators $S T$ and SL/R are placed to the $\left(K^{-}, \pi^{-}\right)$interaction region.

A schematic of our cryogenic target is illustrated in figure 2.8 .

One of the main features of the target design is the low density $\left(0.053 \mathrm{gm} / \mathrm{cm}^{3}\right)$ PVC foam support shell. The cylindrical shell has inner and outer radii of $3^{\prime \prime}$ and $4.5^{n}$, respectively. It has enough structural rigidity to contain the cryogenic vacuum within.

The Helium is contained by an 8 mil thick cylindrical shell of Dacron. The shell has dimensions of $2.5^{n}$ in diameter by $12^{n}$ in length. The vessel is filled by a copper $=$ pipe which attaches at the bottom and vented by a copper tube at the top. These fill and vent tubes are located very close to the upstream end of the vessel. The downstream end of the vessel is free from any plumbing.

The cryogenic containment vessel is iusulated from the PVC support shell by about 50 layers of super insulation. A layer of super insulation consists of a ultra thin aluminized mylar sheet together with a layer of acrylic gauze. 


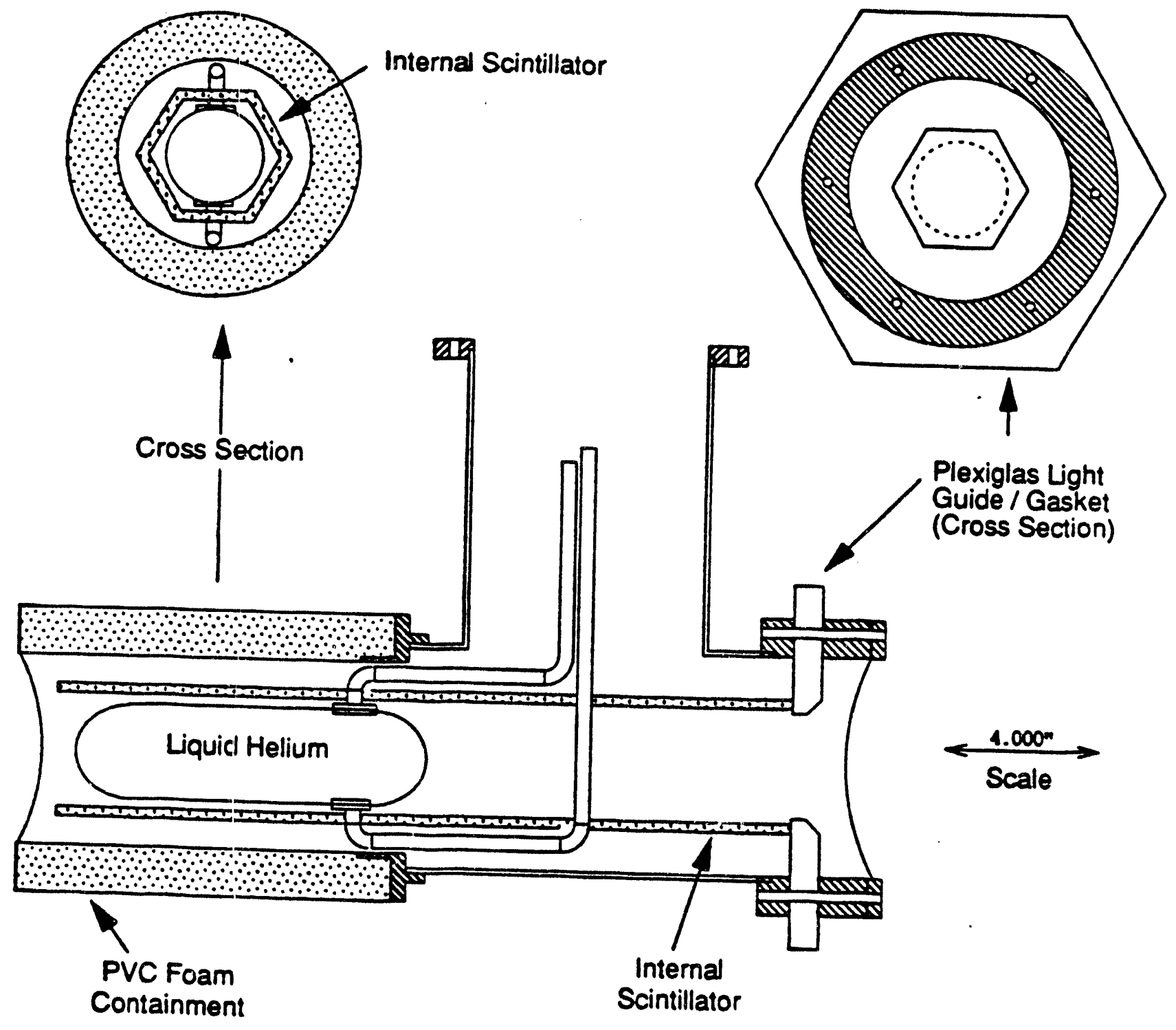

Figure 2.8 The kaon beam moves from right to left in this cross sectional view of the cryogenic target. The main features of this design include counters located inside of the cryogenic vessel to tag $\pi^{-}$emission. 


\subsubsection{Target Internal Veto System IV}

Pion production is tagged by a hexagonal shell of $\frac{1}{4}^{n}$ scintillator sheets inserted between the super insulation and PVC shell. The top and bottom scintillator sheets were slotted to accommodate the cryogenic plumbing. The four side sheets have a 'window' cut out of them for direct exposure of SL/R. A three dimensional design drawing is shown in figure 2.9. The light created in the scintillator is channeled out of the cryogenic vacuum by a lucite gasket. The inner radius of the gasket has hexagonal $45^{\circ}$ bevels to reflect the light out at $90^{\circ}$. Six external photomultiplier tubes (PMT's) are optically coupled to the gasket by adiabatic light guides. The resulting star pattern of PMT's is evident in figure 2.7. The signals from the PMT's are split by a 50:50 resistive divider. One signal is discriminated and fed to a CAMAC 50ns TDC and a FASTBUS pipeline TDC. The other signal is fed into a FASTBUS ADC.

\subsection{Target Out-of-Beam Counters}

\subsubsection{Target Veto Counters TV}

A system of scintillator type counters located outside of the PVC support shell act as a redundancy check for charged particle production inside the target. Two slabs of scintillator are placed at right angles to form a ' $\Lambda$ ' shaped roof on top of the PVC shell. Each slab is about $15 \mathrm{~cm}$ wide by $40 \mathrm{~cm}$ long and $\frac{3}{8}$ thick. Similar scintillator slabs are joined to make a ' $V$ ' shape and are located on the underside of the PVC shell. Light is channeled out of the scintillators by a right angle light guides. Photomultiplier tubes are optically coupled to the light guides.

There is a $12 \mathrm{~cm}$ gap between the downstream end of the target and the down-

- stream drift chambers. The gap is filled with two downstream scintillation type detectors DV. The detectors have dimensions $12 \mathrm{~cm} \times 12 \mathrm{~cm} \times \frac{3}{8}{ }^{n}$. They are coupled by adiabatic light guidés to a PMT at one end.

The signals from the PMT's are split by a 50:50 resistive divider. One signal is discriminated and fed to a CAMAC 50ns TDC and a FASTBUs pipeline TDC. The other signal is fed into a FASTBUS ADC. 


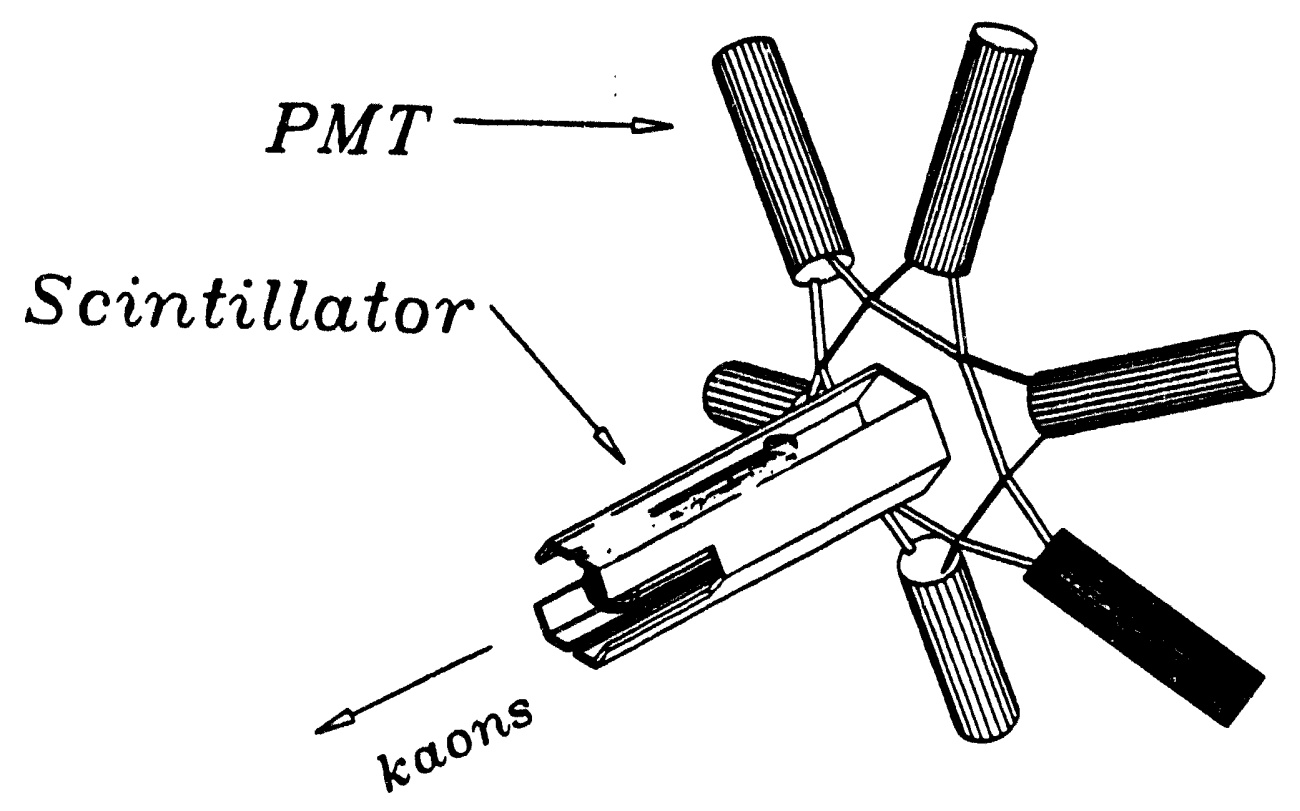

Figure 2.9 This three dimensional view shows the active elements used in the cryogenic ${ }^{1} \mathrm{He}$ target. The scintillator elements shown here were coupled to a lucite light guide gasket which channeled the light to external
photomultiplier tubes.

\subsubsection{Precision Target Scintillators SL/R}

The stop time for the hypernuclear lifetime measurement is measured directly by precision timing scintillator detectors located at either side to the target. They have the designated names SL and SR, referring to the beam left and right systems respectively (The potation SL/R refers to both detectors). Each side consists of five scintillator elements with dimensions $4.5 \mathrm{~cm} \times \frac{1}{4}$ in $\times 22 \mathrm{~cm}$. The first dimension is the width and is chosen to couple to a $2^{n}$ photomultiplier tube. Each end of the scintillator is coupled to RCA 8575 PMT's. The PMT's, like all others used in the experiment, were insulated from stray magnetic fields with $\mu$-metal shields. 


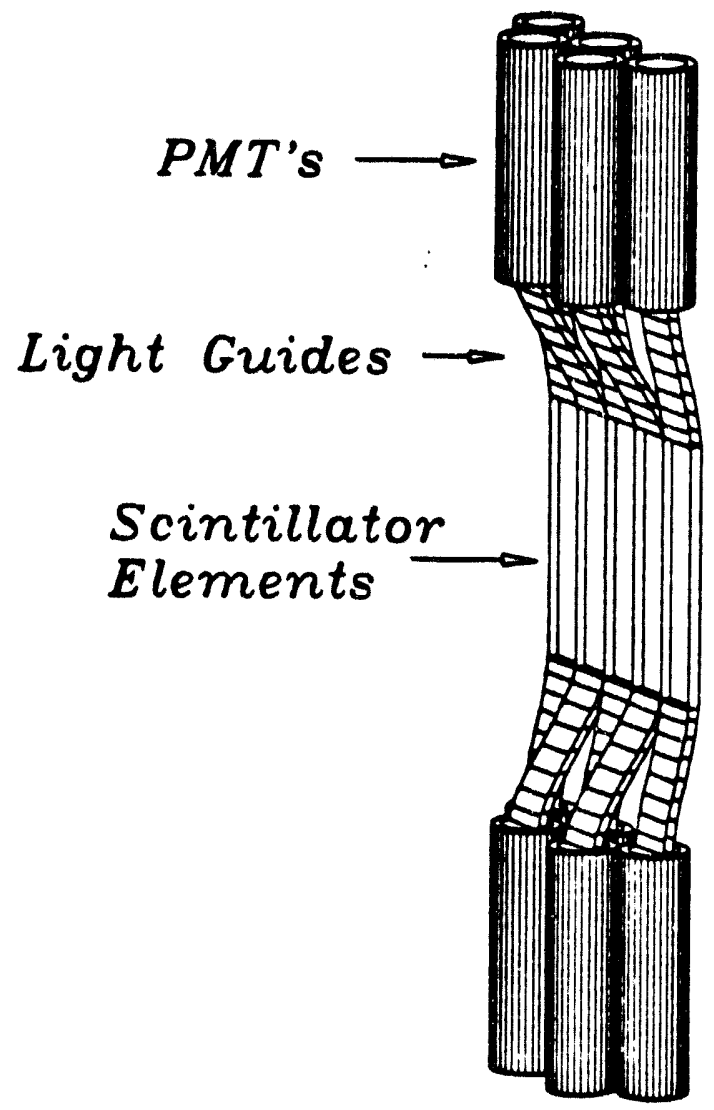

Figure 2.10 This design drawing of the SL counter illustrates how effcien ly space was used in the densely packed target area. The light guides of alternating detector elements are bent to allow close packing of the PMT's. This design also left the outside face of the assemblage flat to permit mounting to the out-of-beam drift chambers.

The light guides were made with Ultra-Violet Transmitting (UVT) plexiglas with cross section dimensions $4.5 \mathrm{~cm} \times \frac{3}{8} \mathrm{in}$. The thicker light guides compared to the scintillator optimizes light transmission from the scintillator. Tight packing of the PMT's was accomplished by making gentle bends in the light guides (see figure 2.10).

Each array was sandwiched between the cryogenic PVC shell and the out of beam drift chambers. In fact, the support structure of the SL/R was integrated into the drift chamber frame.

The signals from the PMT's were carried to the counting trailer by fast triax cable. Once there, the signals are split by a 20:80 resistive divider. The small split of 


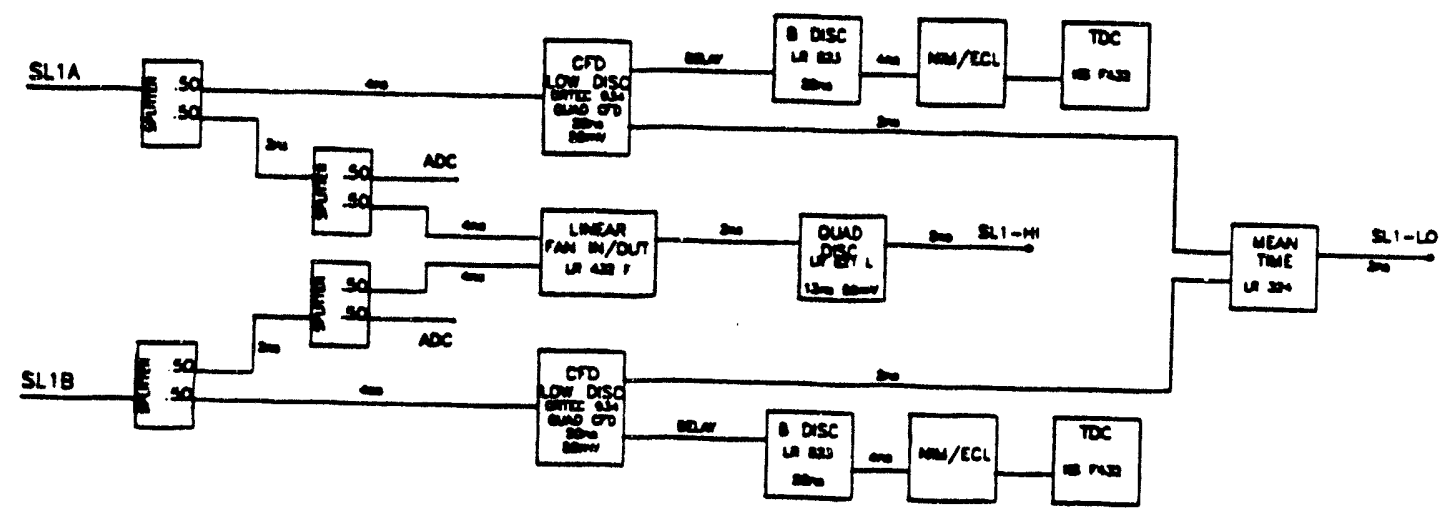

Figure 2.11 The two signals from the photomultiplier signals of one of the SL/R elements are shown in the left as SL1A and SL1B. The combined signals are discriminated at two different thresholds to produce the signals SL1-HI and SL1-LO. The higher threshold signals are associated with pro-
tons.

the signal is delayed and fed into a FASTBUS ADC. The larger split is discriminated with a constant fraction discriminator. The discriminated signals were then used in the trigger logic and also fed into FASTBUS TDC's. A schematic of the front end electronics used for the $\mathrm{SL} / \mathrm{R}$ counters is shown in figures 2.11 and 2.12

The SL/R scintillators are used not only for lifetime measurements but also for making charged particle velocity measurements. The velocity is determined from the time difference between the $\mathrm{L} / \mathrm{RK}$ and the SL/R (see figure 2.5). To complete the velocity measurement, good tracking is required to determine the flight path.

The $y$ hit position in the SL/ $R$ element can be derived from the time difference between the PMT signals at either end. A further discussion of time and energy calibration of the $S L / R$ is found in section 3.3. 

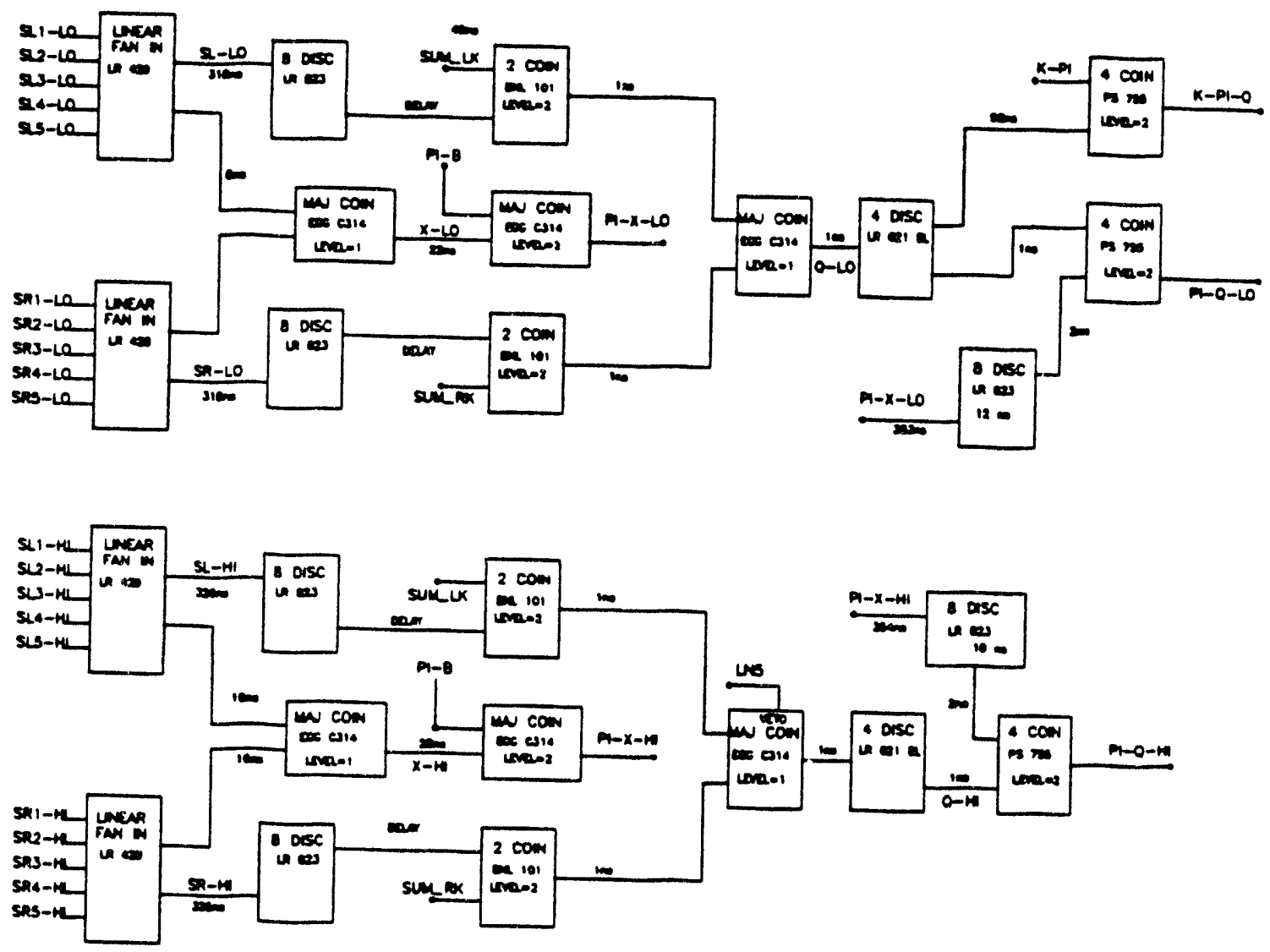

Figure 2.12 The hi and low discriminated signals from each SL/R element are brought together to form the triggers PI-Q-LO and PI-Q-HI. Also, the basic K-PI-Q trigger signal is constructed. 


\subsubsection{Out-of-Beam Tracking Chambers}

Dependable tracking of the out-of-beam trajectories is important for velocity measurements and determination of the hypernucleus vertex. For this experiment, a drift chamber was placed on each side of the cryogenic target adjacent to the SL/R arrays.

There are four sense wire planes in each drift chamber - two in $x$ and two in $y$ (the $y$ direction is defined upward, $z$ along the beam axis, and $x$ beam left using the right-hand convention). The wires in each plane are separated by $\frac{1}{2}$ in. The two $x$ planes are offset by $\frac{1}{4}$ in. Likewise, the two $y$ planes are offset similarly. This is done to increase the precision of the measurement in each plane.

Multiple hit position possibilities may exist because of an unresolvable ambiguity of which side of the sense wire the track passed. For such cases, the hit which is in best agreement with the hit in SL/R is chosen.

\subsection{Out-of-Beam Detector Arrays}

The primary task of the out-of-beam detector array is to identify and measure the hypernuclear decay products. There are two identical arrays located at beam left and beam right. The estimated solid angle coverage of the arrays is about $\frac{1}{3} \times 4 \pi$ steradians. Each array is composed of three types of counters. The arrangement of the arrays is illustrated in figure 2.13 .

Each element in the out-of-beam detector array is a scintillator type counter. The electric wake field of a charged particle causes ionization within the scintillator counter as it passes through. As the excited molecules de-excite into lower energy levels, light is emitted. It is this light which is detected by photomultiplier tubes coupled to the detector element.

\subsubsection{Layer One: L/RK}

The first layers of the out-of-beam array closest to the ${ }^{4} \mathrm{He}$ target are given the designated names LK and RK. LK is located on beam left, RK on beam right. Each layer is composed of ten scintillator elements of dimension $12 \mathrm{~cm} \times 1.5 \mathrm{~cm} \times 150 \mathrm{~cm}$. The elements are oriented with the long dimension vertical, as are all elements in the out-of-beam detector arrays. The top and bottom ends of the scintillators are coupled to photomultiplier tubes (PMTs) by trapezoidal plexiglass light guides. The 

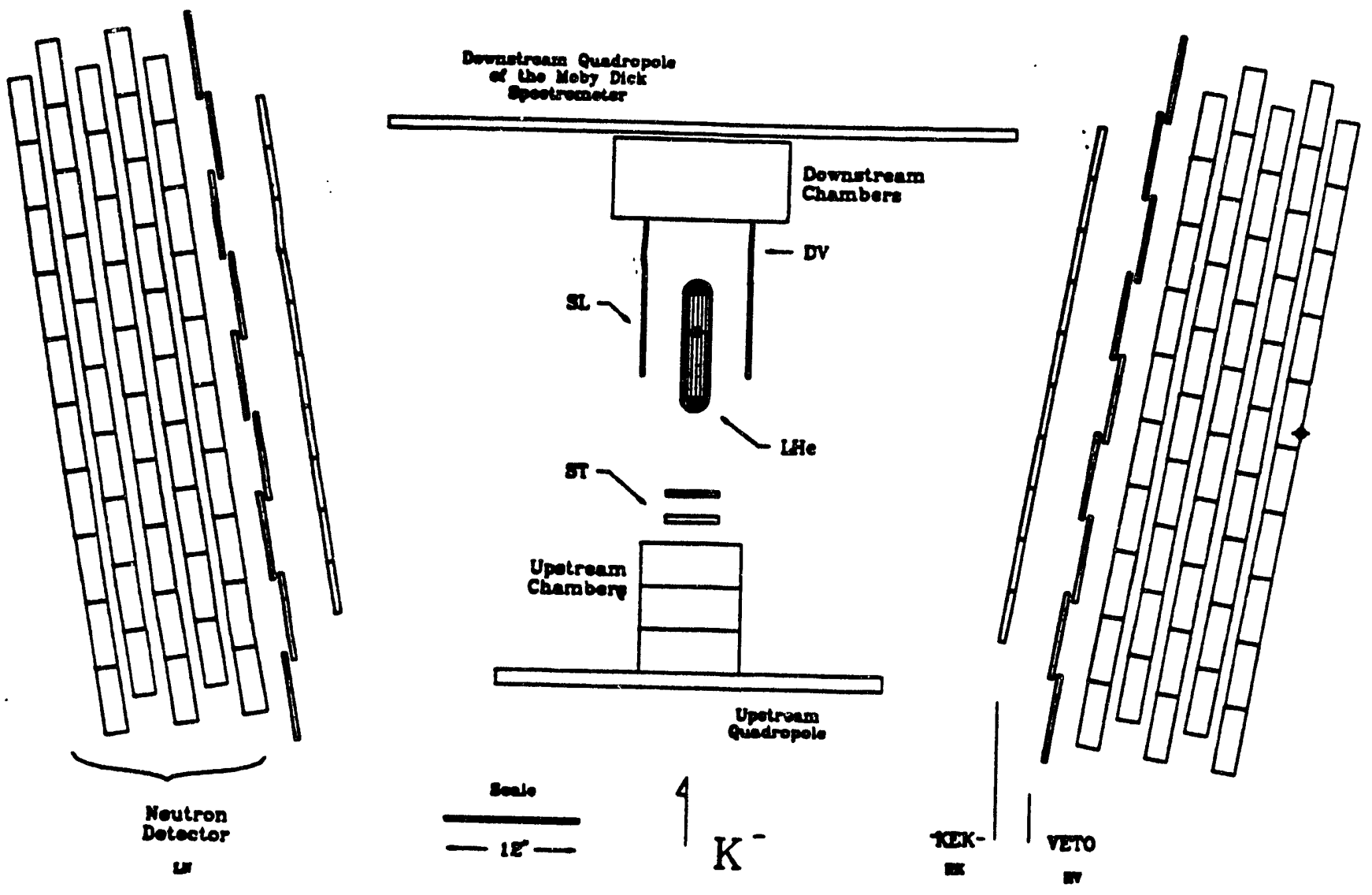

Figure 2.13 The placement of the out-of-beam detector arrays are illustrated in this plan view design drawing. A charged particle's velocicy is measured from the time of flight between SL/R and L/RK. The L/RV charge-particle veto maximized the neutron detection volume by tagging charged tracks destined to the L/RN. The L/RN layers are composed of 50 elements with an active volume of about 700 liters for each side.

PMT's were specially design by Hamamatsu for optimum time measurements. The scintillators and PMT's were used in a previous experiment at KEK in Japan. The detector elements were aligned side by side at a distance of about $80 \mathrm{~cm}$ from the ${ }^{4} \mathrm{He}$ target.

\subsubsection{Layer Tw̧o: L/RV}

For neutral particle detection in the neutron detector elements (next section) it is necessary to establish that hits in the neutron detector arrays did not originate from a charged particle. A large layer of counters was placed before the neutron detectors to 
intercept any charged tracks originating from the target area destined to the neutron detector array. These charged particle vetos are given the designated names LV and $\mathrm{RV}$ for the left and right side, respectively. There are nine elements in the veto layer - each with dimensions $9^{\prime \prime} \times \frac{3}{8}^{\prime \prime} \times 72^{\prime \prime}$. The elements are staggered and overlap about an inch. The elements are located $17.9 \mathrm{~cm}$ behind the L/RK layer. RCA 8578 photomultiplier tubes are coupled to each end via adiabatic light guides.

\subsubsection{Layers Three through Seven: $L / R N$}

Scintillating counters are not directly sensitive to neutral particles. The detection of neutrons relies upon nuclear reactions with the scintillator material. A nuclear reaction can release protons and alphas. Since these are charged, they will induce scintillation light which can be sensed by photomultiplier tubes.

There are fifty scintillator counters in each array. Each counter has dimensions $6^{\prime \prime} \times 2^{\prime \prime} \times 72^{\prime \prime}$. The scintillator type we used is BC-408. Each end of each element is coupled to Amperex 2262 PMT's via trapezoidal light guides. The elements are arranged in five layers which are staggered by $3^{\prime \prime}$. The first layer is located $23.9 \mathrm{~cm}$ behind the L/RK layer. The neutron detector layers are separated by about $2.5 \mathrm{~cm}$. The total active volume for neutron detection for each side is $4320 \mathrm{in}^{3}$. The probability for an $80 \mathrm{MeV}$ neutron to interact within a $2^{\prime \prime}$ distance is about $6 \%$. Details of the counters neutron detection efficiency is described in section 3.5. A schematic of the front end electronics used for the neutron detectors is shown in figures 2.14 and 2.15.

The detectors were thoroughly tested before implementation. The timing resolution obtained in tests performed at the CMU lab were typically about $300 \mathrm{ps,}$ FWHM, for $10 \mathrm{MeV}$ energy deposited.

\subsubsection{Additional Elements}

Scintillator type counters identical to the L/RV elements were placed on the upstream faces of the out-pf-beam detector arrays. Charged tracks emanating from the beam source direction towards the neutron detectors are tagged. These auxiliary veto counters have the designated names L/RA.

Two elements of dimension $11 \mathrm{~cm} \times 1.5 \mathrm{~cm} \times 150 \mathrm{~cm}$ were placed horizontally across the light guides and PMT's of L/RK. These were intended to define the solid angle 

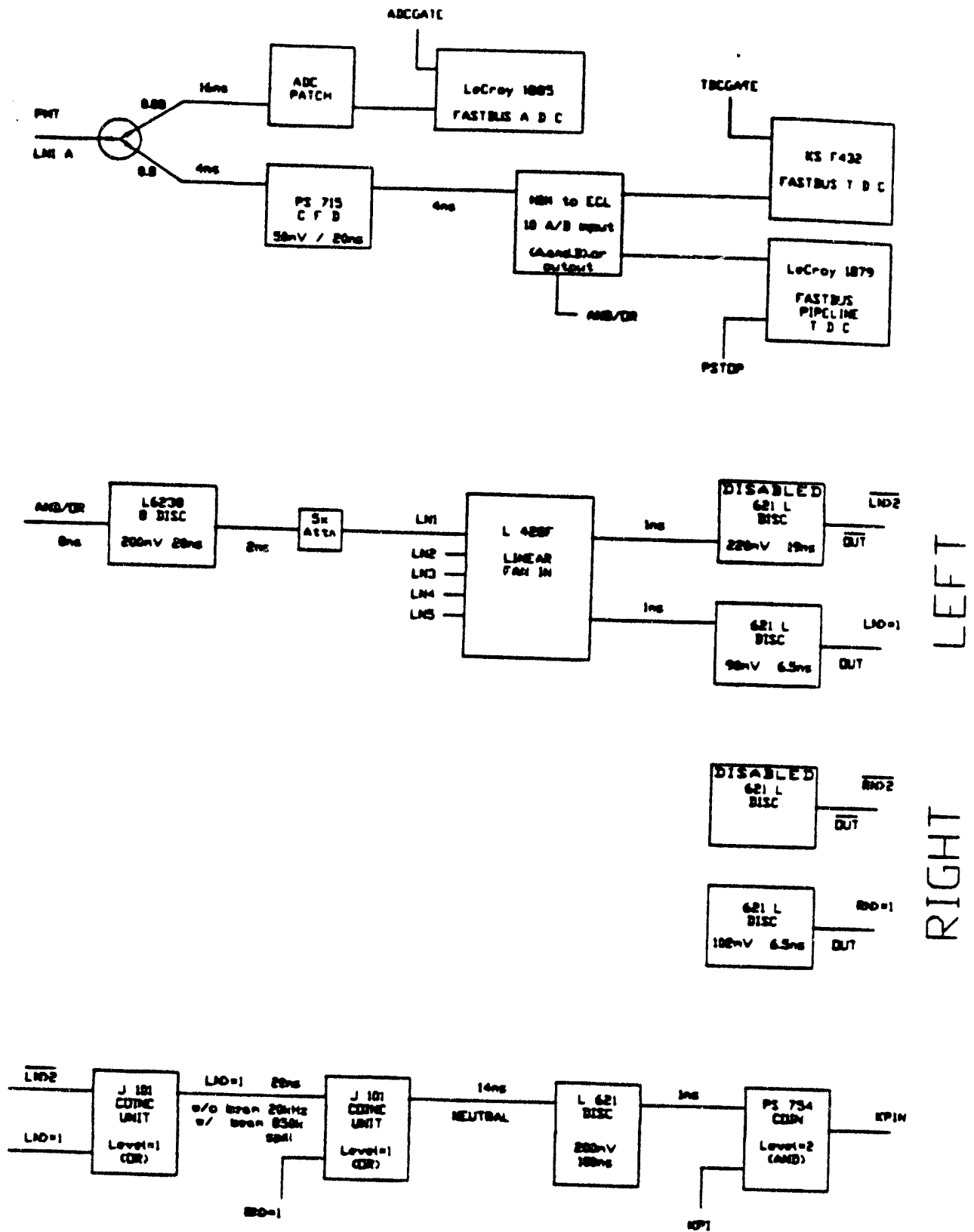

Figure 2.14 A signal from one of the photomultiplier tubes of the $L / R N$ arrays is input to a signal splitter as LN1A (upper left). A trigger bit is formed from the OR of all the ANDs of the two PMT signals to form a trigger bit which indicates whether a neutron detector was hit. 

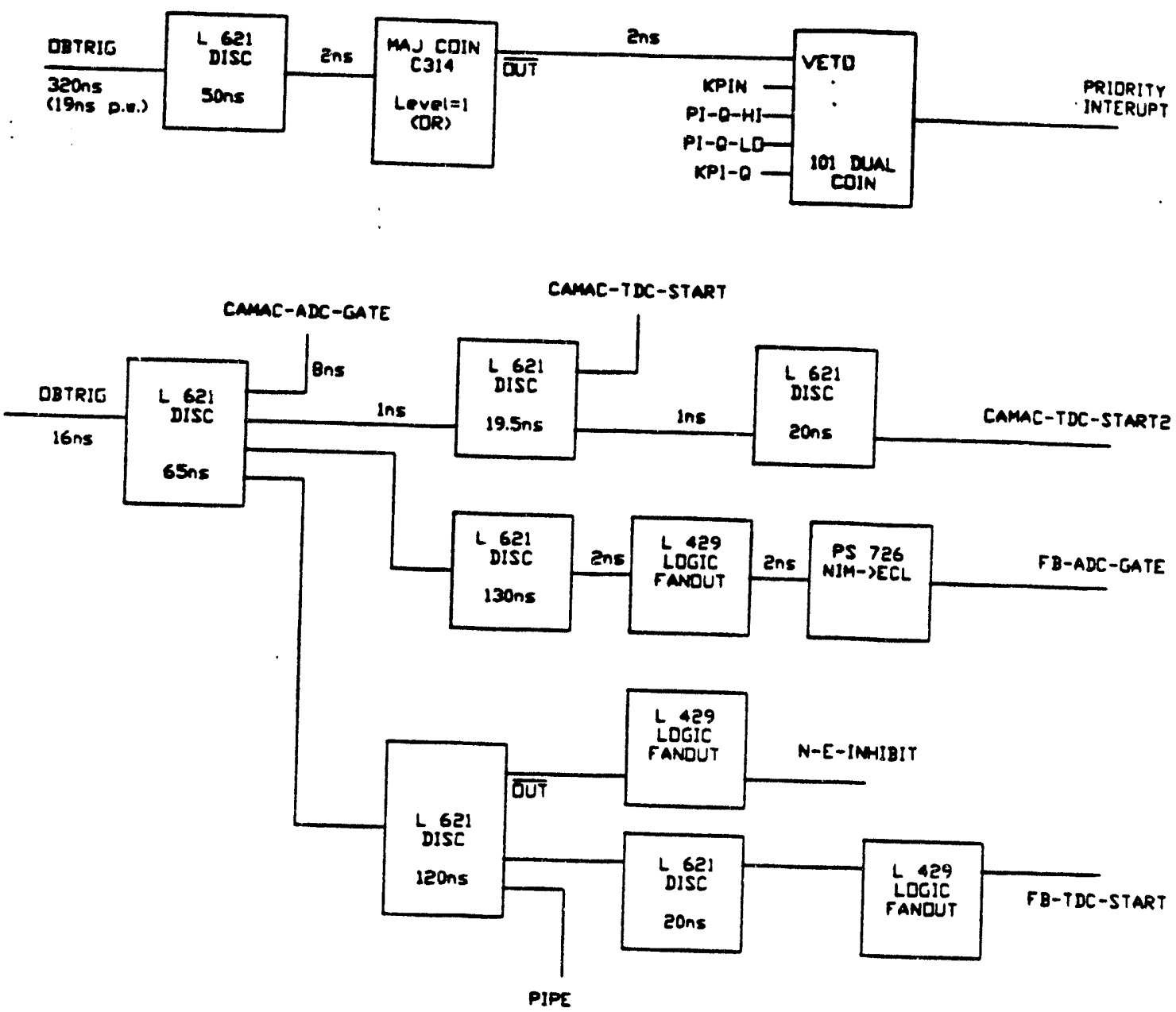

J

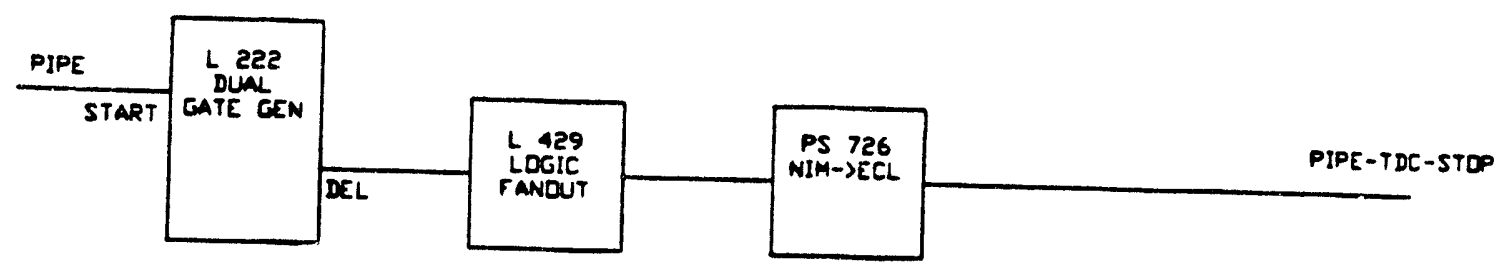

3

Figure 2.15 The gates for the acquisition electronics are schematically drawn. The signal OBTRIG is the logical OR of the various triggers involving the out-of-beam detector array. 
of charged particle trajectories into the out-of-beam detector arrays. These detectors were not utilized in the analysis.

\subsection{Measurement Electronics}

FASTBUS measurement electronics was used on almost every PMT signal in the experiment. FASTBUS is an engineering instrumentation standard which features very high data transfer rates, a cost effective high channel density, and permits fast bus control through processor or sequencer. FASTBUS is the successor to the 1960's CAMAC standard.

\section{Energy Measurements}

Individual photomultiplier tube signals are split by $50 \Omega$ voltage divider networks which result in a $0.08: 0.8$ division. The larger proportion is sent to make time measurements. The smaller proportion is delayed and fed into LRS $1885 \mathrm{~N}$ FASTBUS Analog to Digital Converters, (ADC). These 96 channel devices have a 12 bit resolution. The calibration of the devices is describe in detail in section 3.3.2.

\section{Time Measurements}

The accuracy of many of the time measurements in this experiment was critical to it's success. Details such as the method of discriminating signals were dealt with carefully.

The output of a leading-edge discriminator depends whether the input signal surpassed a set threshold voltage. The amplitude of the signal input is compared to a reference threshold voltage. If the signal input amplitude becomes greater than the reference threshold reference, the logical state of the output is switched for an adjustable amount of time.

The integrated charge coming from the photomultiplier tubes varies greatly depending upon the amount of light sensed. The relative time at which the discriminator triggers will differ depending upon the size of the signal. This results in energy deposition dependent timing. In principal, this energy dependence is removable in software. 
In this experiment we used Constant Fraction Discriminators (CFD's) on every PMT signal (except the IV and TV detectors). The input analog signal is split. One of these signals is then delayed and inverted while the other is attenuated by a factor $f$. These two pulses are added producing a bipolar signal, which goes through zero at a point were the initial signal reached a fraction $f$ of its total height. The final stage of this device is a zero crossing discriminator which produces a logic pulse at the output when zero is detected in the bipolar pulse. The benefit of CFD discrimination is the trigger time is independent of the pulse height (energy deposition independence).

The output levels of the CFD's are NIM. A conversion to ECL levels must be made to couple to the FASTBUS TDC's. The specialized devices used to make this conversion also perform a primary check of whether a detector layer was triggered. A logical AND is performed upon the two PMT signals on each detector element. For each layer, a logical OR is performed upon these AND's to form a trigger bit which indicates whether the detector layer was hit.

The NIM to ECL converters also had a master inhibit input. We used this input to control the time window for which the TDC's were permitted to receive signals. This time window was adjusted to $80 \mathrm{~ns}$. This is then the live-time per event of the detector system.

The ECL signals were digitized by Kinetic Systems F432 Time to Digital Converters (TDC's). Each 64 channel module is capable of making time measurements with an accuracy of $\sigma=25$ picoseconds. Its 12 bit range translates into a full scale time range of $100 \mathrm{~ns}$. They feature an on board range calibration/stabilization corrections via a ROM lookup table. A verification of the calibration is described in section 3.3.1.

\section{Pipeline TDCs}

$\mathbf{3}$

In the course of the experiment it was necessary to monitor well over 300 photomultiplier signals. A quick way to monitor each detector is to look for changes in it's hit rate. Typically, a drop in rate indicates a malfunctioning PMT while an increase in rate points to a light leak. The large number of channels prohibited the use of CAMAC scaler modules.

For this experiment, we exploited a device called a pipeline TDC (LRS 1879). Each of the 96 input channels is a 512 bit shift register. As an input receives a hit, a 
bit is set in the channels register. The time interval for which the register is shifted becomes the bin to bin time resolution. The shift rate is programmable and was set to give $4 \mathrm{~ns}$ bins. This gives a full scale of $2 \mu \mathrm{s}$. The device is designed primarily for drift chambers, microstrip detectors, or such high rate devices.

The pipeline TDC continuously accepts data and behaves in a first-in-first-out cycle fashion. Once the devices are stopped and read out, the shift registers are dumped and give a hit history for the previous $2 \mu \mathrm{s}$. The hit rate is then determined from the number times the detector is hit for each read out.

The devices were further exploited. An attempt was made to distinguish $\pi^{-}$from $\pi^{+}$in the out-of-beam detector array by taking advantage of

- $\pi^{+}$'s are not attracted to nuclear cores as they come to rest in a material unlike $\pi^{-}$'s

- The $\pi^{+}$decays into $\mu^{+}+\bar{\nu}_{\mu}$ with a mean life time of $26 \mathrm{~ns}$. The $\mu^{+}$subsequently decays via $e^{+}+\bar{\nu}_{e}$ with a mean lifetime of $2.2 \mu \mathrm{s}$. The $e^{+}$has an energy of about $50 \mathrm{MeV}$

Thus, $\pi^{+}$'s are identified by looking for multiple pulses in the identified pion's trajectory. The pipeline TDC's are well suited for making multi-hit timing measurements. 


\section{ANALYSIS}

A data analysis includes all the processes necessary to transform information recorded by the experimental apparatus into physics results. This task includes 1) transformation of the information encoded data tapes to ADC, TDC, and scaler digitized information, and 2) calibration of all digitized information into actual physical quantities such as position, time, energy, momentum, etc... The focus of this chapter is the second item - calibration of the detector apparatus.

\subsection{In-Beam Analysis}

\section{Background Reduction}

The composition of the beam at the point of ST2 $(31.8 \mathrm{~cm}$ upstream from the center of the liquid ${ }^{4} \mathrm{He}$ target) is dominated by pions. The $\pi^{-}$to $K^{-}$ratio was typically 13:1 through out the experiment. The selection of the particle incident upon the ${ }^{4} \mathrm{He}$ targét is made at the trigger level by, (1) a critical angle Cerenkov counter triggered on pions, and (2) a time-of-fight cut between S1 and ST2. This method is very effective for reducing background before ST2; however, this method is ineffective for kaon decays past ST2. 
A $K^{-}$will decay primarily through the channels

$$
\begin{array}{rr}
K^{-} \rightarrow \mu^{-}+\bar{\nu}_{\mu} & 63.5 \% \\
\pi^{-}+\pi^{\circ} & 21.2 \%
\end{array}
$$

with a mean lifetime[2] of $12.371 \pm 0.029$ ns. For a kaon momentum of $750 \mathrm{MeV}$, this corresponds to a mean distance of $\lambda=563 \mathrm{~cm}$. The decay fraction, $e^{\frac{-\pi}{\lambda}}$, at the target is $9.4 \%$. That is, about 9 out of every 100 kaons identified at ST2 will have decayed by the time they reach the center of the target area, $37 \mathrm{~cm}$ downstream of ST2. What really makes this a problem is that the decay $\pi^{-}$or $\mu^{-}$can remain within the acceptance of the downstream spectrometer and can then be mistaken for a $\pi^{-}$ originating from a $\left(\mathrm{K}^{-}, \pi^{-}\right)$reaction.

Fortunately, the $K^{-}$decay kinematics are distinguishable from the $\left(K^{-}, \pi^{-}\right)$ interaction. Figure 3.1 shows the predicted momentum difference dependence of the scattering angle between the front and rear spectrometer tracks. The curve is based only upon kinematics. The same plot for $\left(\mathrm{K}^{-}, \pi^{-}\right)$triggers in coincidence with a charged track in the out-of-beam detector array is shown in figure 3.2. Also displayed in figure 3.2 is a two dimensional cut made in this plane to eliminate the kaon decay background.

\section{In-Beam Kinematics}

In order to get a kinematic description of the $\left(\mathrm{K}^{-}, \pi^{-}\right)$reaction, we rely upon the upstream $\left(K^{-}\right)$and downstream $\left(\pi^{-}\right)$spectrometers to measure the kaon momentum $p_{K}$, the pion momentum $p_{\pi}$, and the scattering angle between the two trajectories $\alpha$. The hypernuclear excitation energy is then calculated from

$$
=E E=\left[\left(\sqrt{p_{K}^{2}+m_{K}^{2}}-\sqrt{p_{\pi}^{2}+m_{\pi}^{2}}+M_{4} \mathrm{He}\right)^{2}-\left(p_{K}^{2}+p_{\pi}^{2}-p_{K} p_{\pi} \cos \alpha\right)\right]^{\frac{1}{2}}-M_{\Lambda} \mathrm{He}
$$

The magnetic spectrometer facility used in this experiment has been used in many similar experiments. Its use and performance is well documented, for example, in the Ph.D. dissertations of Grace[7] and Szymanski[8]. I will avoid a detailed discussion of the spectrometer and only briefly describe pertinent information for this analysis. 


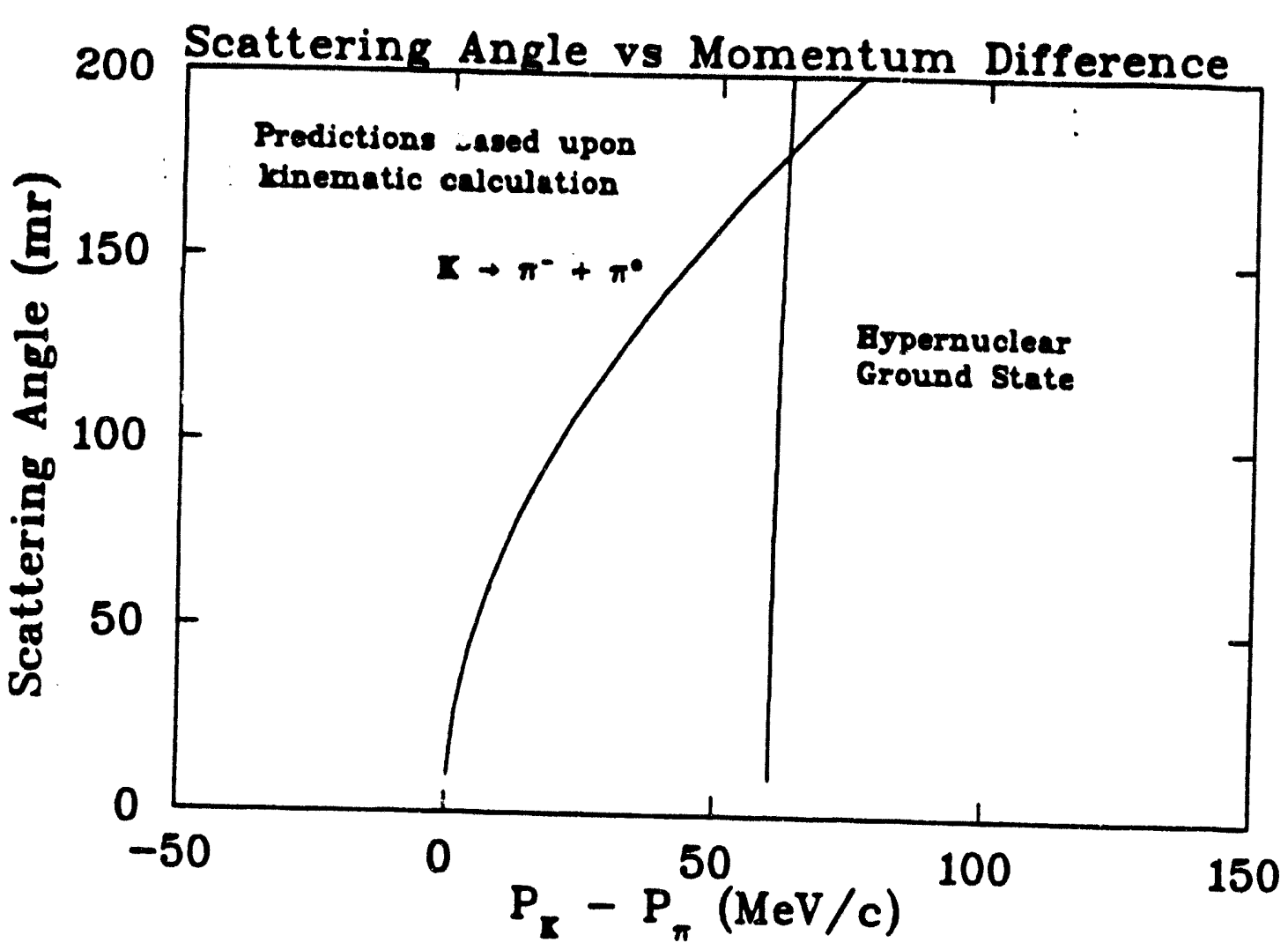

Figure 3.1 The predicted angle between the front and back tracks is plotted as a function of the momentum difference between the two tracks. This is done for two cases; (1) the $K^{-}$decay $K^{-} \longrightarrow \pi^{\circ}+\pi^{-}$, and the $\left(K^{-}, \pi^{-}\right)$interaction for ${ }_{\Lambda}^{1}$ He production.

The trajectory of a charged particle passing through a magnetic field is determined by the Lorentz force equation

$$
\mathbf{F}=\frac{d \mathbf{p}}{d t}=q \mathbf{v} \times \mathbf{B}
$$

This expression can be solved numerically but is CPU intensive and thus, not the method of choice. Alternatively, we use the TRANSPORT formalism which is analogolous to the matrix method employed in simple optical systems. The method is based upon a secund order matrix expansion of the coordinates $V=\{x, y, \theta, \phi, \delta\}$ where $x$ and $y$ are the orthogonal coordinates to the beam axis $(z), \theta=\tan ^{-1}\left(\frac{d x}{d z}\right)$, $\phi=\tan ^{-1}\left(\frac{d y}{d z}\right)$, and $\delta$ is the percentage momentum deviation from the central momentum. A transformation of vector $V$ to another coordinate in the spectrometer $V^{\prime}$ 


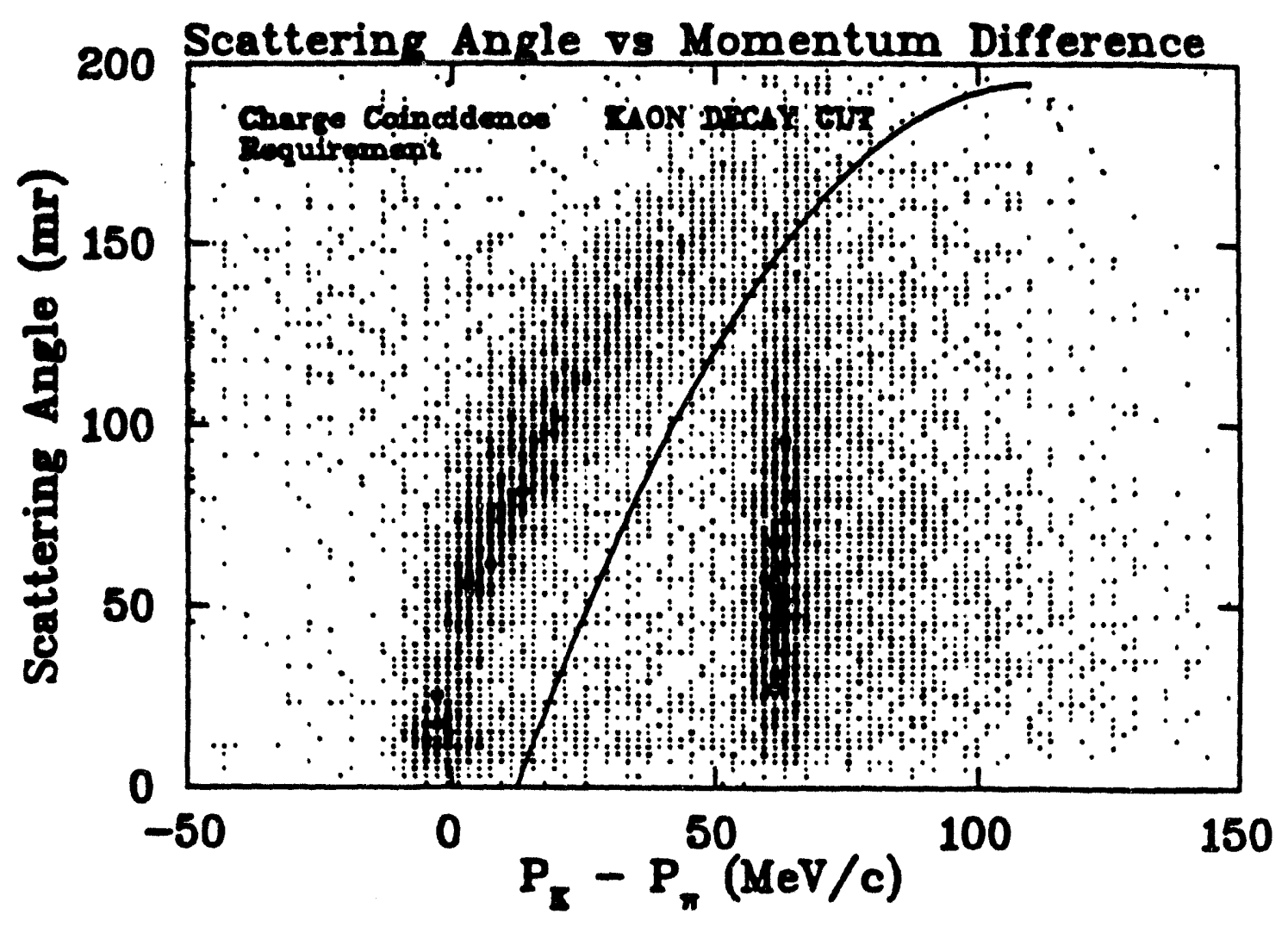

Figure 3.2 The scattering angle between the front and back tracks is plotted as a function of the momentum difference between the two tracks for raw $\left(K^{-}, \pi^{-}\right)$triggers. A comparison with figure 3.1 shows that the events from the decay $K^{-} \rightarrow \pi^{0}+\pi^{-}$are well separated from the hypernuclear formation $\left(K^{-}, \pi^{-}\right)$events. Also shown is the cut made in this plane to eliminate kaon decays.

is calculated by

$$
V_{i}^{\prime}=\sum_{j} M_{i j} V_{j}+\sum_{j, k} T_{i j k} V_{j} V_{k}
$$

$=$ where $M_{i j}$ and $T_{i j k}$ are first and second order transformation matrices determined by the spectrometer configuration. This relation is inverted so that the momentum fraction $\delta$ can be determined, provided enough information exists.

The energy resolution is determined from a Gaussian fit to the excitation energy (equation (3.1)) with a proton coincidence in the out-of-beam detector array (see section 3.1). The measured resolution is $\sigma_{E}=1.86 \mathrm{MeV}$. 


\subsection{Data Reduction}

Each of the 140 valid 9-track 6250 bpi data tapes holds about a hundred thousand events (see section 2.2.3 for details of the composition of the bulk data). A very large proportion of this data has poor and irresolvable in-beam tracking. These events are characterized by a series of hits in the drift chambers inconsistent with a continuous single track. Possible sources of these unreconstructable tracks include (1) $\mathrm{K}^{-}$decays, (2) $\pi^{-}$decays, (3) background accidental hits in the chambers, (4) multiple tracks, and (5) insufficient hit information to make a momentum measurement. Since we are not attempting an absolute cross section measurement, we are only interested in producing an unbiased sample of hypernuclear events. Details of in-beam tracking inefficiencies are not pertinent as long as they do not bias the hypernuclei sample.

In the course of the data analysis we repeatedly process the entire data set many times. To increase our productivity we created a reduced data set which is a subset of the raw data with the elimination of the obviously bad events. A redundancy is built into the in-beam tracking system. Measuring both position and direction of the track at the entrance and exit of the spectrometer over-determines the fit of the momentum. Large residuals in the difference of the predicted and measured is an indication of a troubled track. Figure 3.3 illustrates the distribution for the residual (measured minus predicted) for the $y$ measurement made at the rear of the downstream pion spectrometer.

Potential $\left(\mathrm{K}^{-}, \pi^{-}\right)$events which lack a vertex in the target area are discarded and are of no use to the analysis. The ${ }^{4} \mathrm{He}$ target is $30 \mathrm{~cm}$ long. Since the in-beam vertex $z$-resolution is poor (because of the small scattering angle), we conservatively accept events with $z$-coordinate between $-50 \mathrm{~cm}$ to $40 \mathrm{~cm}$. The raw $z$-coordinate for the raw $\left(\mathrm{K}^{-}, \pi^{-}\right)$event sample is shown in figure 3.4.

A substantial number events manage to punch through the ${ }^{4} \mathrm{He}$ target without interaction and mimic a $\left(\mathrm{K}^{-}, \pi^{-}\right)$tag. These events are characterized by a very small scattering angle and a large and negative momentum difference. A two dimensional cut on the scattering angle verses momentum difference plane is made for $0 \mathrm{mr} \geq \alpha \geq$ $25 \mathrm{mr}$ and $-15 \geq p_{K}-p_{\pi} \geq 15 \mathrm{MeV}$. 


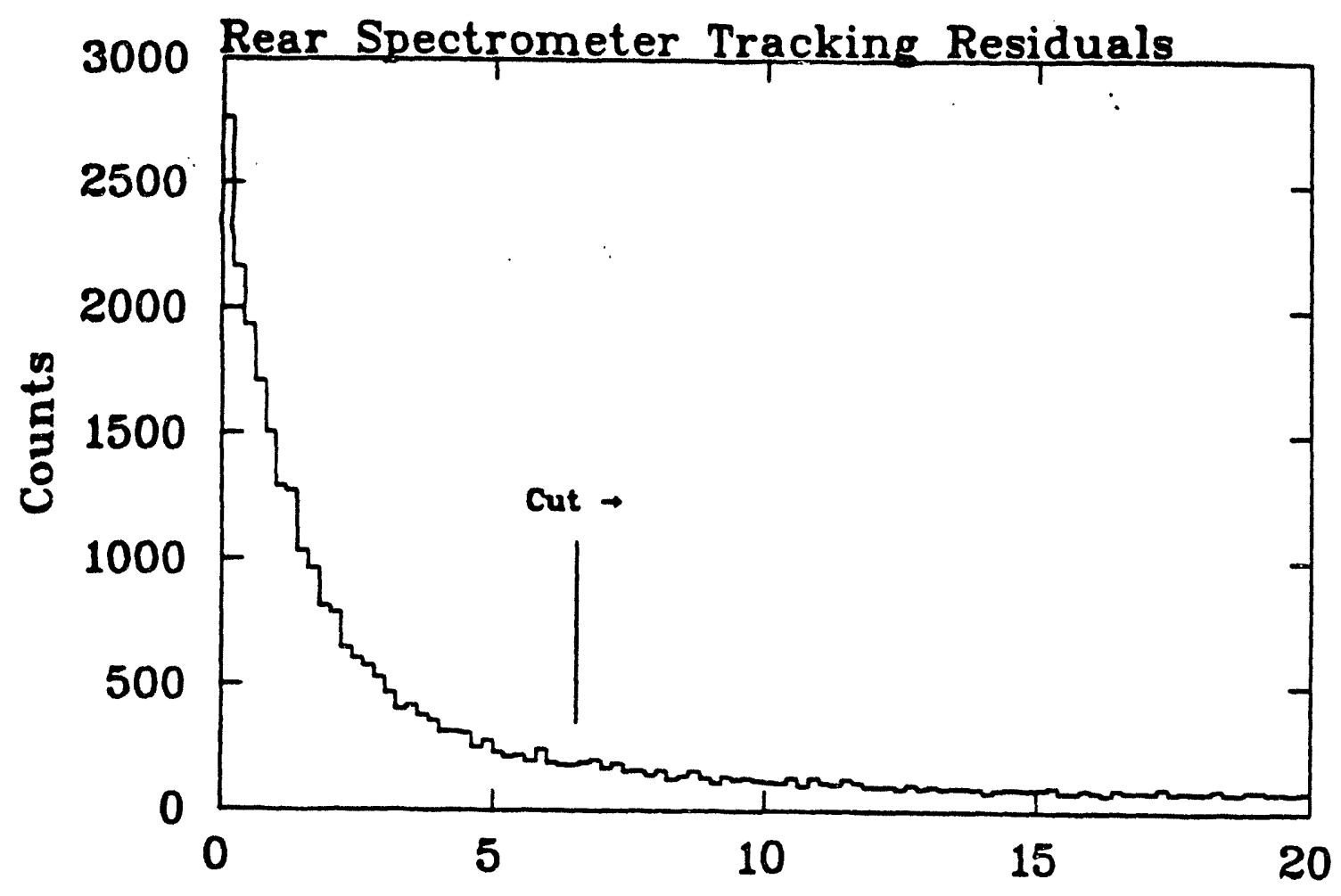

Figure 3.3 Shown is a histogram is of the difference between the measured $y$-coordinate at the rear of the downstream pion spectrometer minus the predicted value determined from tracking at entrance of the spectrometer. Also illustrated is the cut made for the initial data reduction.

\subsection{Out-of-Beam Detector Calibration}

The out-of-beam detector array consists of 138 separate scintillator elements with two photomultipliers each. Each photomultiplier is connected to an Analog to Digital Converter (ADC) and a Time to Digital Converter (TDC) (details are discussed in section 2.6 ). The time and energy calibration is described in the following sections.

\subsubsection{Time Cạlibration}

Every detector in the out-of-beam detector array has two photomultiplier tubes one at either end. Therefore, two calibration constants must be obtained for a total calibration. In the analysis of this experiment we are only interested in ; (1) the time 


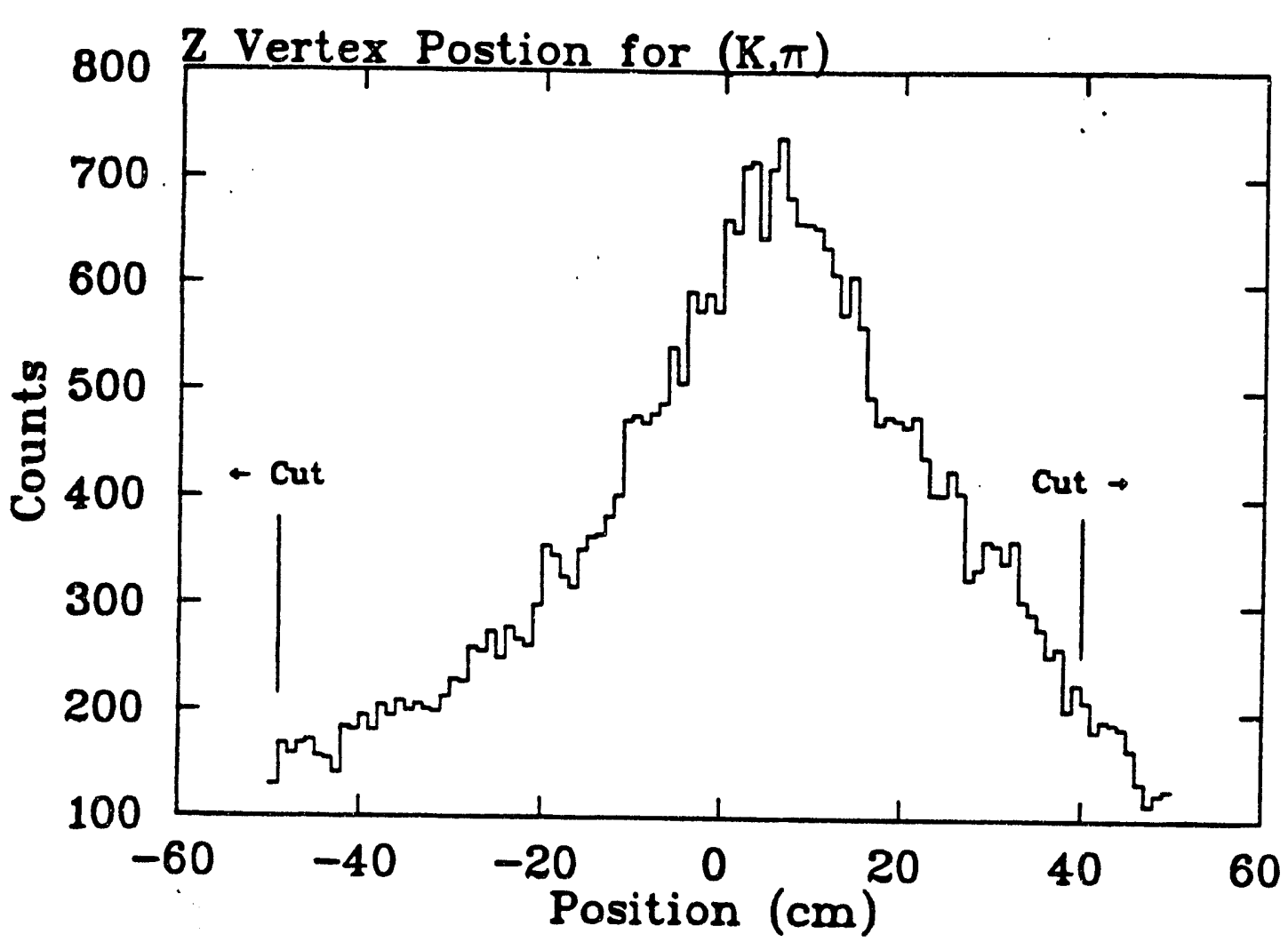

Figure 3.4 The vertex $z$ position for a subset of the raw $\left(K^{-}, \pi^{-}\right)$event sample. The vertex is determined from the position of closest approach of the upstream ray with the downstream ray. Acceptance cuts were placed at $-50 \mathrm{~cm}$ to $40 \mathrm{~cm}$.

difference of the two PMTs since this is proportional to the position along the detector element, and (2) the time average which gives the instant the detector element was hit. Thus, it makes sense to organize the calibration constants in terms of the time difference and the time average. The calibrated time average is obtained from,

$$
t_{\text {avg }}=\frac{1}{2} C\left(T D C_{A}+T D C_{B}\right)-t_{0}+\delta_{\text {svg }},
$$

where $C$ is the TDC bin-to-time conversion factor, $T D C_{A}$ and $T D C_{B}$ are raw TDC information obtained from the photomultiplier tubes at each end, respectively, $\delta_{\text {avg }}$ is the calibration constant to be determined, and $t_{0}$ is the time-zero reference time ( the time obtained from the ST2 counter ). Likewise, an expression for the time 
difference is given by,

$$
t_{d i f}=C\left(T D C_{A}-T D C_{B}\right)+\delta_{d i f},
$$

where $\delta_{\text {dif }}$ is the calibration constant to be determined. The TDC bin to time conversion factor $C$ was measured in the lab at Carnegie Mellon. The measurement setup consisted of a computer controlled precision time interval generator (Phillips 7194) which sent a stop and start pulse to each TDC channel. The TDC data was then ported to a personal computer for further analysis ( see figure 3.5 ). The motivation of these tests was to investigate full range and differential linearity. Data were collected in 1 ns intervals over the most of the 100ns range of the TDC. The time delay generated versus the TDC bin is plotted in figure 3.6 for the TDC channels used for the ST readout. Also displayed in figure 3.6 is the residual of a fit of the form $t=C(T D C)+D$ versus time delay generated. The value for $C$ for all TDC channels is equal to $25.0 \mathrm{ps} / \mathrm{bin}$ within errors. Therefore, the value $C=25 \mathrm{ps}$ is used for every channel.

Every element of the out-of-beam detector array has an optical fiber connection to the Nitrogen UV pulsed laser. A short pulse of tens of picoseconds in duration applied to the midpoint of the scintillator slab will induce the production of flourescence light. The laser pulsing system was to monitor time drifts in the light readout of the individual detectors. However, abnormally high crosstalk in the TDC's rendered the pulsed laser information useless.

An alternative time alignment is achieved from the prompt photons from the reaction

$$
\begin{aligned}
K^{-} \longrightarrow \pi^{-} & \pi^{\circ} \\
& \hookrightarrow \gamma \gamma .
\end{aligned}
$$

This decay reaction mimics the $\left(\mathrm{K}^{-}, \pi^{-}\right)$trigger because the $\pi^{-}$from the $\mathrm{K}^{-}$decay

$=$ is accepted into the downstream spectrometer. The residual $\pi^{\circ}$ is produced with a kinetic energy of about $1-4 \mathrm{MeV}$ and can be considered produced at rest. The $\pi^{\circ}$ decays to two photons $98.8 \%$ of the time with a mean life of $8.4 \times 10^{-17} \mathrm{~s}[2]$. Since our $\left(K^{-}, \dot{\pi}^{-}\right)$-neutral trigger requires a hit in the out-of-beam detector array in the absence of a SL/R-L/RK coincidence but in coincidence with the $\left(K^{-}, \pi^{-}\right)$tag, we have adequate data to make a reasonable time calibration. 


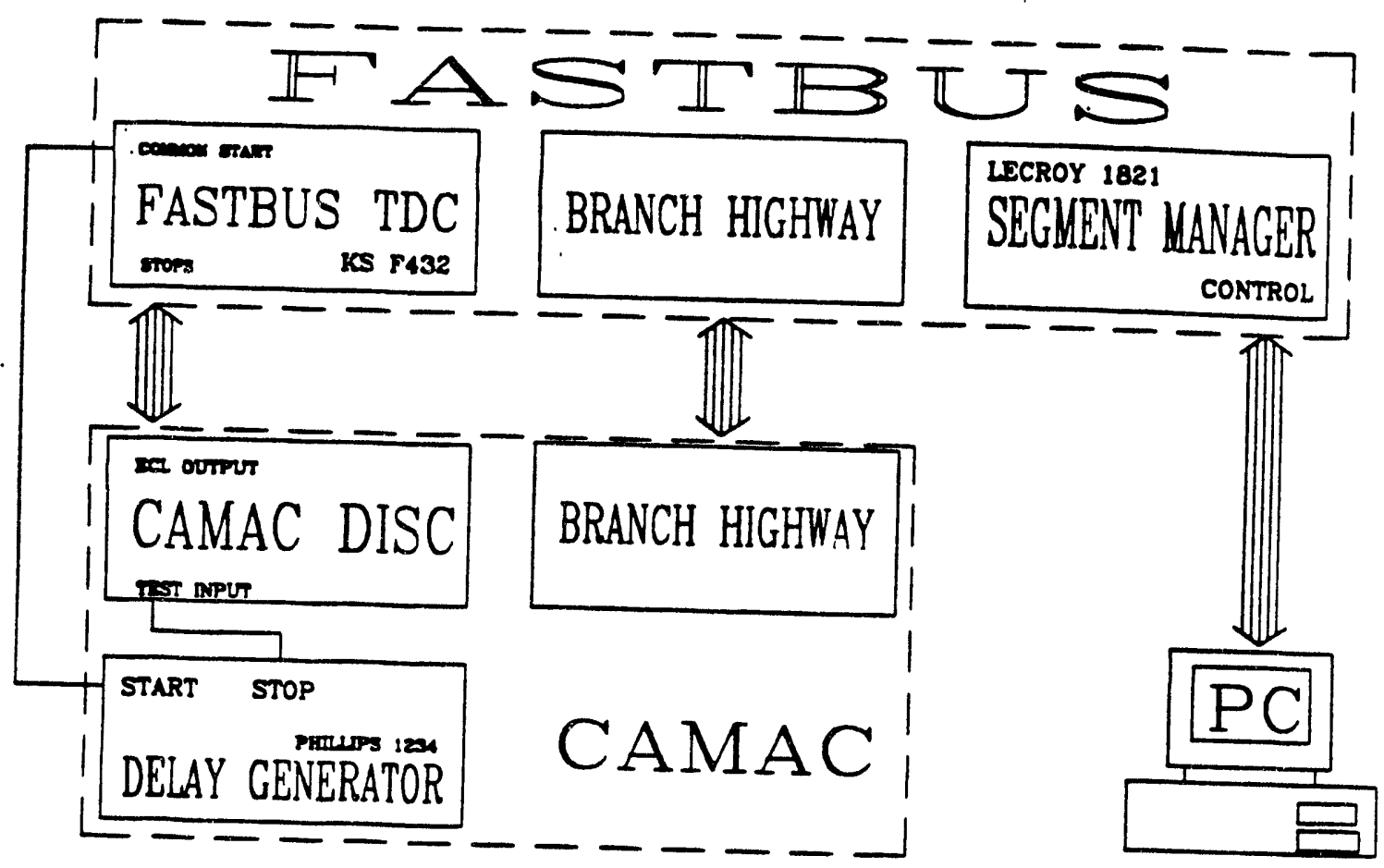

Figure 3.5 Test setup to verify internal calibration of KS 432 fastbus TDCs. A precision pulse interval generator creates start and stop pulses for the TDC. The time interval and readout is controlled by a personal computer which is interfaced to the fastbus segment manager. CAMAC control is achieved by a fastbus-CAMAC branch highway driver.

To measure the velocity of neutral particles the time interval between two points of the particles trajectory must be determined. We have information of the vertex position from the in-beam $\left(K^{-}, \pi^{-}\right)$reaction. A straight line between this vertex and the hit out-of-beam detector element is used as an estimate of the neutral particle's trajectory. The ingredients to determine the vertex time are; the mean time of ST2 $t_{a v g}^{S T 2}$, the distance between ST2 to the vertex $d_{v t s}^{S T 2}$, the incident particle's momentum $p_{i}$, and the incident particle's mass $m_{i}$. The incident particle's velocity is given by

$$
v_{i}=\frac{p_{i} c}{E_{i}}=\frac{p_{i} c}{\sqrt{p_{i}^{2} c^{2}+m_{i}^{2} c^{4}}}
$$



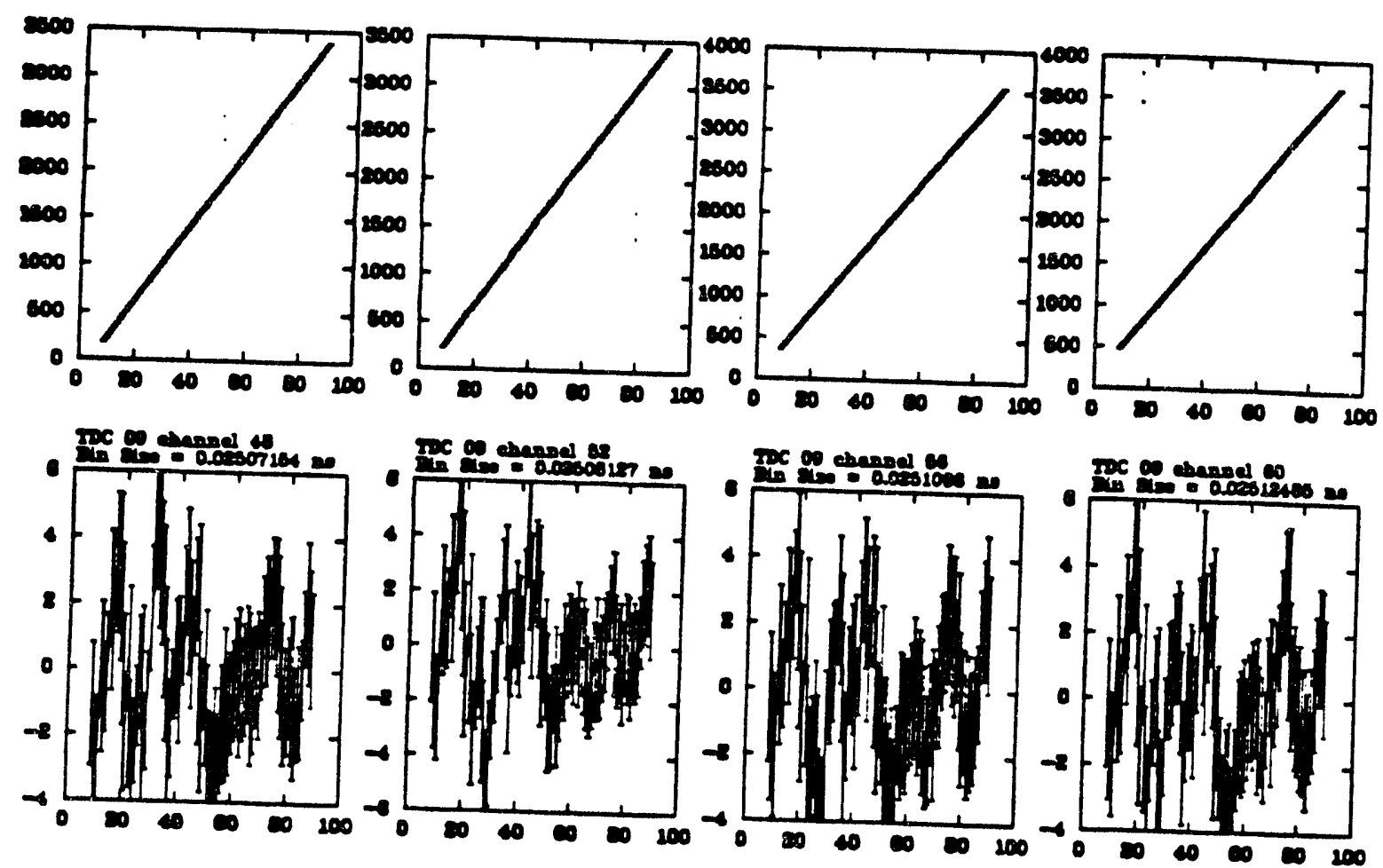

Figure 3.6 Calibration curves and residues from a straight line fit for 4 channels of the KS 432 TDC. For the top row of histograms the TDC bin number is plotted as a function of the delay time between the start and stop pulses. The lower 4 histograms are the residuals of a fit of the type $T D C=C(\Delta t)+D$. The frur channels chosen are those used on ST1 and ST2 $A$ and $B$ signals.

where $E_{i}$ is the incident particle's energy. The time at the vertex position is then

$$
t_{v t x}=\frac{d_{v t x}^{S T 2}}{v_{i}}+t_{a v g}^{S T 2}
$$

- If the mean time of the hit in the out-of-beam detector array is given by $t_{\text {avg }}^{\infty}$ and its distance to the vertex is $\alpha^{\text {ob }}$ the velocity fraction $\beta=\frac{v}{c}$ can be calculated by

$$
\beta=\frac{d^{o b}}{c\left(i_{a v g}^{o b}-t_{v t x}\right)}
$$

Figure 3.7 shows the inverse of $\beta$ ( a normalized time-of-flight ) $\beta^{-1}=\frac{c\left(t_{\text {avg }}-t_{\text {ots }}\right)}{d \phi b}$ for 

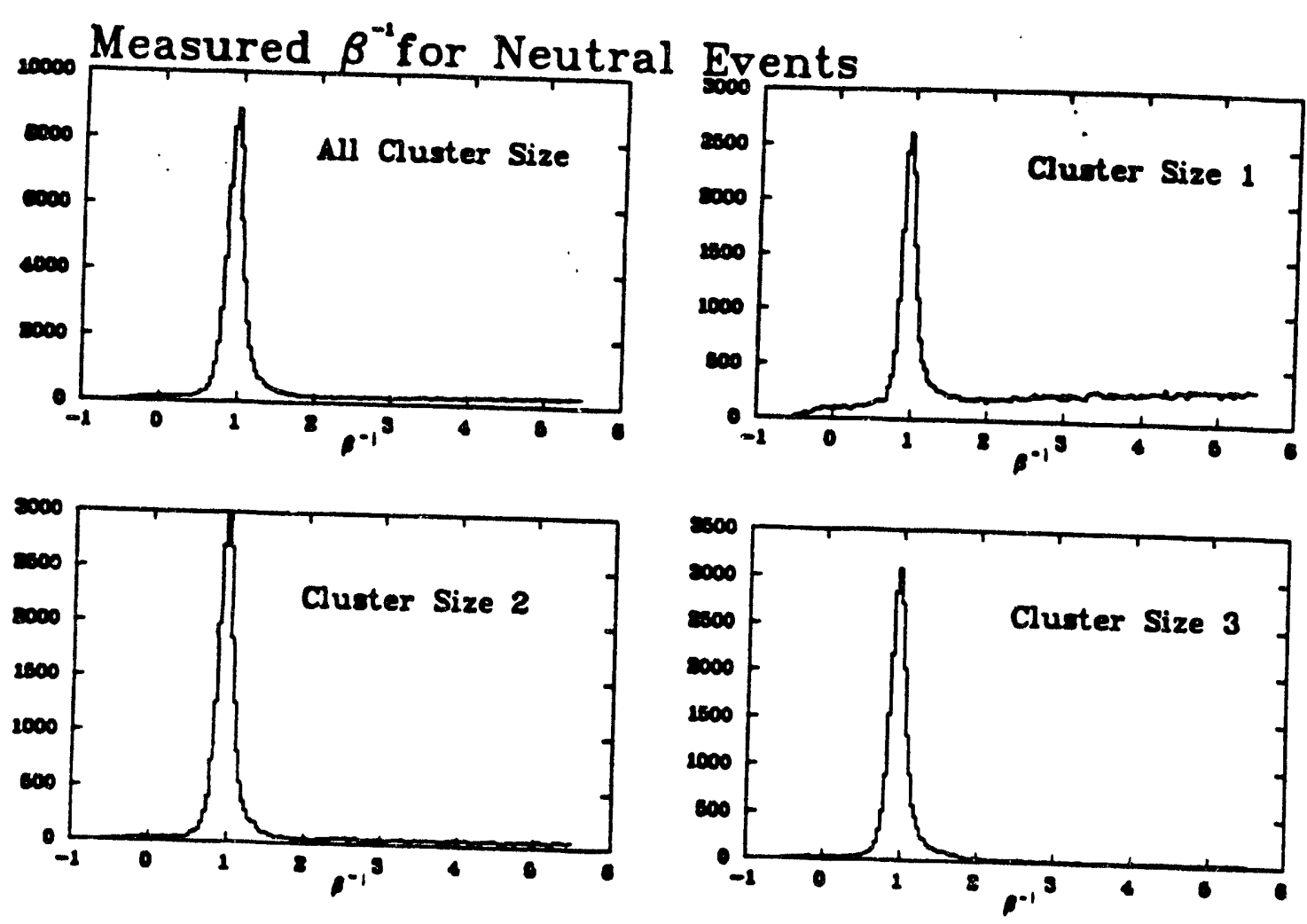

Figure 3.7 $\beta^{-1}$ is plotted with the requirement of a $\left(K^{-}, \pi^{-}\right)$trigger in coincidence with a hit in the out-of-beam detector array and the absence of a $\mathrm{SL} / \mathrm{R}-\mathrm{L} / \mathrm{RK}$ coincidence. It is evident that there is a strong dependence in the shape of the distribution for different cluster sizes found in the event.

${ }_{\Lambda}^{1}$ He $\left(K^{-}, \pi^{-}\right)$neutral sample. An abundant number of $\gamma^{\prime}$ s appear in the $\beta^{-1}=1$ peak.

Photons are distinguished from neutrons or random hits by a cluster of hits of size greater than 2 projecting toward the back of the out-of-beam array. This is evident from the series of histograms in figure 3.7 where the $\beta^{-1}=1$ peak becomes more distinguished from the flit background as the cluster size increases.

To summarize all the preceding discussion, we have all the necessary ingredients to make a mean, time calibration of the out-of-beam detector array. We have:

- a data sample of events for which we know the velocity of the particle - photons.

- the means of measuring the photon's velocity with our detector apparatus. 

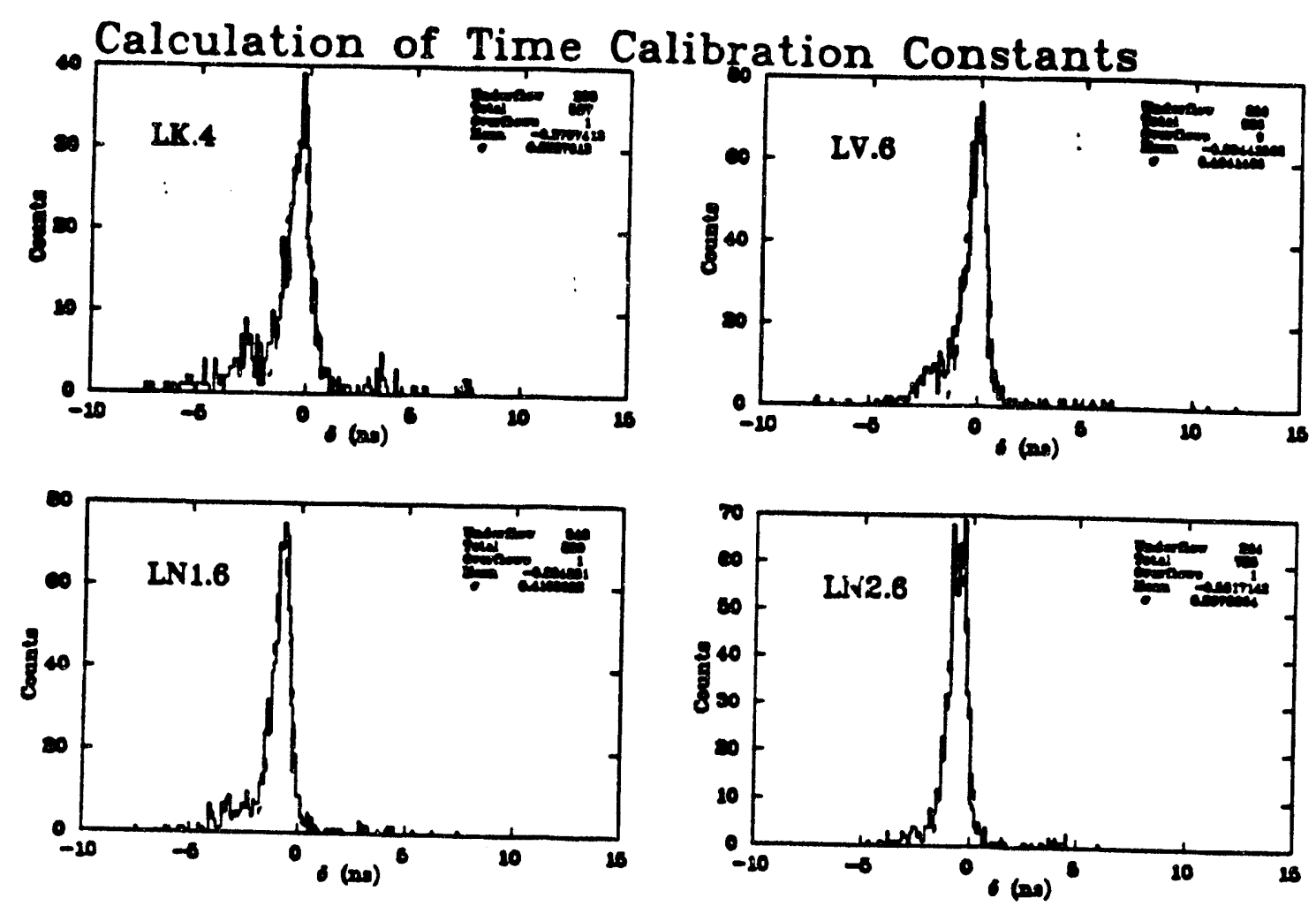

Figure 3.8 The measurement of a few mean time calibration constants $\delta_{\text {avg }}$ from equation (3.9) using neutral events in the out-of-beam detector array in coincidence with $\left(K^{-}, \pi^{-}\right)$tag. The constants were accumulated over data runs 131 to 140 .

- plenty of events so that our calibration will not be limited by statistics.

The calibration can proceed by direct measurement of the calibration constants $\delta_{\text {avg }}$ by selecting out photons and requiring $\beta^{-1}=1$, which results in,

$$
\delta_{a v g}=\frac{d^{d b}}{c}-\left(t_{a v g}^{o b}-t_{v t x}\right) \text {. }
$$

To reduce any time dependent drift, a set of calibration constants $\delta_{\text {avg }}$ is measured at intervals of ten runs. An example of distributions of $\delta_{\text {avg }}$ for a few detectors in a limited period of time is given in figure 3.8 .

The remaining time calibration constant $\delta_{\text {dif }}$ is determined from the expected distribution of charged particles upon a detector element. For a point source of charged 
particles the flux will drop off as $\frac{1}{r^{2}}$. Therefore, the charged particle distribution will peak at the point on the plane closest to the particle source: We define the time $t_{\text {dif }}=0$ to be associated with the middle of the detector element (which is extremely close to $y=0)$. Then $\delta_{\text {dif }}=C\left(T D C_{A}-T D C_{B}\right)_{\text {mean }}$.

Another feature can be derived from the TDC difference distribution - the speed of light in that detector element. The extent of the distribution is twice the time it takes light to propagate from one side to another.

\section{Time calibration of ST1}

The in-beam timing counter ST2 establishes time zero for all other detectors. That is, all times are measured with respect to ST2. Equivalently, $\delta_{a v g}^{S T 2} \equiv 0$. Since it is important to ascertain the formation time of the hypernuclear state, another counter identical to ST1 was placed just upstream of ST2 to reduce the start time resolution by $\sqrt{2}$.

The ST1 is simply calibrated by using any beam track which passes through both ST1 and ST2 which also has good tracking information from the in-beam drift chambers. The velocity is determined from equation (3.6) so that the time interval can be predicted between ST1 and ST2. The calibration constant $\delta_{\text {avg }}$ is adjusted to enforce alignment with the predicted time interval;

$$
\delta_{a v g}^{S T 1}=\frac{d_{S T}}{v_{i}}-\frac{C}{2}\left(T D C_{A}+T D C_{B}\right)
$$

where $d_{S T}$ is the distance from the particle's trajectory between ST1 and ST2. The measurement of $\delta_{a v g}$ is illustrated in figure 3.9. The timing resolution for each of the counters (assuming they behave identically) can be inferred by this distribution. The time resolution of each of the ST counters is

$$
\sigma_{S T} \leq \frac{1}{\sqrt{2}} \sigma_{\delta}=62.6 p s .
$$

\subsubsection{Energy Calibration}

Each photonultiplier tube in the experiment is connected to an Analog to Digital Converter (ADC) to measure light production from particle interactions in the detector element. The goal of calibration is to relate the ADC signal to the physical 


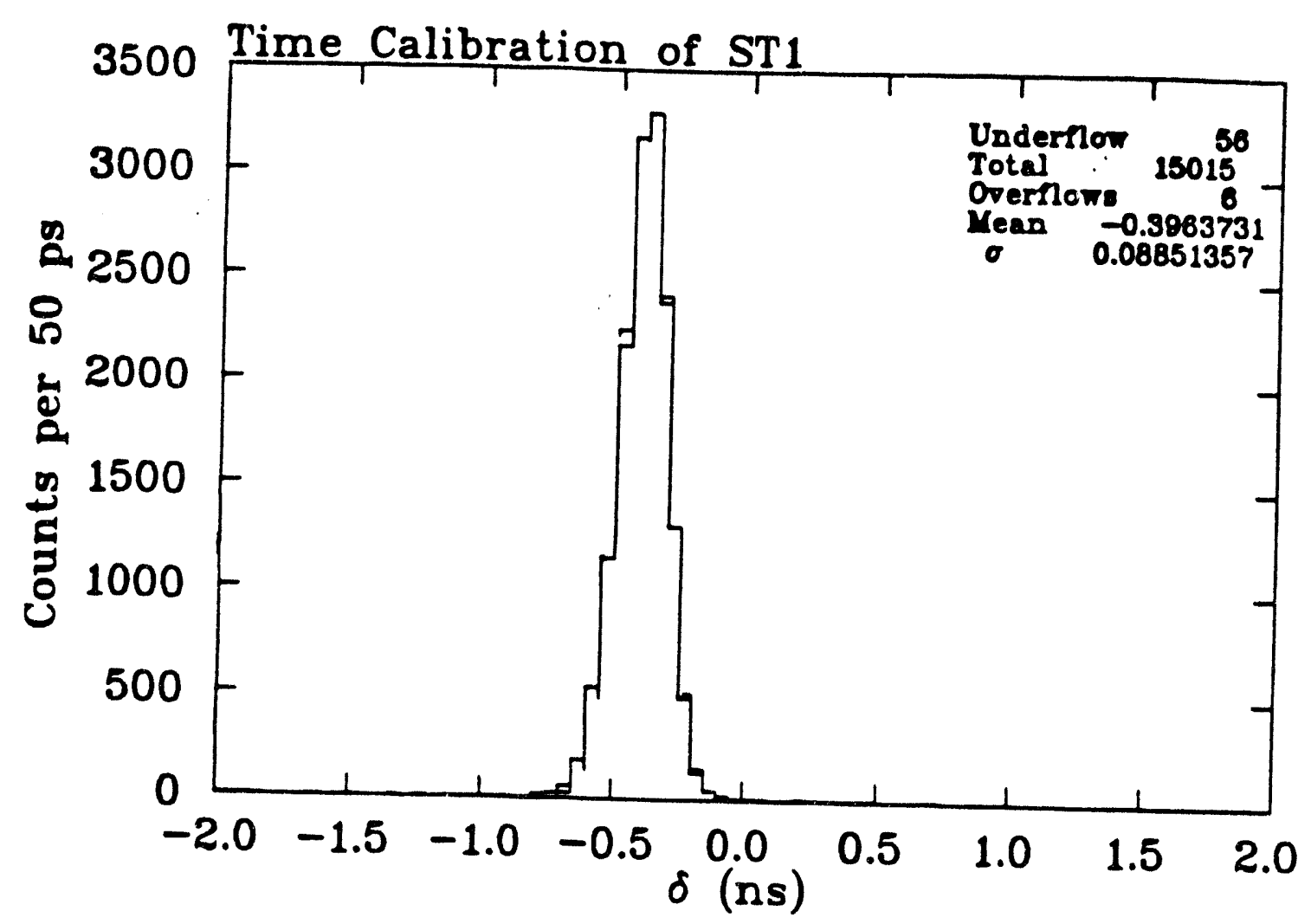

Figure 3.0 The quantity $\delta_{\text {aug }}^{S T 1}$ from equation (3.10) is plotted for identified $K^{-}$incident upon the ${ }^{4} \mathrm{He}$ target.

quantity of energy deposited into the detector element. The heart of the calibration procedure is a comparison of the theoretical behaviour of the detectors to the ADC response.

The response of charged particle interactions with organic scintillator used in particle detection is well modeled. We use the program ELOSS developed at University of Washington by Hans Bichsel which is based upon the Bethe-Block equations of energy loss and range (with $Z^{3}$ and shell corrections). The input to the program $=$ includes (1) the incident particle type and mass, (2) energy range, and (3) the chemical composition of the material. The program produces coefficients $a$ and $c$ for the approximate energy losis $\frac{d E}{d x}=c E^{a}$ where $E$ is the kinetic energy of the interacting particle.

The light produced in scintillator and measured by photomultiplier tubes through ADC's is a function of the energy deposited. Unfortunately, the scintillator is not 
completely transparent to the produced scintillation light. The attenuation is assumed to behave as

$$
I(x)=I_{0} e^{-x / \lambda}
$$

where $\lambda$ is the attenuation length ( $\lambda \approx 1 m$ for this experiment ) and $x$ is the distance from the particle interaction point to the photomultiplier tube. This adverse effect is countered by taking the geometric average of the two light measurements

$$
\begin{aligned}
I_{g}(x) & =\sqrt{I(x) I(l-x)} \\
& =I_{0} \sqrt{e^{-l / \lambda}}
\end{aligned}
$$

which is independent of $x$ ( $l$ is the length of the detector element).

It is assumed that the calibrated energy is linearly proportional to the ADC count $E=\alpha(A D C-P)$ where $\alpha$ is the ADC to energy conversion factor and $P$ is the pedestal offset. The ADCs used in this experiment employ an automatic pedestal subtraction, which leaves only one parameter, $\alpha$, to determine for the calibration. The calibrated energy from the geometric mean of the ADCs is then

$$
E=\alpha^{\prime} \sqrt{A D C_{A} \times A D C_{B}}
$$

where the calibration constant $\alpha^{\prime}$ is a redefined as the geometric mean of the single ADC constants.

To carry out the calibration it is necessary to isolate an event sample for which the particle identification relies only upon the velocity measurement. The event sample chosen is the scattering of $\pi^{-}$from ${ }^{4} \mathrm{He}$.

$$
\pi^{-}+{ }^{4} \mathrm{He} \rightarrow \pi^{-}+X
$$

The behavior of the scattered $\pi^{-}$is predicted from basic kinematics. and is fairly independent of the scattering angle.

The calibration constants for each detector are directly measured by making Gaussian fits to the bistograms of

$$
\alpha^{\prime}=\frac{E_{p r e d}}{\sqrt{A D C_{A} \times A D C_{B}}}
$$

where $E_{p r e d}$ is determined from the measured velocity and the above relation for $\frac{d E}{d x}$. 


\subsection{Decay Product Analysis}

The design goals of the out-of-beam detector arrays include charged particle identification and a capacity to determine the particle's energy.

\subsubsection{Out-of-Beam Tracking}

A reliable tracking algorithm in the out-of-beam detectors is key for proper particle identification. A tracking code must consistently select valid tracks out of the many possible permutations of hit combinations. Also accurate trajectories are required for good time-of-flight measurements. Charged trajectories provide much more information than neutral ones and are thus handled somewhat differently.

To find a valid out-of-beam charged track, we first consider all permutations of good hits between the SL (SR) and LK (RK) layers. A good hit is one which satisfies the following:

- Both PMT signals are above threshold and trigger the TDC digitization.

- The $y$ hit position is within the scintillator's dimension to within the position resolution of the detector. The TDC difference is used to determine the position of the hit along the scintillator.

- The calibrated energy for the detector hit is above a selected value.

We next remove ambiguities for hits in the out-of-beam chambers by including the hit into our trajectory calculation which yields the best linear fit. Using only the information gathered so far, a rough estimate is made for the trajectory by making a least squares fit. Now that we have a clue as to the direction of the particle's trajectory we can evaluate whether other out-of-beam hits should be included in a more refined trajectory calculation. We examine all other good hits and require the hit under consideration to be within a selected tolerance which is proportional to the detector dimensions. A linear least squares fit is then made and various quantities such as $\chi^{2}$, time-of-flight, range, and $d E / d x$ for the second to last layer are calculated. Once all SL-LK (SR-RK) combinations are exhausted, the tracks are sorted according to their normalized $\chi^{2}$ and stored into the data base (SYNOP) for further analysis. 
A flow chart illustrating the charged tracking algorithm, QTRACK, is given in figure 3.10 .

To make a time-of-flight measurement of neutral decay products we need the time difference between the hypernuclear decay and hit in the neutron detector array. We use the ST time measurement along with time-to-vertex corrections to determine the hypernuclear formation time. The ST counter is used as the time reference for all other detector elements in the experiment. The vertex of the $\left(\mathrm{K}^{-}, \pi^{-}\right)$reaction is determined from the closest approach of the up-stream spectrometer track with the down-stream spectrometer track. There is a fairly large uncertainty associated with the $z$-vertex position for the ${ }_{\Lambda}^{4} \mathrm{He}$ experiment since we were interested in $\left(\mathrm{K}^{-}, \pi^{-}\right)$ scattering angles close to zero degrees. Analysis of the upstream spectrometer track also gives the momentum of the $\mathrm{K}^{-}$, and thus its velocity. The time at the vertex can now be determined by $t_{0}=\frac{\left|x_{\text {veries }}-x_{S T}\right|}{v_{K}}$. We search for good hits and/or clusters of good hits in the neutron detector arrays. A cluster is defined as a set of contiguous hits in the neutron detector which is isolated from hits in the $x-z$ plane and separated by at least $50 \mathrm{~cm}$ in the $y$ direction. Once a cluster is found, the LV (RV) and LK (RK) are checked for hits which would be consistent with the cluster. If there are aligned hits between the cluster and these first layers of the out-of-beam array then the track is classified as a charged track which failed to trigger an SL (SR) (the charged track may have passed through a crack in the SL/R array). A least squares fit is made to the valid neutral cluster. The time-of-flight is determined from the element in the cluster closest to the vertex and earliest in time. Once all clusters are found, the tracks are then stored into the SYNOP data base. A flow chart illustrating the neutral tracking algorithm, NTRACK, is given in figure 3.11 .

\subsubsection{Charged Particle Identification}

The main challenge of charged particle identification is separation of protons and pions. The challenge stems from the lack of an external momentum analyzing magnetic field. However, there are a few characteristics of the charged track which facilitate the identification:

- velocity The charged particle's velocity is measured by time-of-flight from the target to the out-of-beam detectors. For protons with kinetic energies from 20 to $150 \mathrm{MeV}$ we would expect velocity fractions between $\beta_{p}=0.20 \rightarrow 0.51$. 


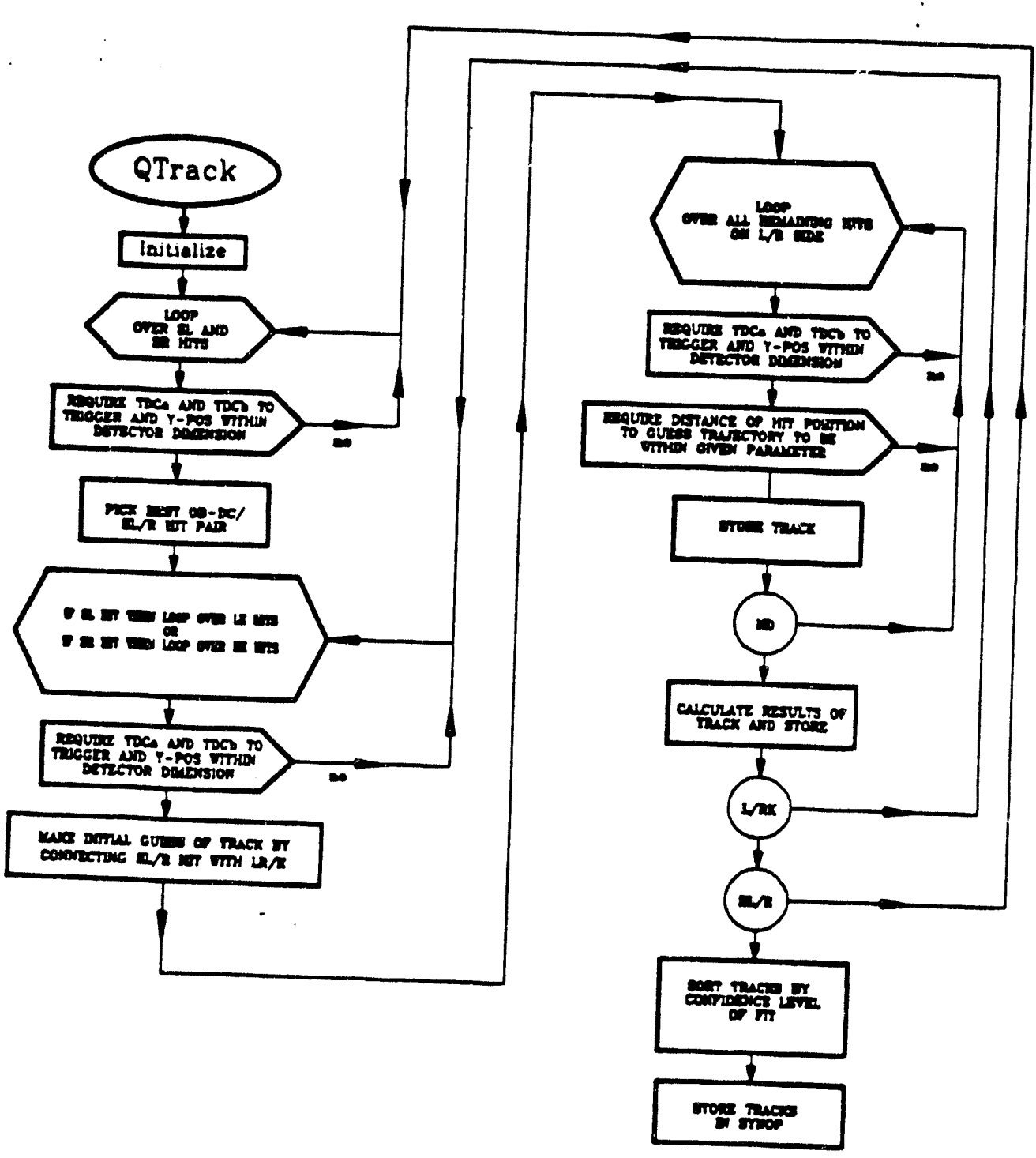

Figure 3.10 Tracking algorithm for charged tracks in the out-of-beam detector arrays. A charge trigger is defined as a coincidence of bits in the (SL and LK) or (SR and RK). 


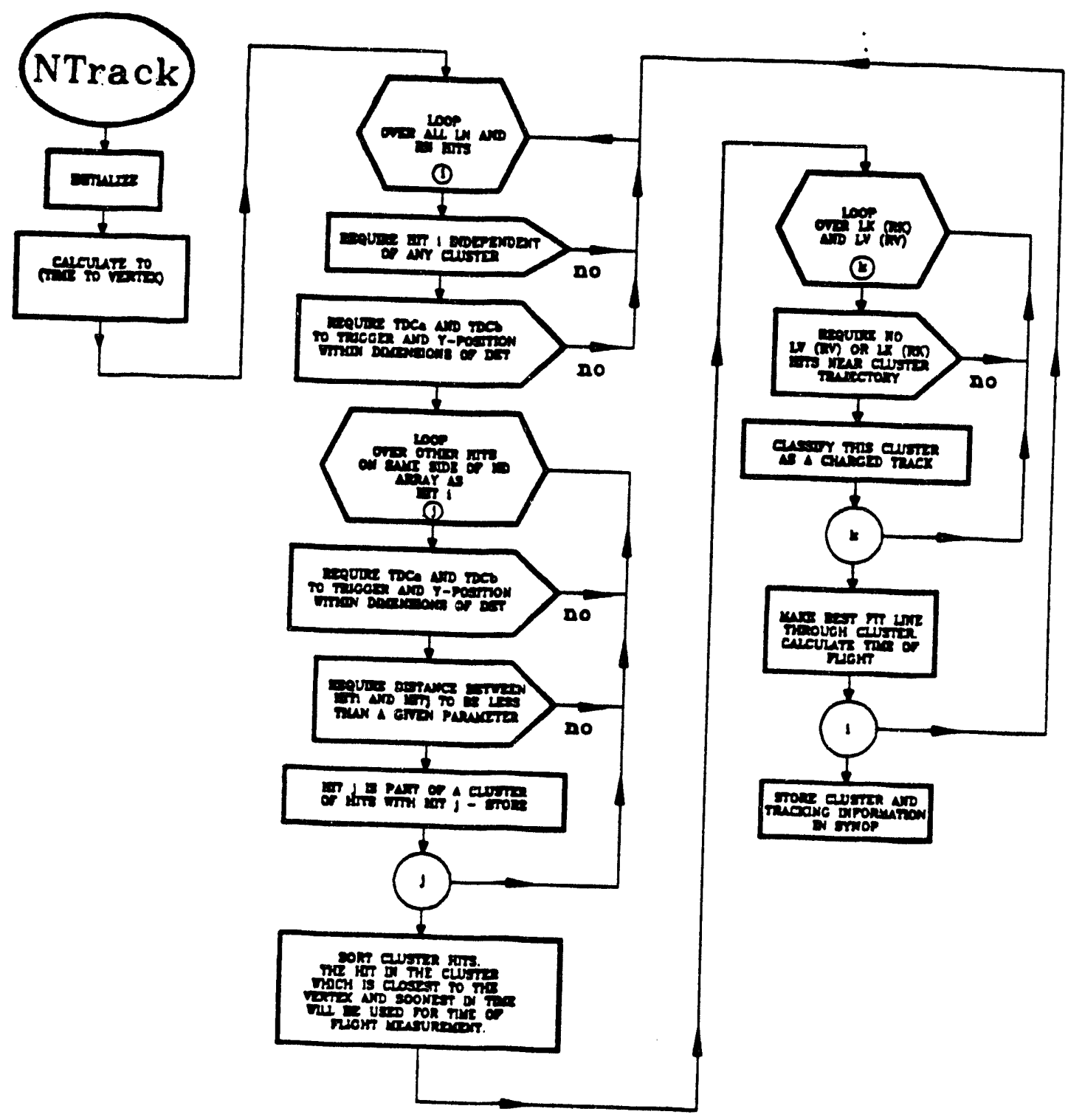

Figure 3.11 Neutral particle tracking algorithm in the out-of-beam detector arrays. A neutral trigger is defined as a hit or a cluster of hits in the neutron detector array with an absence of hits in the neutron veto arrays, $\mathrm{LV}$ and $\mathrm{RV}$, for leit and right side hits, respectively. 
Likewise, for pions of 10 to $40 \mathrm{MeV}$ would have a $\beta_{\pi}=0.36 \rightarrow 0.63$. Since there is an overlap, a velocity measurement is not sufficient to establish particle identification.

- range The pions will range out in a shorter distance than protons with similar velocities. For example, a $\beta=0.4$ proton will lose all of its kinetic energy to material interactions in scintillator after about $5 \mathrm{~cm}$. A $\beta=0.4$ pion will stop in about $1.2 \mathrm{~cm}$. The range cannot be determined much better than the thickness of our out-of-beam detector elements since we have no way of knowing the stop position within the detector element.

- energy loss Protons lose energy at a higher rate than pions with similar kinetic energies as they traverse a material. Equivalently, protons have a larger (more negative) $\frac{d E}{d x}$. This can be illustrated by comparing a $30 \mathrm{MeV}$ proton and pion. The proton will lose energy at a rate about 4 times that of a pion in scintillator. A major drawback to this approach is that as a $\pi^{-}$comes to rest in a material it can be captured in a nuclear orbit and result in an unpredictable nuclear interaction which deposits an indefinite amount of energy into the detector element. This is referred to as pion starring. This can be circumvented if we limit ourselves to measurement of the energy loss for oniy those detector elements which the particle traverses completely.

The best possible method of particle identification is obtained from a combination of these methods.

Tracks are separated by range. Charge particle events are classified according to the stopping layer. For events which penetrate the second layer of the out-of-beam detector array, layer $\mathrm{L} / \mathrm{RK}$, the response of the $\mathrm{L} / \mathrm{RK}$ layer is used to make particle identification. For those events which stop in the L/RK layer, the response of the

- SL/R layer is used to make the particle identification.

The detector response is predictable for a given species of interacting particles (see section 3.3.2). The energy loss in $\mathrm{L} / \mathrm{RK}$ and velocity information are combined to compare with the theoretical behavior of the charged track. An identification decision is made based upon the proximity of the particle to the pion/proton theoretical curves in the $\beta / \Delta E$ plane. 
- Energy loss is calculated for charged tracks emitted from the target. This is subdivided into losses in the liquid Helium vessel and losses in the PVC shell.

- Straggling is included into the energy loss calculation for the charged particles $\frac{d E}{d x}$ calculations.

- A minimum energy deposition per detector element is required to emulate hardware threshold levels.

- The measured hit time is smeared to account for the finite time resolution for each detector element type. A value is extracted from a random Gaussian distribution with a $\sigma$ estimated from measured detector resolution. This value is then added to the calculated hit time.

- The measured energy deposition is smeared to reflect the finite energy resolution of each detector element. A value selected from a Gaussian distribution with a width proportional to the calculated energy deposition is added to the calculated energy deposition. The proportionality constant used is 0.30 and was determined by comparison with actual energy deposition spectra.

- The probability of a detectable neutron interaction in a given medium is dependent upon the energy of the neutron, the medium composition, and the detector energy threshold limit. For a given threshold, the probability of interaction is given by a Monte Carlo of neutron interactions created at Kent State and modified specifically for our detector by Bernd Bassalleck[20].

For protons (pions), $G=5000$ events are generated for every kinetic energy bin of $5 \mathrm{MeV}$ from $5 \mathrm{MeV}$ to $175 \mathrm{MeV}(5 \mathrm{MeV}$ to $100 \mathrm{MeV}) . G_{n}=25000$ neutrons are generated and binned the same as the protons. These generated Monte Carlo events are processed in the same manner as real hypernuclear decay data.

If the Monte Carlo produces events that are a good representation of the real data, the particle composition for each identified sample in kinetic energy bin $E$ is then related to Monte Carlo by

$$
D_{p}\left(E_{p}\right)=\frac{N_{p p}\left(E_{p}\right)}{G} N_{p}\left(E_{p}\right)+\frac{N_{\pi p}\left(E_{p}\right)}{G} N_{\pi}\left(E_{p}\right)
$$


fitting the excitation distribution to the expected one. The total number is found by integrating the fit. We will call this sum $N_{i}^{e e}$, where $i$ stands for proton, pion, or neutron.

The next step is to isolate a representative sub-sample of $N_{i}^{e e}$ events which are known to be from hypernuclear decay. This is done by placing a tight cut upon the excitation energy spectrum at the hypernuclear excitation energy peak. The total number of events in this sub-sample is $N_{i}^{2 \sigma}$. A correction for this restricting cut is made towards the end of the acceptance correction procedure.

Once we have constructed the $N_{i}^{2 \sigma}$ event sample, we can then determine the kinetic energy distribution. This distribution is organized into $5 \mathrm{MeV}$ bins. The number of events in bin $E$ is $D_{i}(E)$.

At this point, we must make the acceptance correction. We must make allowances for the deficiencies and inefficiencies of the total detector system. These inefficiencies can be estimated by modeling the performance of the detector in a computer simulation. In such a model, particle trajectories are generated according to prescribed distributions and are tracked through the detector system. This technique of determining the detector acceptance and efficiency is formally called Monte Carlo. The computer model for this experiment was developed in collaboration with Valdis Zeps.

The generated trajectory, particle type, and initial energy limits (from a flat distribution) are input into the program. This means that a study of the detector's response to a detailed energy sweep for a given particle type can be studied. Some important features of the Monte Carlo include:

- Events are generated to provide a uniform solid angle coverage. A flat distribution is generated for $\phi$ and $\cos \theta$ where $\phi$ and $\theta$ are the polar and axial angles, respectively.

- The distribution of the source position is the shape of the $K^{-}$incident beam in $x$ and $y$ and is confined to $-10 \mathrm{~cm}$ to $20 \mathrm{~cm}$ in $z$ (the $z$ extent of the ${ }^{4} \mathrm{He}$ target).

- Multiple scattering is included for charged tracks interacting with the detector elements and target.

- For pion studies, the decay $\pi^{-} \longrightarrow \mu^{-}+\overline{\nu_{\mu}}$ is included. 
- Energy loss is calculated for charged tracks emitted from the target. This is subdivided into losses in the liquid Helium vessel and losses in the PVC shell.

- Straggling is included into the energy loss calculation for the charged particles $\frac{d E}{d x}$ calculations.

- A minimum energy deposition per detector element is required to emulate hardware threshold levels.

- The measured hit time is smeared to account for the finite time resolution for each detector element type. A value is extracted from a random Gaussian distribution with a $\sigma$ estimated from measured detector resolution. This value is then added to the calculated hit time.

- The measured energy deposition is smeared to reflect the finite energy resolution of each detector element. A value selected from a Gaussian distribution with a width proportional to the calculated energy deposition is added to the calculated energy deposition. The proportionality constant used is 0.30 and was determined by comparison with actual energy deposition spectra.

- The probability of a detectable neutron interaction in a given medium is dependent upon the energy of the neutron, the medium composition, and the detector energy threshold limit. For a given threshold, the probability of interaction is given by a Monte Carlo of neutron interactions created at Kent State and modified specifically for our detector by Bernd Basselleck[20].

For protons (pions), $G=5000$ events are generated for every kinetic energy bin of $5 \mathrm{MeV}$ from $5 \mathrm{MeV}$ to $175 \mathrm{MeV}(5 \mathrm{MeV}$ to $100 \mathrm{MeV}) . G_{n}=25000$ neutrons are generated and binned the same as the protons. These generated Monte Carlo events are processed in the same manner as real hypernuclear decay data.

If the Monte Carlo produces events that are a good representation of the real data, the particle composition for each identified sample in kinetic energy bin $E$ is then related to Monie Cario by

$$
D_{p}\left(E_{p}\right)=\frac{N_{p p}\left(E_{p}\right)}{G} N_{p}\left(E_{p}\right)+\frac{N_{\pi p}\left(E_{p}\right)}{G} N_{\pi}\left(E_{p}\right)
$$




$$
\begin{aligned}
& D_{\pi}\left(E_{\pi}\right)=\frac{N_{p \pi}\left(E_{\pi}\right)}{G} N_{p}\left(E_{\pi}\right)+\frac{N_{\pi \pi}\left(E_{\pi}\right)}{G} N_{\pi}(E) \\
& D_{n}(E)=\frac{N_{p n}(E)}{G} N_{p}(E)+\frac{N_{\pi n}(E)}{G} N_{\pi}(E)+\frac{N_{n N}(E)}{G_{n}} N_{n}(E)
\end{aligned}
$$

where $N_{i j}(E)$ are the number of generated events of particle type $i$ which were identified as particle type $j$ at energy $E_{i}$. The ratios, $N_{i j}(E) / G$ are the Monte Carlo acceptance correction factors. That is, after generating $G$ events of particle type $i$ in energy bin $E$, the analysis code identified $N_{i j}(E)$ events. The numbers which we wish to extract are the number of actual particles of type $i$ at energy $E ; N_{i}(E)$. The equations (3.15) are inverted and solved for the $N_{i}(E)$ 's. The quantities $N_{i j}(E)$ are summarized in tables $3.1,3.2$, and 3.3.

The kinetic energy shape is modeled so that predictions can be made beyond the acceptance of the detector system. From the model, the number of acceptance corrected events is $M_{i}(E)$. The total number of events in the kinetic energy distribution for particle type $i$ is obtained from summing the modeled events, $\sum_{E} M_{i}(E)$.

The last step in the acceptance correction procedure is to make allowance for the tight excitation energy cut made earlier on. As mentioned before, $N_{i}^{20}$ is the number of events remaining after the tight excitation energy cut. $N_{i}^{e e}$ is the number of observed events as determined from a fit to the excitation energy shape. To undo the excitation energy cut, the total number of events are multiplied by a factor of $N_{i}^{e e} / N_{i}^{2 \sigma}$. That is,

$$
N_{i}^{\text {tot }}=\frac{N_{i}^{e e}}{N_{i}^{20}} \sum_{E} M_{i}(E)
$$

This description of the correction procedure may appear rather abstract upon first review. The procedure is applied for the actual proton, pion, and neutron measurement in the following chapter.

\section{= 3.6 Target Veto Threshold Calibration}

The purpose of the "He target veto system is to reduce the source of coincident neutrons due to $\pi^{-}$abscrbtion

$$
\pi^{-}+X \longrightarrow n+X
$$




\begin{tabular}{|c|c|c|c|c||c|c|c|c|c|}
\hline \multicolumn{10}{|c|}{ Proton Acceptance Study } \\
\hline \hline$E_{0}$ & Protons & Pions & Neutrons & No ID & $E_{\circ}$ & Protons & Pions & Neutrons & No ID \\
& $N_{p p}$ & $N_{p \pi}$ & $N_{p n}$ & $N_{p u}$ & & $N_{p p}$ & $N_{p \pi}$ & $N_{p n}$ & $N_{p u}$ \\
\hline 5 & 0 & 0 & 0 & 0 & 90 & 1717 & 3 & 18 & 4 \\
10 & 0 & 0 & 0 & 0 & 95 & 1613 & 3 & 10 & 7 \\
15 & 0 & 0 & 0 & 0 & 100 & 1674 & 13 & 10 & 13 \\
20 & 0 & 1 & 0 & 0 & 105 & 1622 & 10 & 10 & 18 \\
25 & 1 & 3 & 0 & 1 & 110 & 1616 & 9 & 14 & 25 \\
30 & 91 & 12 & 0 & 2 & 115 & 1578 & 8 & 9 & 30 \\
35 & 703 & 20 & 0 & 9 & 120 & 1627 & 12 & 15 & 24 \\
40 & 1544 & 25 & 0 & 8 & 125 & 1586 & 7 & 21 & 20 \\
45 & 1774 & 22 & 0 & 19 & 130 & 1528 & 1 & 27 & 28 \\
50 & 1782 & 37 & 0 & 42 & 135 & 1513 & 5 & 22 & 37 \\
55 & 1847 & 20 & 0 & 58 & 140 & 1525 & 3 & 41 & 35 \\
60 & 1801 & 19 & 0 & 24 & 145 & 1449 & 10 & 24 & 35 \\
65 & 1777 & 18 & 6 & 9 & 150 & 1422 & 11 & 36 & 77 \\
70 & 1752 & 2 & 6 & 3 & 155 & 1435 & 2 & 40 & 105 \\
75 & 1709 & 2 & 11 & 7 & 160 & 1390 & 5 & 35 & 151 \\
80 & 1746 & 3 & 11 & 6 & 165 & 1376 & 4 & 40 & 147 \\
85 & 1673 & 5 & 12 & 9 & 170 & 1346 & 5 & 53 & 106 \\
\hline
\end{tabular}

Table 3.1 The energy dependent acceptance is studied by the analysis of events generated by a computer model of the E788 detector system. 10000 proton events are generated in each $5 \mathrm{MeV}$ bin. The energy listed in columns one and six refers to the lower edge of the bin $E_{0}$. 


\begin{tabular}{|c|c|c|c|c|}
\hline \multicolumn{5}{|c|}{ Pion Acceptance Study } \\
\hline \hline$E_{0}$ & Protons $N_{\pi p}$ & Pions $N_{\pi \pi}$ & Neutrons $N_{\pi n}$ & No ID $N_{\pi u}$ \\
\hline 5 & 0 & 1 & 0 & 1 \\
10 & 14 & 107 & 0 & 4 \\
15 & 87 & 1251 & 0 & 17 \\
20 & 26 & 1688 & 0 & 61 \\
25 & 26 & 1606 & 0 & 103 \\
30 & 3 & 1555 & 7 & 183 \\
35 & 0 & 1748 & 14 & 12 \\
40 & 0 & 1637 & 13 & 9 \\
45 & 2 & 1613 & 25 & 20 \\
50 & 2 & 1626 & 21 & 16 \\
55 & 4 & 1502 & 31 & 35 \\
60 & 5 & 1461 & 30 & 52 \\
65 & 2 & 1303 & 34 & 194 \\
70 & 1 & 1205 & 54 & 271 \\
75 & 0 & 1200 & 40 & 183 \\
\hline
\end{tabular}

Table 3.2 The energy dependent acceptance is studied by the analysis of events generated by a computer model of the E788 detector system. 10000 pion cvents are generated in each $5 \mathrm{MeV}$ bin. The energy listed in column one refers to the lower edge of the bin. 


\begin{tabular}{|c|c|c|c|c||c|c|c|c|c|}
\hline \multicolumn{8}{|c|}{ Neutron Acceptance Study } \\
\hline \multicolumn{2}{|c|}{50000 Neutron Events Generated per Bin } \\
\hline \hline$E_{0}$ & Protons & Pions & Neutrons & No ID & $E_{\circ}$ & Protons & Pions & Neutrons & No ID \\
& $N_{n p}$ & $N_{n \pi}$ & $N_{n n}$ & $N_{n u}$ & & $N_{n p}$ & $N_{n \pi}$ & $N_{n n}$ & $N_{n u}$ \\
\hline 5 & 0 & 0 & 236 & 0 & 90 & 0 & 0 & 2931 & 0 \\
10 & 0 & 0 & 1813 & 0 & 95 & 0 & 0 & 2979 & 0 \\
15 & 0 & 0 & 3081 & 0 & 100 & 0 & 0 & 2831 & 0 \\
20 & 0 & 0 & 3028 & 0 & 105 & 0 & 0 & 2759 & 0 \\
25 & 0 & 0 & 3046 & 0 & 110 & 0 & 0 & 2589 & 0 \\
35 & 0 & 0 & 3095 & 0 & 115 & 0 & 0 & 2660 & 0 \\
35 & 0 & 0 & 3238 & 0 & 120 & 0 & 0 & 2703 & 0 \\
40 & 0 & 0 & 3472 & 0 & 125 & 0 & 0 & 2558 & 0 \\
45 & 0 & 0 & 3547 & 0 & 130 & 0 & 0 & 2638 & 0 \\
50 & 0 & 0 & 3597 & 0 & 135 & 0 & 0 & 2520 & 0 \\
55 & 0 & 0 & 3563 & 0 & 140 & 0 & 0 & 2581 & 0 \\
60 & 0 & 0 & 3442 & 0 & 145 & 0 & 0 & 2691 & 0 \\
65 & 0 & 0 & 3290 & 0 & 150 & 0 & 0 & 2698 & 0 \\
70 & 0 & 0 & 3119 & 0 & 155 & 0 & 0 & 2791 & 0 \\
75 & 0 & 0 & 3061 & 0 & 160 & 0 & 0 & 2683 & 0 \\
80 & 0 & 0 & 2924 & 0 & 165 & 0 & 0 & 2680 & 0 \\
85 & 0 & 0 & 3049 & 0 & 170 & 0 & 0 & 2623 & 0 \\
\hline
\end{tabular}

Table 3.3 The energy dependent acceptance is studied by the analysis of events generated by a computer model of the E788 detector system. 50000 neutron events are generated in each $5 \mathrm{MeV}$ bin. The energy listed in columns one and six refers to the lower edge of the bin $E_{0}$. 
Typically, a $\pi^{-}$is absorbed upon a proton-neutron pair (deuteron) within a nucleus. The deuteron- $\pi^{-}$system transmutes into two energetic neutrons

$$
\pi^{-}+d \longrightarrow n+n+137 \mathrm{MeV} \text {. }
$$

These neutrons are a source of background for the stimulated decay neutron measurement.

The design goal of the cryogenic internal veto system is merely to tag charged particles emitted from the target. In principal, this is easily achieved by placing a threshold cut in the ADC distribution provided the pedestal and data are well separated. This separition is complicated for this experiment because (1) the optical attenuation in the $34 \mathrm{~cm}$ long scintillator may be large enough to cause a strong position dependence in the measured pulse beight, and (2) the photomultiplier tubes used in the target veto were susceptible to the high level of ambient $\mathrm{AC}$ noise of the AGS environment.

The first point is irrecoverable and is an admitted failure for our choice of materials used in construction. We were under a nearly impossible deadline to design, construct, and debug the E788 apparatus. There was not enough time to purchase the proper materials nor test the final result. Thus, the position dependence due to optical attenuation must be accepted.

The second point, widening of the pedestal from ambient $\mathrm{AC}$ noise, did not pose such a problem. We took measures to correlate the time dependence of the ADC signals by recording the phase of the ambient $60 \mathrm{~Hz}$ line noise. Figure 3.13 illustrates the device used to record the phase of the $60 \mathrm{~Hz}$ ambient noise. The phase dependence for the ADC measurement is shown in figure 3.14.

A phase correction to the target veto ADC's is made by aligning the pedestal for each phase bin. The ADC threshold value is placed at two sigma above the pedestal peak for each target detector. 

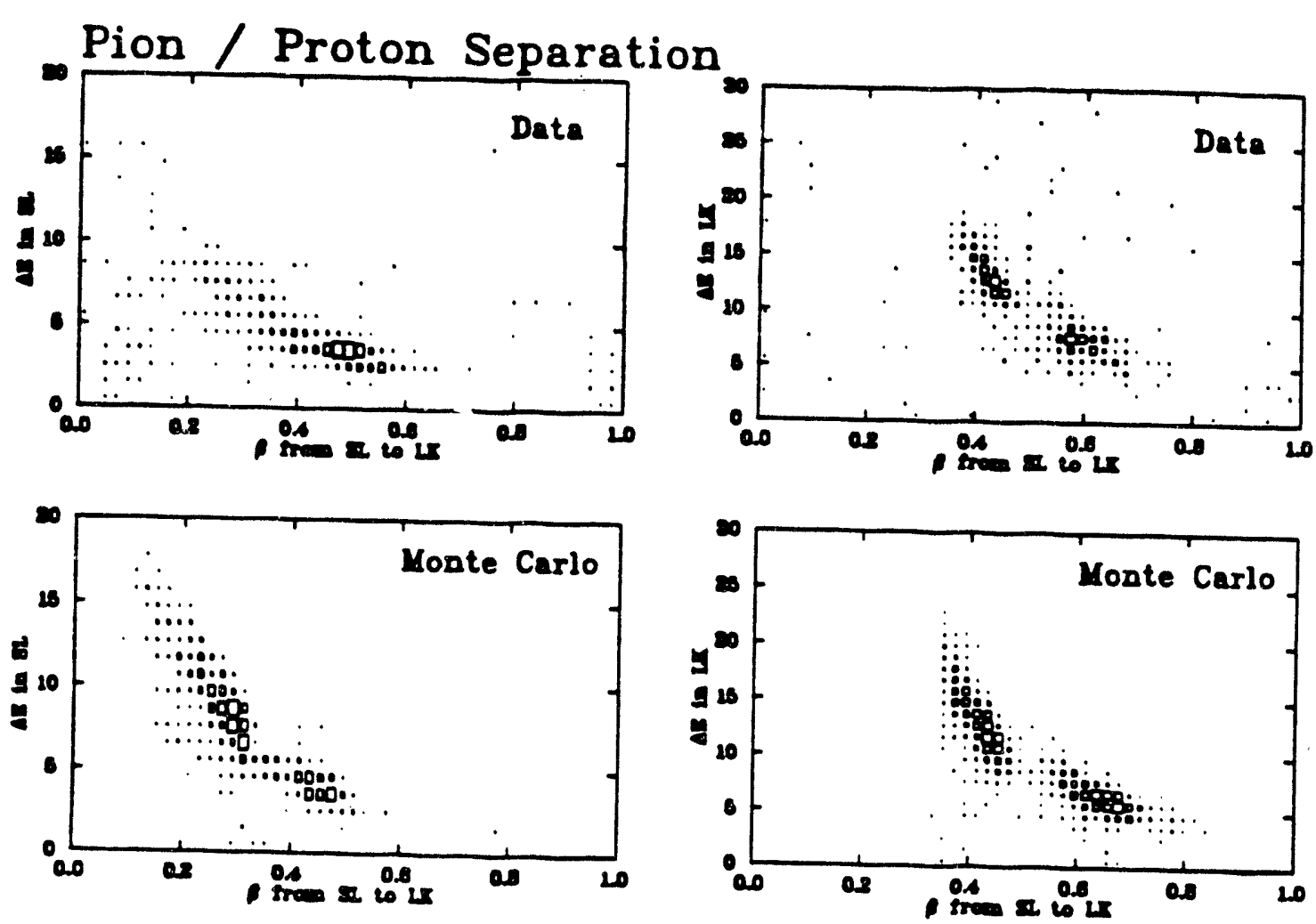

Figure 3.12 The proton / pion separation is compared to Monte Carlo in these $2-d$ histograms of energy deposition verses velocity fraction $\beta$. The top row is data - the bottom is Monte Carlo. The left two are energy deposition in the SL layer for events which stop in the LK layer. The right two plots are energy deposition in the LK layer for events which stop in the LN 1 layer. The Monte Carlo plots are derived from flat kinetic energy distributions. Pions cluster at higher kinetic energies and deposit less energy than protons. 


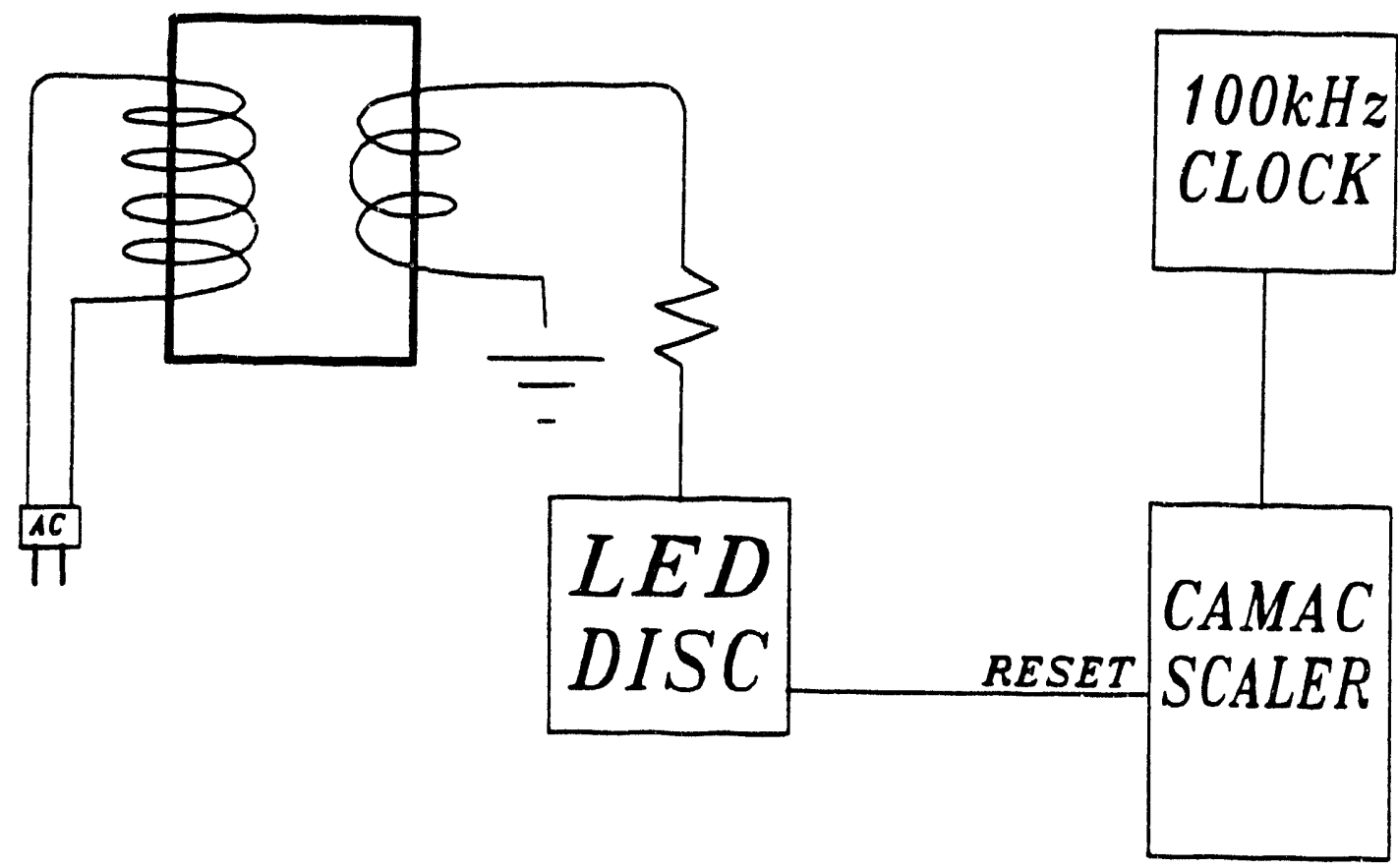

Figure 3.13 A 60 cycle signal is picked up from the local AC mains. The signal is discriminated and serves as the reset for a CAMAC scaler. A $100 \mathrm{kHz}$ free running clock is input to the scaler. Thus, the scaler value serves as the phase of the $60 \mathrm{~Hz}$ ambient background. 


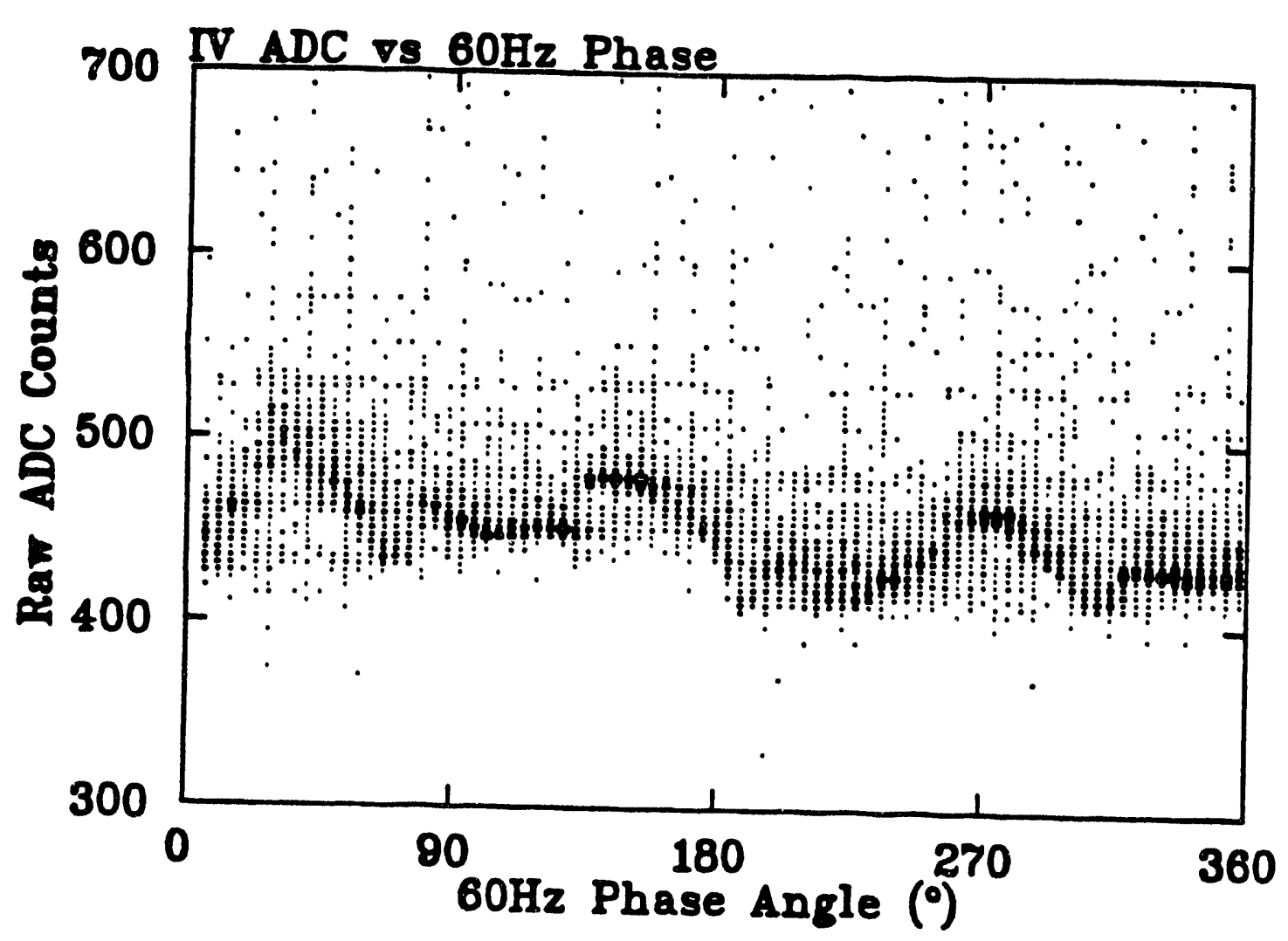

Figure 3.14 The ADC measurement of detector IV.0 is plotted versus the $60 \mathrm{~Hz}$ phase (arbitrary units). The effect of the time dependent ADC measurement effectively widens the pedestal and reduces the pedestal-signal
separation. 
ANALYSIS

○

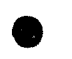

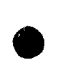

0

-

-

$=$

- 


\section{PHYSICS RESULTS}

The transformation of the raw numbers recorded by the experiment acquisition system into physical quantities is described in the previous chapter. In this chapter, I describe the evolution of these physical quantities into properties of the decay of the ${ }_{\Lambda}^{1} \mathrm{He}$ state. This includes (1) the hypernuclear lifetime, (2) the determination of the number of protons, pions, and neutrons originating from hypernuclear decay, (3) the total number of hypernuclei formed for normalization, and (5) the calculation of the various partial decay rates.

\subsection{Hypernuclear Lifetime Measurement}

The interval from the instant the $\left(K^{-}, \pi^{-}\right)$interaction takes place on a ${ }^{4} \mathrm{He}$ nucleus to the moment of the ${ }_{\Lambda}^{4} \mathrm{He}$ decay is the hypernuclear lifetime. The mean lifetime is expected to be of the order of the free $\Lambda$ decay mean lifetime[2], $\tau_{\Lambda}=263 \pm 2 p s$. The measurement of the mean lifetime of the hypernuclear system is accomplished through time and trajectories measurements of the incident $\mathrm{K}^{-}$and of the hypernuclear decay product. A schematic of the ${ }^{4} \mathrm{He}$ target area and how the lifetime measurement is made is given in figure 4.1.

The hypernuclear lifetime $t_{t}$ is the difference of the time of formation and the mean time of the decay. The formation time of the hypernuclear system is delermined from knowledge of:

1. The incident kaon's velocity and trajectory. The velocity of the incident kaon is determined from the momentum measurement from its trajectory through 


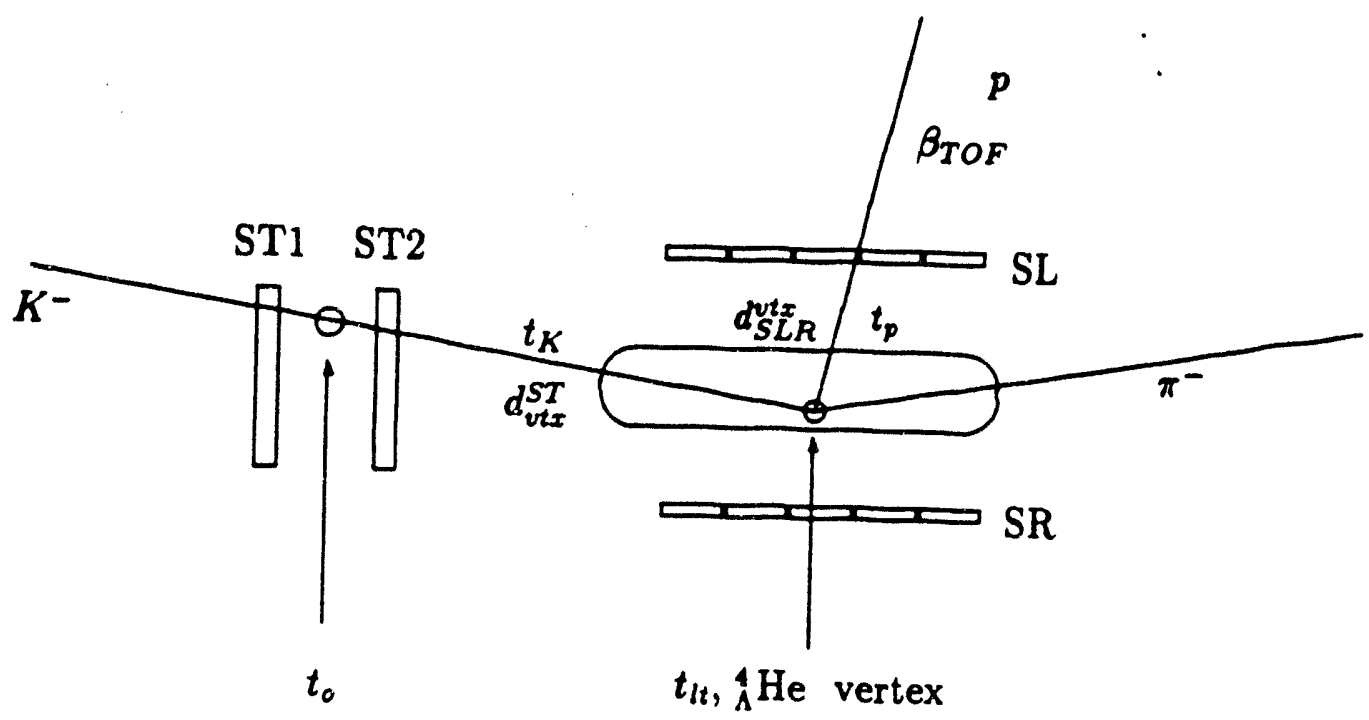

Figure 4.1 This schematic of the "He target area illustrates the lifetime measurement method. The mean time as a $K^{-}$passes the mean position of ST1 and ST2 is $t_{0}$. The time it takes the $K^{-}$to get from this point to the ${ }_{\Lambda}^{1}$ He vertex is $t_{K}$ as it travels a distance $d_{v t x}^{S T}$. The time $t_{p}$ it takes the decay proton to get from the vertex point to the $S L$ is determined from the distance $d_{S L R}^{u t x}$ and velocity obtained from corrections to $\beta_{t o f}$.

the upstream spectrometer. The velocity fraction is given by equation (3.6). The trajectory of the $K^{-}$is determined from the best fit to the hits in in-beam drift chambers.

2. A time measurement at a known position along the kaon's trajectory. The average of the mean times of the two ST's is the time $t_{0}$ that the $K^{-}$passes the midpoint between ST1 and ST2

$$
t_{0}=\frac{1}{2}\left(t_{\text {avg }}^{S T 1}+t_{\text {avg }}^{S T 2}\right) \text {. }
$$

This gives us a time and position along the $K^{-}$trajectory.

3. The vertex position of the $\left(\mathrm{K}^{-}, \pi^{-}\right)$vertex. The vertex position is determined by the distance of closest approach of the out-of-beam trajectory of the decay product and the $K^{-}$trajectory. In principal, we could also obtain the vertex position form the distance of closest approach between the $K^{-}$and $\pi^{-}$trajectories. 
However, the error on the $z$ vertex position goes rou ro $_{t} \frac{\sigma_{d}}{\sin \theta_{S A}}$ which becomes quite large at scattering angles, $\theta_{S A}$, approaching $\theta^{\circ}\left(\sigma_{d c}\right.$ is the position resolution of the in-beam drift chambers). The $x$ and $y$ vertex positions as determined by in-beam and out-of-beam methods are in good agreement with each other. The $z$ vertex positions are difficult to compare because of the poor inbeam resolution in that direction. To insure the out-of-beam track is correlated to the hypernucleus, we restrict the distance of closest approach to be within $2 \mathrm{~cm}$ (see figure 4.8 for the proton distance-of-closest-approach distribution).

\section{Velocity Corrections}

The decay time $t_{p}$ is derived from the velocity of the decay product between the target and SL/R and the distance between the vertex and the hit position of the SL/R. The calculation is complicated by the velocity corrections which must be made as the the particle passes through the liquid ${ }^{4} \mathrm{He}$ target. In general, a charged particle loses energy at a higher rate as it loses its kinetic energy. The velocity corrections in the target were broken into three regions: (1) $v_{c}$, inside the ${ }^{4} \mathrm{He}$ vessel, (2) $v_{b}$, outside the ${ }^{4} \mathrm{He}$ vessel but before the PVC shell, and (3) $v_{a}$, from the PVC shell region to the SL/R. Figure 4.2 is a schematic cross section of the ${ }^{4} \mathrm{He}$ target illustrating these three regions. The order in which corrections are made start from the SL/R and work back to the vertex.

A particles total energy $E$ is related to its velocity fraction $\beta$ by

$$
E_{t o f}=\frac{m c^{2}}{\sqrt{1-\beta^{2}}}
$$

In this experiment, $\beta$ is measured by time of flight between the SL/R and the L/RK detectors. This velocity is less than the original velocity of the particle since it lost a portion of its kinetic energy by energy deposition in the target area materials. The total energy of the particle at the vertex is

$$
E_{\circ}=E_{t o f}+\Delta E_{S L / R}+\Delta E_{P V C}+\Delta E_{H e}
$$

where $\Delta E_{x}$ is the energy lost in the various materials as the particle leaves the target.

The total energy of the particle before it traverses SL/R is $E_{t o f}+\Delta E_{S L / R} . \Delta E_{S L / R}$ is directly measured by the SL/R detectors (the measured value is in very good 
Cross Sectional View of Liquid Helium Target

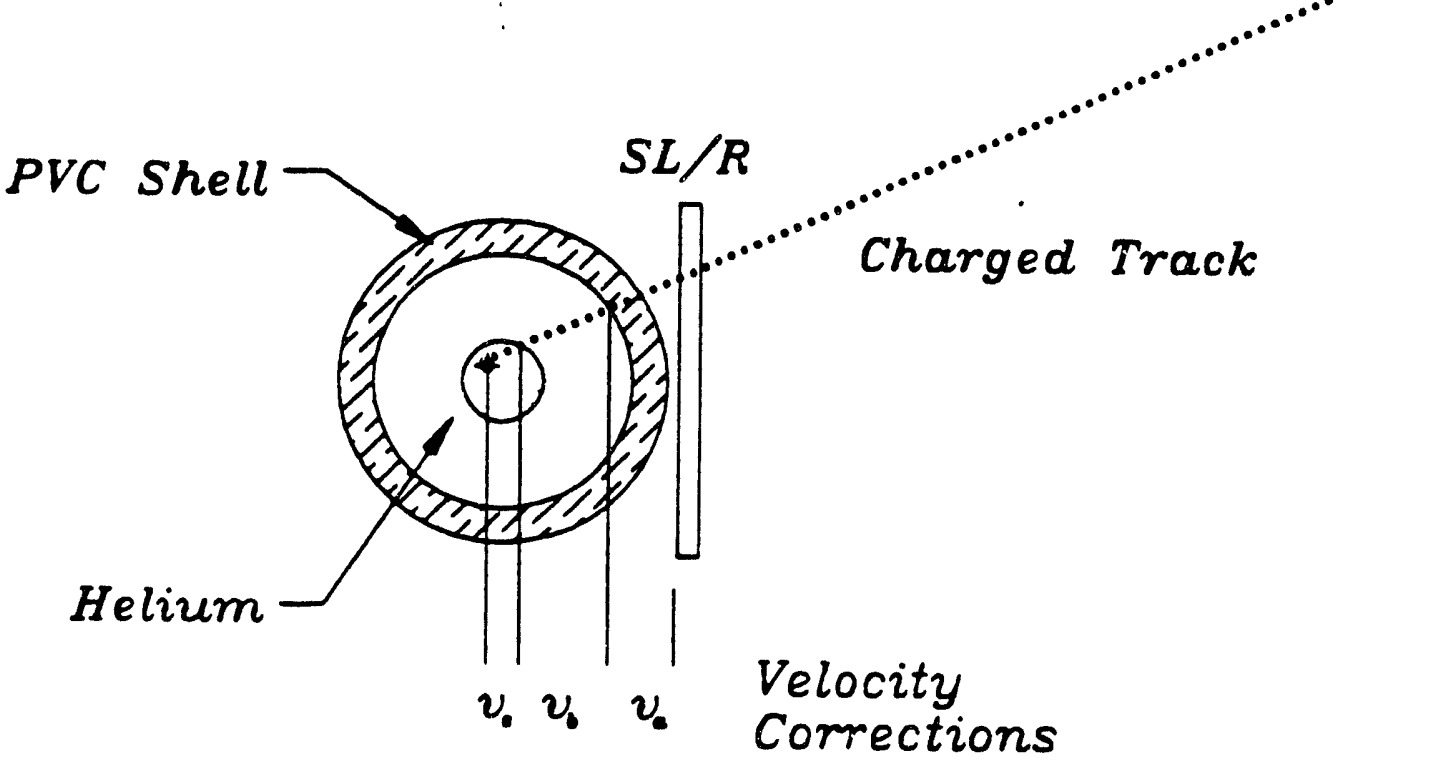

Figure 4.2 A schematic of a cross sectional view 4 He target area. As the charge particle exits the target, it loses kinetic energy from media interactions. Velocity corrections are made by averaging the exiting particle's velocity over the three regions to give $v_{a}, v_{b}$, and $v_{c}$, as indicated.

agreement with the calculated $\Delta E$ from $\frac{d E}{d x}$ ). Equation (4.2) is then inverted for $\beta$ to determine the velocity fraction before the SL/R interaction. This is then the velocity of the particle after it emerges from the ${ }^{4} \mathrm{He}$ vessel up to the SL/R counters.

For a given trajectory, the traversed distance through the PVC shell can be cal$=$ culated. The energy deposited in the shell $\Delta E_{P V C}$ is then inferred. Once we have the energy loos in the shell, we can calculate the velocity before the particle entered the shell. The velocity. from the inside of the shell and the SL/R are averaged to give the effective velocity between these points, $v_{a}$. The velocity $v_{b}$ is approximated to be a constant value of the velocity at the inside the shell.

The total energy lost in the ${ }^{4} \mathrm{He}, \Delta E_{H e}$, is calculated from the total distance of 
the trajectory in the liquid ${ }^{4} \mathrm{He}$ vessel and the kinetic energy of the particle after it emerges. The calculated velocity of the particle in the Helium target, $v_{c}$, is an average of the velocity at the vertex and the velocity at the edge of the vessel.

Once we have the velocity in the three regions, it is straightforward to get the flight time from $\sum \frac{d}{v_{i}}$.

\section{Data Extraction}

It is not necessary to retain the entire charge coincidence data set to determine the hypernuclear lifetime. An unbiased subset of these data is chosen to select excellent examples of hypernuclear decay. A summary of the cuts made upon the data set are:

1.* The incident particle on the ${ }^{4} \mathrm{He}$ target must be a $K^{-}$. This is a standard cut for all $\left(K^{-}, \pi^{-}\right)$event samples obtained from a time of flight cut between S1 and ST2.

2. Only decay protons are considered. Pions are not used since there is a slight uncertainty whether the emitted pions are from hypernuclear decay or from the quasi-free $\Lambda$ decay. The signal of protons emitted from hypernuclear decay is extremely clear. Also, the time response of the detector to protons can be studied (section 4.1). Also, we only consider those events with only one track emitted from the target.

3. Decay protons with kinetic energies between 70 and $120 \mathrm{MeV}$ are selected. The kinetic energy is determined from time of flight between SL/R and L/RK and energy loss corrections in the target. The uncertainty of the energy loss corrections in the target increases for lower energy particles.

4.* A two-sigma cut on the proton excitation energy is made This insures the sample is pure hypernuclear decay.

5. We selected only those events for which the vertex was found to be inside of the liquid ${ }^{4} \mathrm{He}$ vessel. The vertex is determined from the closest approach of the straight line trajectories of the kaon and out-of-beam tracks. 


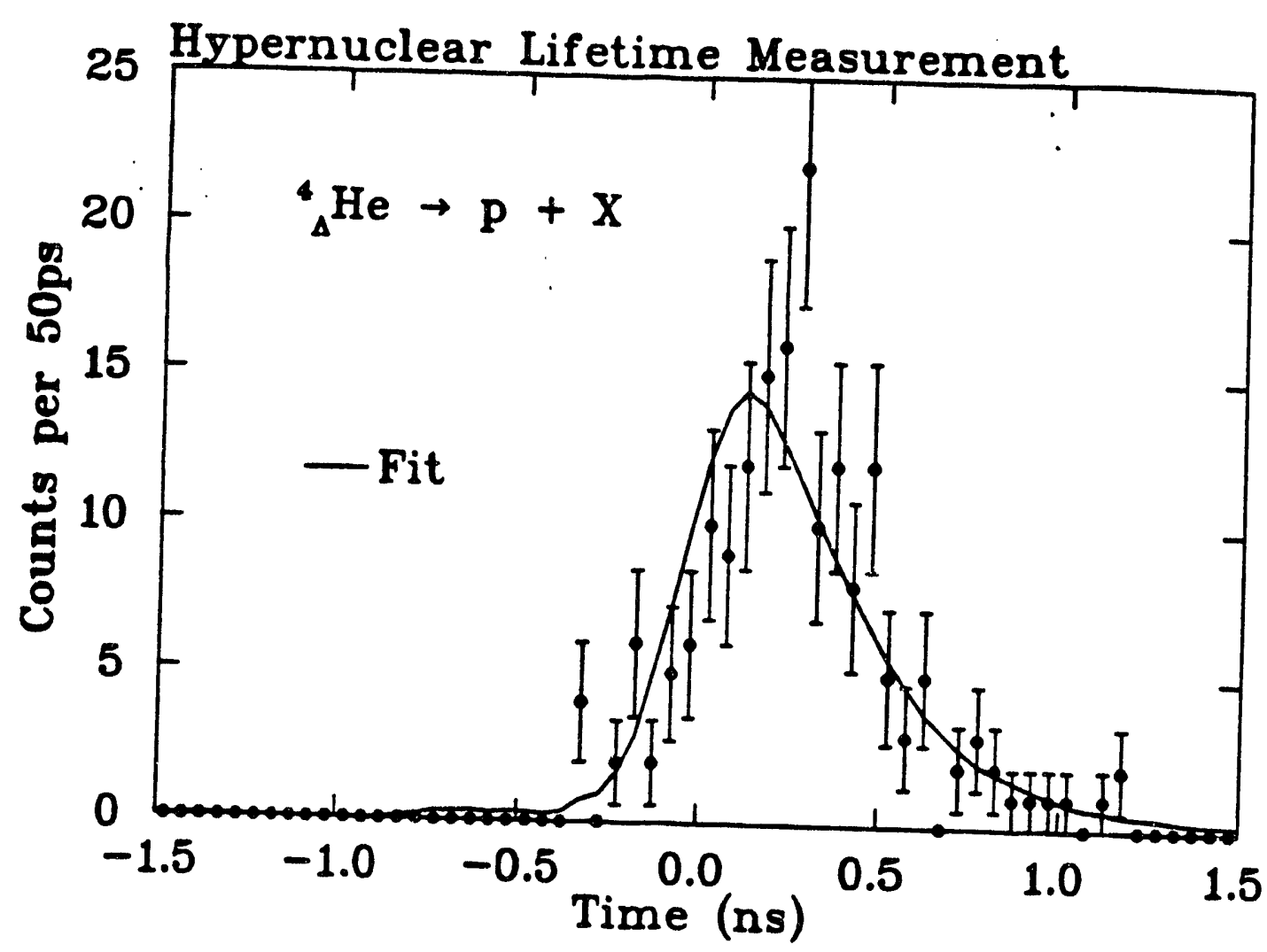

Figure 4.3 The lifetime distribution for hypernuclear events for a decay proton coincidence requirement. For other requirements, set text on page 81. The solid line histogram is the fit made to determine the lifetime.

6. The distance of closest approach between the out-of-beam trajectory and the $K^{-}$trajectory is required to be within $2 \mathrm{~cm}$. This is to insure a relation between the two tracks (see figure 4.8).

The resulting lifetime distribution for ${ }_{\Lambda}^{4}$ He decay is shown in figure 4.3.

\section{Lifetime Resolution}

The photon-statistics contribution to time resolution of a scintillator/PMT type counter is proportional to the square root of the amount of light produced in the scintillator. Protons are used for the lifetime measurement. It therefore makes sense to use prompt protons of the same energy range to study the timing response of the 
detector. One such source of protons comes from the reaction

$$
\pi^{-}+{ }^{4} \mathrm{He} \longrightarrow p+X
$$

A data sample was constructed with pions incident upon the ${ }^{4} \mathrm{He}$ target. The detected protons are then analyzed in exactly the same way as stimulated decay protons. The same cuts as described in the previous section (page 81) were applied with exception to those items marked with an '*'. The following items are substituted:

1. (refer to 1.* above) $\mathrm{A} \pi^{-}$is required to be incident upon the ${ }^{4} \mathrm{He}$ target as opposed to a $\mathrm{K}^{-}$.

2. (refer to 4.* above) The excitation energy cannot be calculated since there is no track requirement for the downstream spectrometer. Therefore, the excitation energy cut does not apply.

The resulting distribution is shown in figure 4.4. The deviation of this distribution from a Gaussian occurs primarily at the tails. Regardless, a Gaussian fit is made to the distribution and yields a resolution of $\sigma_{r f}=150 \mathrm{ps}$.

\section{Lifetime Fitting Procedure}

The rate per atom at which a particular nuclear unstable material disintegrates is a constant independent of all chemical conditions. Given a large quantity of the unstable material, the average number, $-d N$, that will disintegrate in a small time interval, $d t$, is proportional to the number of nuclei, $N$, present at time $t$; that is,

$$
-d N=\frac{1}{\tau} N d t
$$

where $\frac{1}{\tau}$ is the proportionality constant particular to that material. To get the probability distribution $P_{\tau}(t)$, this expression is integrated and normalized to the total number of nuclei,

$$
P_{\tau}(t)=\frac{1}{\tau} e^{-t / \tau}
$$

It is safely assumed that the time distribution for hypernuclear decay follows this relation. However, the distribution which is measured in the experiment will not behave as equation (4.6) since we are unable to measure time to infinite precision. The 


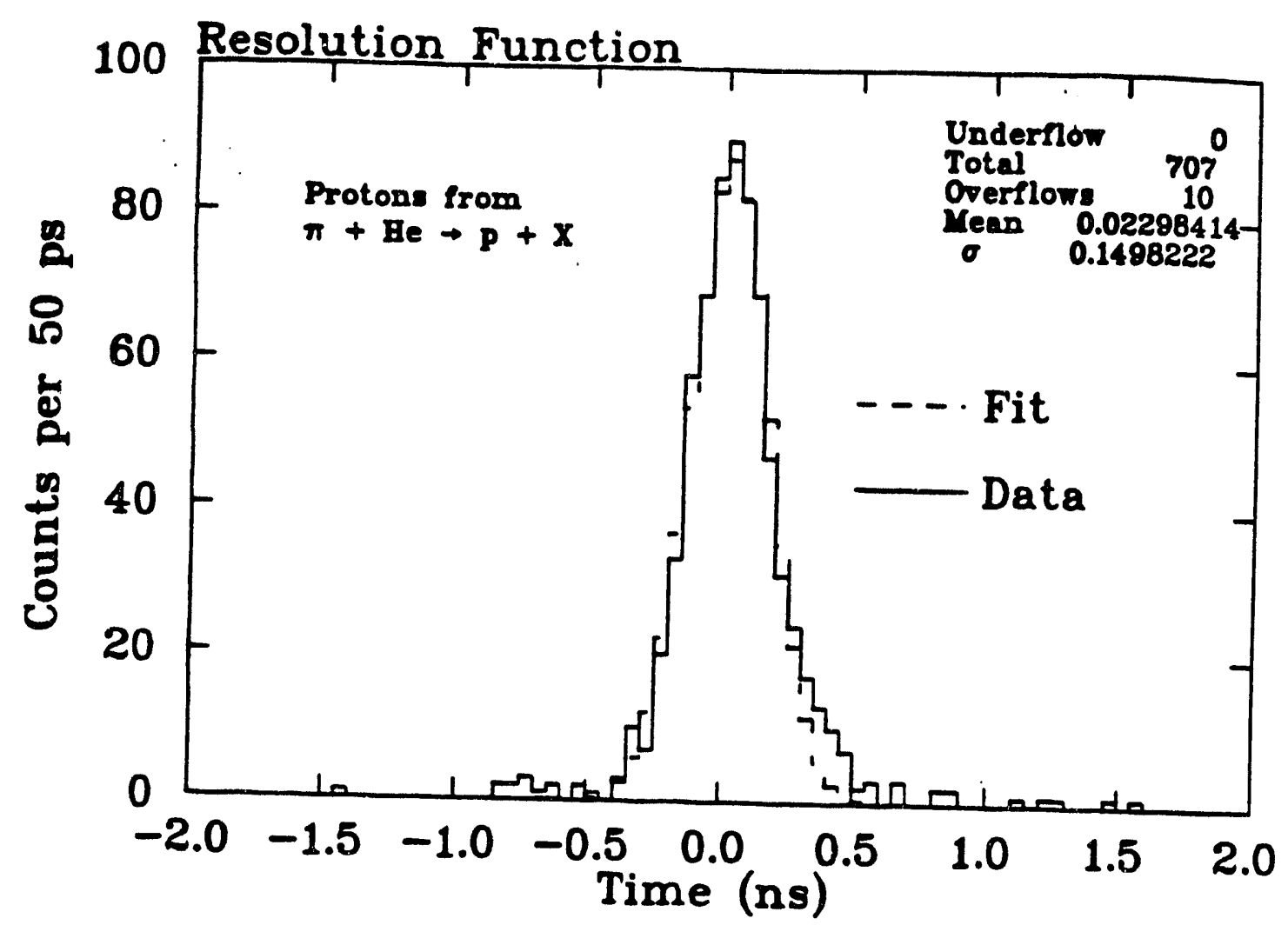

Figure 4.4 The 'lifetime' distribution for prompt protons from the reaction equation (4.4). The same requirements of decay protons in figure 4.3 are applied bere to the scattered protons with the exceptions given in the text. Also, overlayed is a Gaussian fit to the distribution. The distribution defines the resolution function for the lifetime measurement.

actual time distribution for our measurement $L_{\tau}(t)$ is expected to be a convolution of $P_{\tau}(t)$ with our time resolution function, $R(t)$,

$$
L_{\tau}(t)=\int_{-\infty}^{\infty} d t^{\prime} R\left(t^{\prime}\right) P_{\tau}\left(t-t^{\prime}\right)
$$

- The resolution function $R(t)$ is the measured time response of the detector to an infinity short 'lifetime' signal. It is a measure of $t \mathrm{t}: \mathrm{time}$ dispersion as a function of time of the precision tairget timing counters.

The form of $\dot{L}_{\tau}(t)$ is then dependent upon the choice of $R(t)$. The best ${ }^{1}$ choice is to let the data accumulated for the resolution measurement (figure 4.4) represent

\footnotetext{
${ }^{2}$ An alternative choice would be to pick a Gaussian function for $R(t)$ so that $L_{r}(t)$ could be solved
} 
$R(t)$. Thus, the integral in equation (4.7) becomes a sum of integrals over each time bin $t_{i}$,

$$
L_{\tau}\left(t_{j}\right)=\sum_{i=0}^{n} \frac{1}{t_{i+1}-t_{i}} \int_{t_{i}}^{t_{i+1}} d t^{\prime} R\left(t_{i}\right) P_{\tau}\left(t_{j}-t^{\prime}\right) .
$$

To account for a possible kackground of accidentals, the four parameter fit (including $\tau)$ made to the hypernuclear decay distribution is

$$
f\left(t_{j}\right)=C_{1}^{2}+C_{2} t_{j}+C_{3}^{2} L_{\tau}\left(t_{j}-t_{\circ}\right)
$$

where the constant $t_{0}$ is the mean position of $R(t)$ determined from a Gaussian fit.

The general criterion used in most fitting algorithms is to determine the best choice of parameters through the minimization of $\chi^{2} \cdot \chi^{2}$ is defined by

$$
\chi^{2}=\sum_{i=0}^{n}\left(\frac{y_{i}-f\left(x_{i}\right)}{\sigma_{i}}\right)^{2} .
$$

An assumption used in the derivation of $\chi^{2}$ is that the bin to bin errors are distributed according to Gaussian statistics. In the case where the number of counts per bin is high, a Gaussian approximation is valid. However, for a lifetime distribution, where the number of counts per bin is not necessarily large, especially in the tail of the distribution, this criterion does not necessarily apply.

In this analysis, we choose our set of parameters $\left\{\tau, C_{1}, C_{2}, C_{3}\right\}$ such that we obtain a minimum of $\Xi$, defined as,

$$
\Xi=\sum_{i=0}^{n} f\left(x_{i}\right)-y_{i}-y_{i} \ln \frac{f\left(x_{i}\right)}{y_{i}} .
$$

This criterion is derived from the rnaximization of the likelihood function. The likelihood function is the product of the probabilities that the fit equation represents the data for each bin

$$
M=\prod_{i=0}^{n} \mathcal{P}_{i}
$$

where the product is over bin to bin probabilities, $\mathcal{P}_{i}$. The $\chi^{2}$ criteria can be derived from equation (4.12) by choosing the Gaussian distribution for $\mathcal{P}_{i}$ and maximizing $M$.

analytically. However, the shortcoming of the approach is that a Gaussian representation of $R(t)$ is questionable because of the tails in the distribution (figure 4.4). 


\begin{tabular}{|c|c|}
\hline \multicolumn{2}{|c|}{$\Xi$ Minimization Results } \\
\hline \hline Parameter & Value \\
\hline$\tau$ & $245_{-23}^{+24} p s$ \\
$C_{1}^{2}$ & $0.00025 \pm 0.0012$ \\
$C_{2}$ & $0.0007 \pm 0.0012 \mathrm{ps}^{-1}$ \\
$C_{3}^{2}$ & $13.34 \pm 0.50$ \\
\hline
\end{tabular}

Table 4.1 The resultant fitted parameters to they hypernuclear lifetime distribution(figure 4.3) using equation 4.9.

The $\Xi$ derivation is carried through by choosing the Poisson probability distribution for $\mathcal{P}_{i}$. Equation (4.11) results from the maximization of $M$. It can also be shown[7] that a minimized $\Xi$ fit conserves the number of counts (unlike a $\chi^{2}$ minimization which systematically underfits the data). The results of the $\Xi$ minimization fit to the lifetime distribution (figure 4.3) to $L_{\tau}(t)$ (equation 4.7) are shown in table 4.1.

The error estimate is obtained[21] from a contour of $\Delta \Xi=1$ in the free parameter space. This contour corresponds to a 1 sigma deviation in the parameters. Since the coefficients of the constant and linear term in $\mathcal{P}_{i}$ are negligibly small, only a contour in the two dimensional parameter space of $\tau$ and $C_{3}$ is considered. The error estimates are also listed in table 4.1.

\subsection{Decay Product Measurements}

The reciprocal of the hypernuclear lifetime is equal to the sum of the individual partial rates,

$$
\frac{1}{\tau}=\Gamma_{t o t}=\Gamma_{p}+\Gamma_{n}+\Gamma_{\pi 0}+\Gamma_{\pi-}
$$
To determine the individual partial rates it is necessary to calculate the fraction of
the total hyr ernuclear decay for each mode,

$$
\Gamma_{i}=\frac{N_{i}}{N_{t o t}} \Gamma_{t o t}
$$

where $N_{i}$ is the total number of decays to the $i^{\text {th }}$ mode which occurred, and $N_{\text {tot }}$ is the total number of hypernuclei created. 


\subsubsection{Total Hypernuclear Rate}

During the experiment, the estimated number of $\left(K^{-}, \pi^{-}\right)$triggers per beam spill was about 120 . Most of these $\left(K^{-}, \pi^{-}\right)$triggers are not necessarily from hypernuclear formation but from background events such as kaon decays and random accidentals. Due to the limits of our acquisition system we were forced to be selective about which events to write to tape.

One of the triggers that was included in our acquisition hardware was a prescaled unbiased $\left(K^{-}, \pi^{-}\right)$trigger. This was unbiased in the sense that there wasn't any kind of requirement whatsoever in the out-of-beam detectors. The total number of hypernuclei produced in the course of this experiment is obtained by extracting the hypernuclear formation signal from this trigger.

The exact same cuts and criteria were used in the in-beam analysis of the unbiased $\left(K^{-}, \pi^{-}\right)$sample as were used in the other coincident $\left(K^{-}, \pi^{-}\right)$samples. That is, events with poor in-beam tracking or events which were consistent with kaon decay were removed (see section 3.1). The excitation energy for the total accumulated unbiased $\left(K^{-}, \pi^{-}\right)$sample is calculated and shown in figure 4.5. In this figure, the hypernuclear signal is quite evident at $\mathrm{EE}=0 \mathrm{MeV}$.

The extraction of the hypernuclear formation signal from this distribution is dependent upon our knowledge of the shape of the background. Our estimate of the shape is obtained from data accumulated while the ${ }^{4} \mathrm{He}$ vessel was empty. The spectrum for this sample is shown in figure 4.6. This data set was taken at the tail end of the AGS schedule for the ${ }^{4} \mathrm{He}$ data run. As a result, the statistics are limited.

The $\left(K^{-}, \pi^{-}\right)$background shape is smoothed by an algorithm developed by Savitzky and Golay[22]. The method conserves the area under the curve and yet preserves the zeroth, second, and higher moments. The comparison of the smoothed data to empty target data are also shown in figure 4.6.

At this point we assume that this smoothed spectrum is the shape of the background $\left(K^{-}, \pi^{-}\right)$spectrum. Since this choice of shape is based upon the actual data, it is probably a reasonable assumption.

The smoothed background is normalized to the excitation energy region between -40 and $-5 \mathrm{MeV}$. 


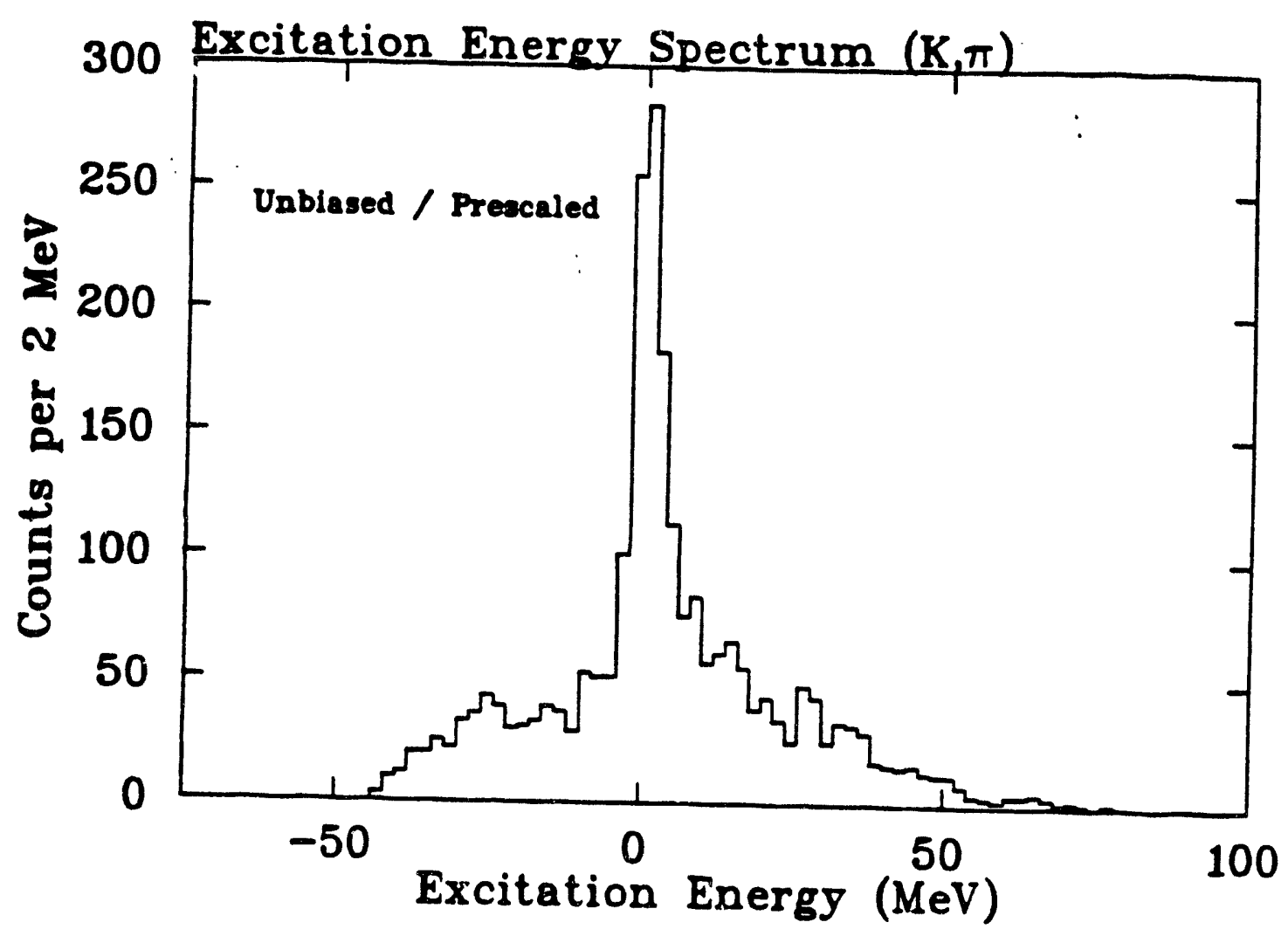

Figure 4.5 The excitation energy spectrum for prescaled unbiased $\left(K^{-}, \pi^{-}\right)$triggers. Kaon decays and events which failed tracking quality cuts are removed (exactly the same cuts for proton and pion coincideace samples). The hypérnuclear formation signal is easily seen at $0 \mathrm{MeV}$.

A fit to the $\left(\mathrm{K}^{-}, \pi^{-}\right)$unbiased data set is of the form

$$
E E_{u}=E E_{\text {empty }}+\frac{C}{\sigma \sqrt{2 \pi}} e^{-\frac{1}{2}\left(\frac{s-\mu}{\varphi}\right)^{2}}
$$

where $\mu$ and $\sigma$ are determined from a fit to the proton excitation energy spectrum $=$ (section 4.9), and $C$ is the floating parameter. A comparison of the fit to the data are shown in figure 4.7. Note the fit does not take into account the shoulder on the right of the peak; this is intentional. The events above the background at the shoulder are assumed to be pion tags in the out-of-beam detector array originating from the decay of quasi-free produced $\Lambda$ 's. These events are not related directly to hypernuclear decay. The integration of the Gaussian part of equation (4.15) gives the 


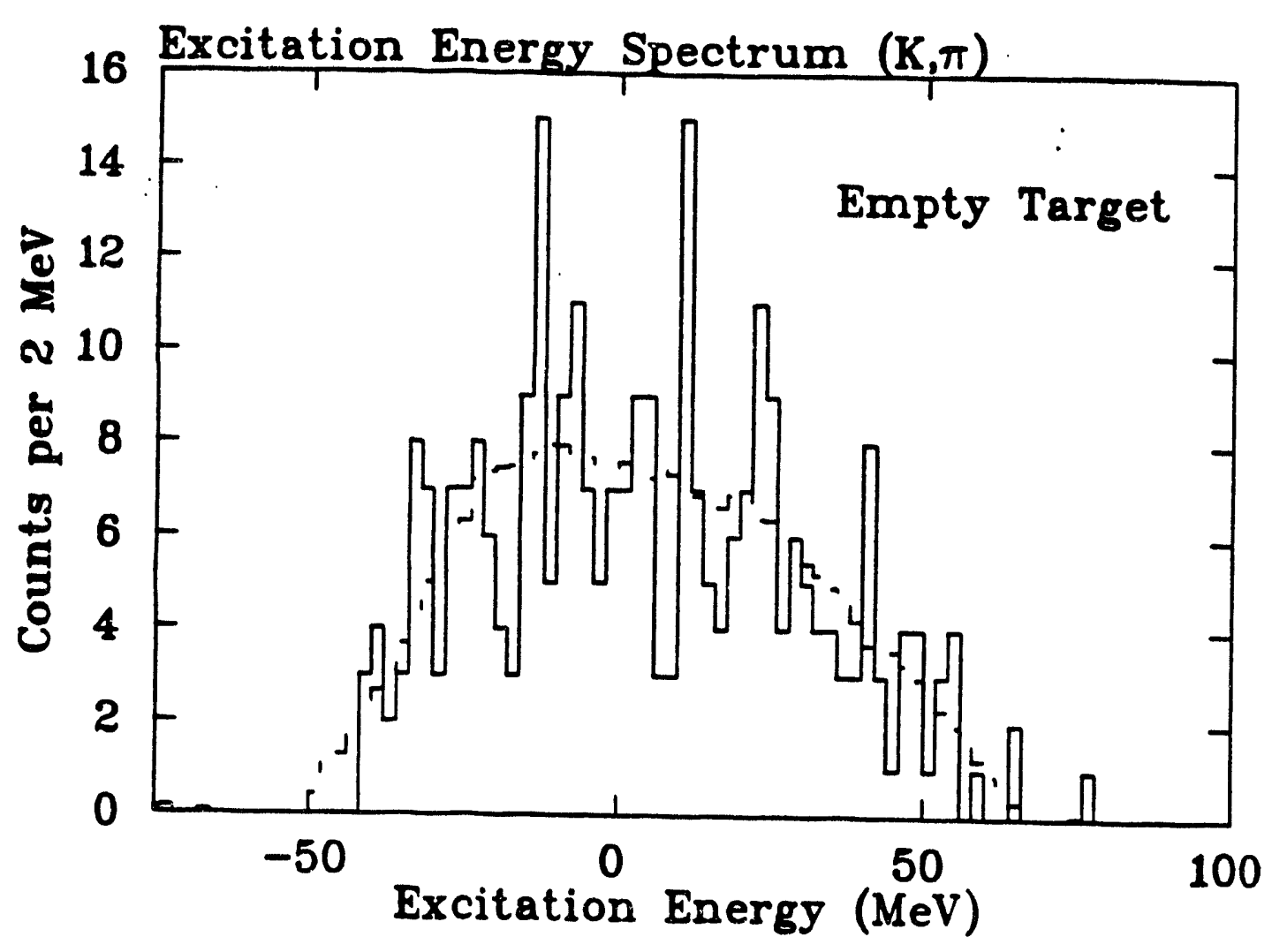

Figure 4.6 The excitation energy spectrum determined from empty ${ }^{4} \mathrm{He}$ target. Kaon decays and events which failed tracking quality cuts are removed (exactly the same cuts for proton and pion cinncidence samples). The data are smoothed using a 4th order Savitsky-Golay filter and plotted with a dotted line.

total number of $\left(K^{-}, \pi^{-}\right)$events in the sample. The result is

$$
N_{K \pi}=626 \pm 46 \text {. }
$$

To obtain the total number of $\left(\mathrm{K}^{-}, \pi^{-}\right)$events produced in the course of this experiment, $N_{K \pi}$ is multiplied by the hardware prescale factor used through the data accumulation,

$$
N_{K \pi}^{\text {rot }}=N_{K \pi} \times 20=12520 \pm 922 .
$$

The error is partially determined by varying $\sigma$ and $\mu$ of equation (4.15) in accordance to the errors found in determining the fit to the proton excitation spectrum. These errors are added in quadrature to give the error in $N_{K \pi}$ to give a net error on $N_{K \pi}^{\text {tot }}$. 


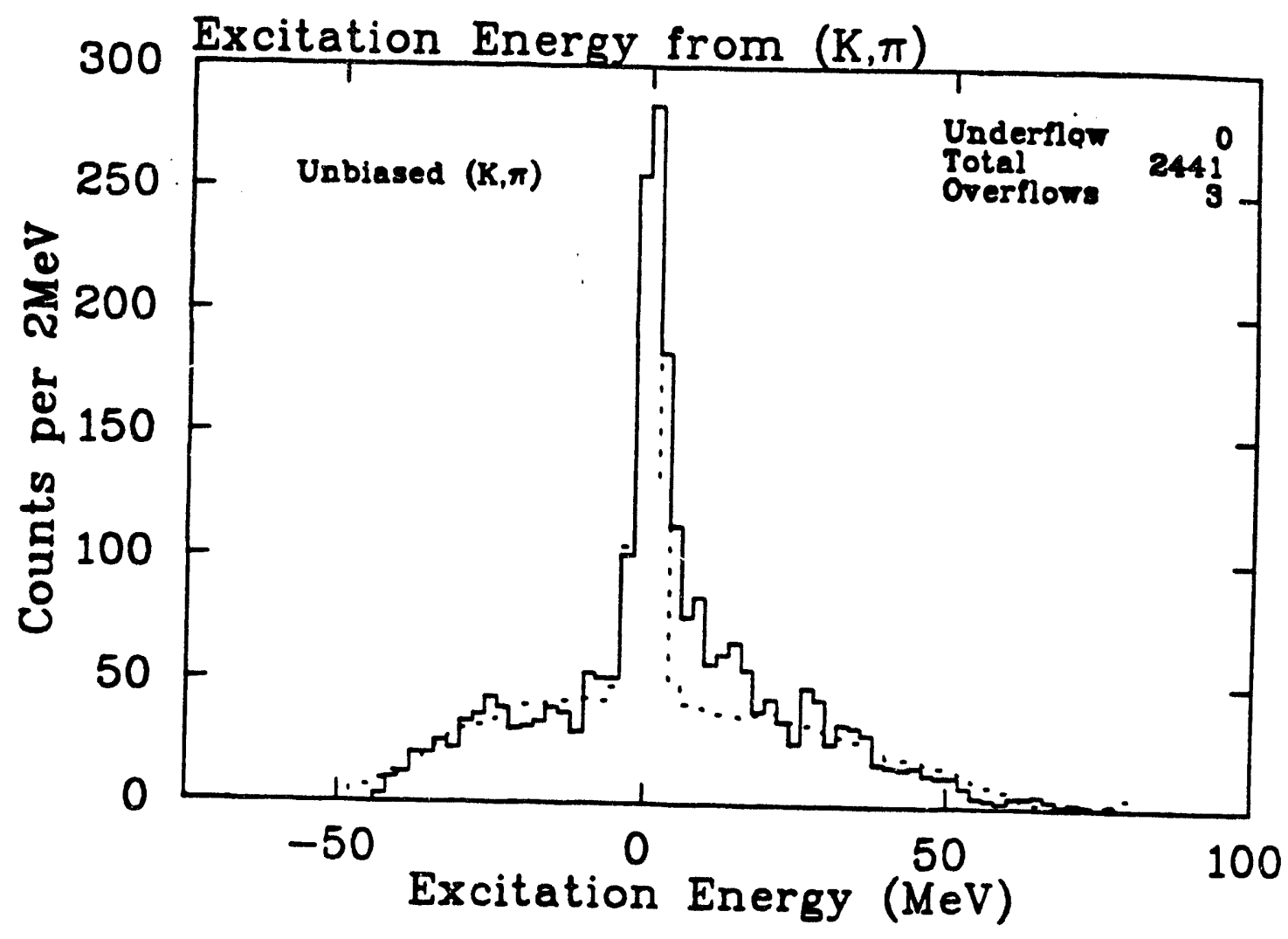

Figure 4.7 The solid line is the fit to the data from equation (4.15). The dashed line is the unbiased prescaled $\left(K^{-}, \pi^{-}\right)$data set. The shoulder on the right of the peak is assumed to be from quasi-free $\Lambda$ production.

\subsubsection{Proton Measurement}

The proton partial rate is the fraction of the total number of hypernuclear events which decayed via proton stimulated decay. A description of the methods used for proion identification and the $\left(K^{-}, \pi^{-}\right)$sample selection is found in sections 3.4 .2 and 3.1.

The measurement of the proton stimulated decay rate involves more than simply counting the number protons observed because:

- The protons from stimulated decay, $p+\Lambda \longrightarrow p+n$, are not the only source of protons observed in the detector. Other sources include 
1. Final state interactions from proton stimulated decay. The $p$ and/or $n$ which are liberated in the $p \Lambda$ interaction can scatter off of the residual nucleons.

2. Final state interactions from neutron stimulated decay. Either of the neutrons liberated in the $n \Lambda$ interaction can scatter off of the residual two protons.

3. Pions produced from hypernuclear mesonic decay interact with the target to produce protons. Pion absorption has been studied[23]. In perspective of the low probability of proton emission from pion absorption in the small amount of ${ }^{4} \mathrm{He}$ available, the likelihood of this event is negligible.

- The number of protons measured must be adjusted to represent the total number of protons produced. The detector is sensitive only to a limited fraction of $4 \pi$ steradians (approximately $\frac{1}{3}$ ). The limited efficiency is also dependent upon the type and energy of the particle. A careful energy dependent efficiency is investigated through detector modeling and simulation (see section 3.5).

A detailed discussion of the proton measurement is taken up in the following sections.

\section{Extracting Proton Sample}

An event sample is constructed with the following requirements:

- High quality in-beam tracks are selected where (a) a kaon is identified incident upon the ${ }^{4} \mathrm{He}$ target, (b) kaon decays are removed, and (c) events with poor tracking residuals are eliminated.

- Each event is required to have one or two detected protons, zero to two detected neutrons, and zero detected pions.

- A cut is placed upon the measured lifetime at $-0.75 \mathrm{~ns}$ to $1.75 \mathrm{~ns}$ (this corresponds to about $-3 \tau$ to $+7 \tau$ where $\tau$ is the hypernuclear life time). This is done to reduce any tracks in the out-of-beam detectors which are not correlated to the $\left(K^{-}, \pi^{-}\right)$vertex. 


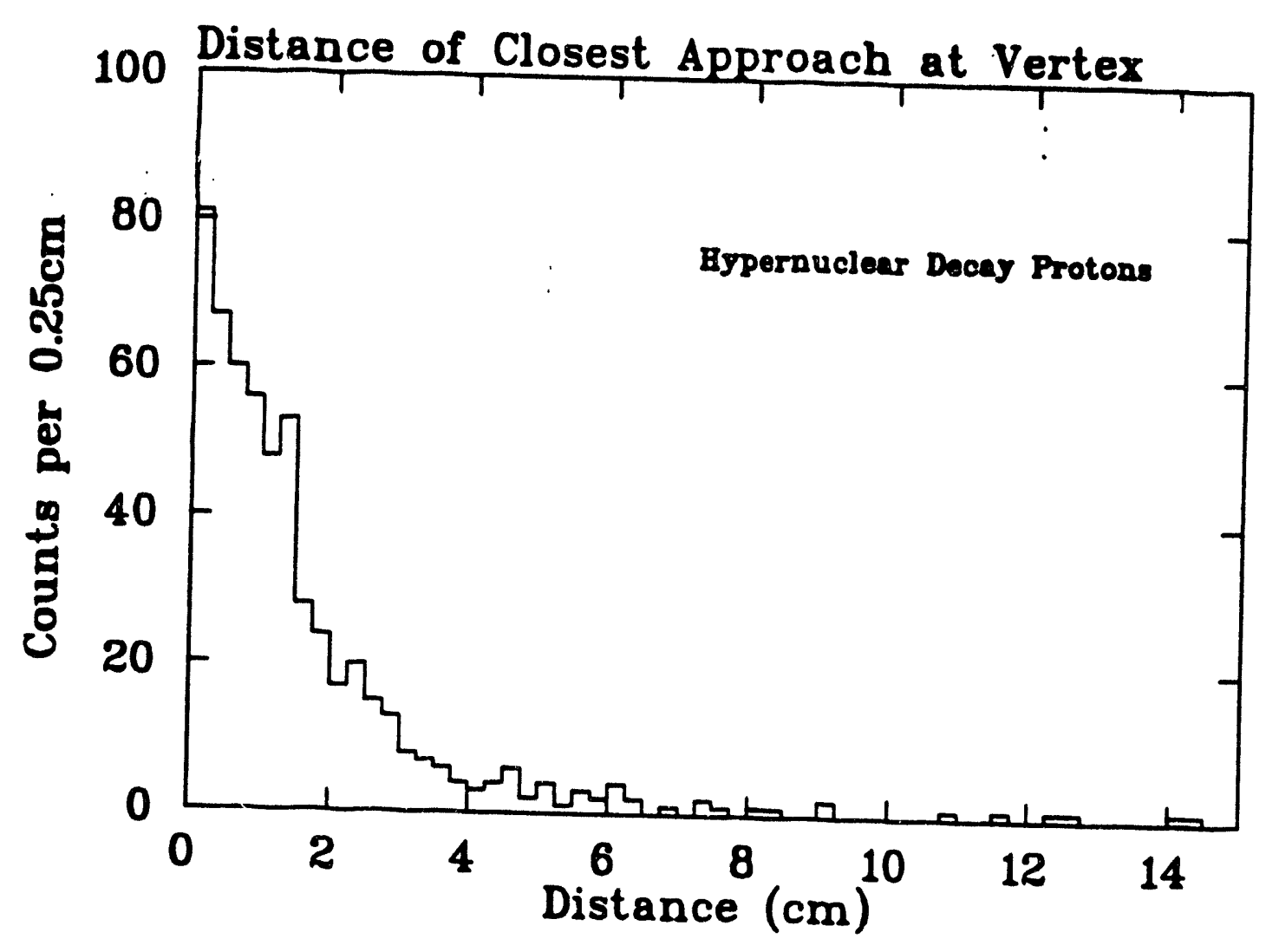

Figure 4.8 The distance of closest approach between the in-beam kaon trajectory and the out-of-beam identified proton is skown. A $2 \sigma_{E E}$ cut is placed upon the identified proton excitation energy of thase events.

- A cut on the distance of closest approach between the kaon trajectory and the out-of-beam traject-ry is set at $3 \mathrm{~cm}$. The distribution for the distance of closest approach before this cut is presented in figure 4.8.

The excitation energy spectrum for the coincident proton sample is shown in figure 4.9. This surprisingly clean spectrum is almost void of events outside or the region - of the signal peak at $\mathrm{OMeV}$.

The spectrometer energy resolution is determined from the proton spectrum. The rising edge of the peak is fit to a Gaussian distribution,

$$
E E=\frac{A}{\sigma \sqrt{2 \pi}} e^{-\frac{1}{2}\left(\frac{2-\mu}{\alpha}\right)^{2}}+C
$$




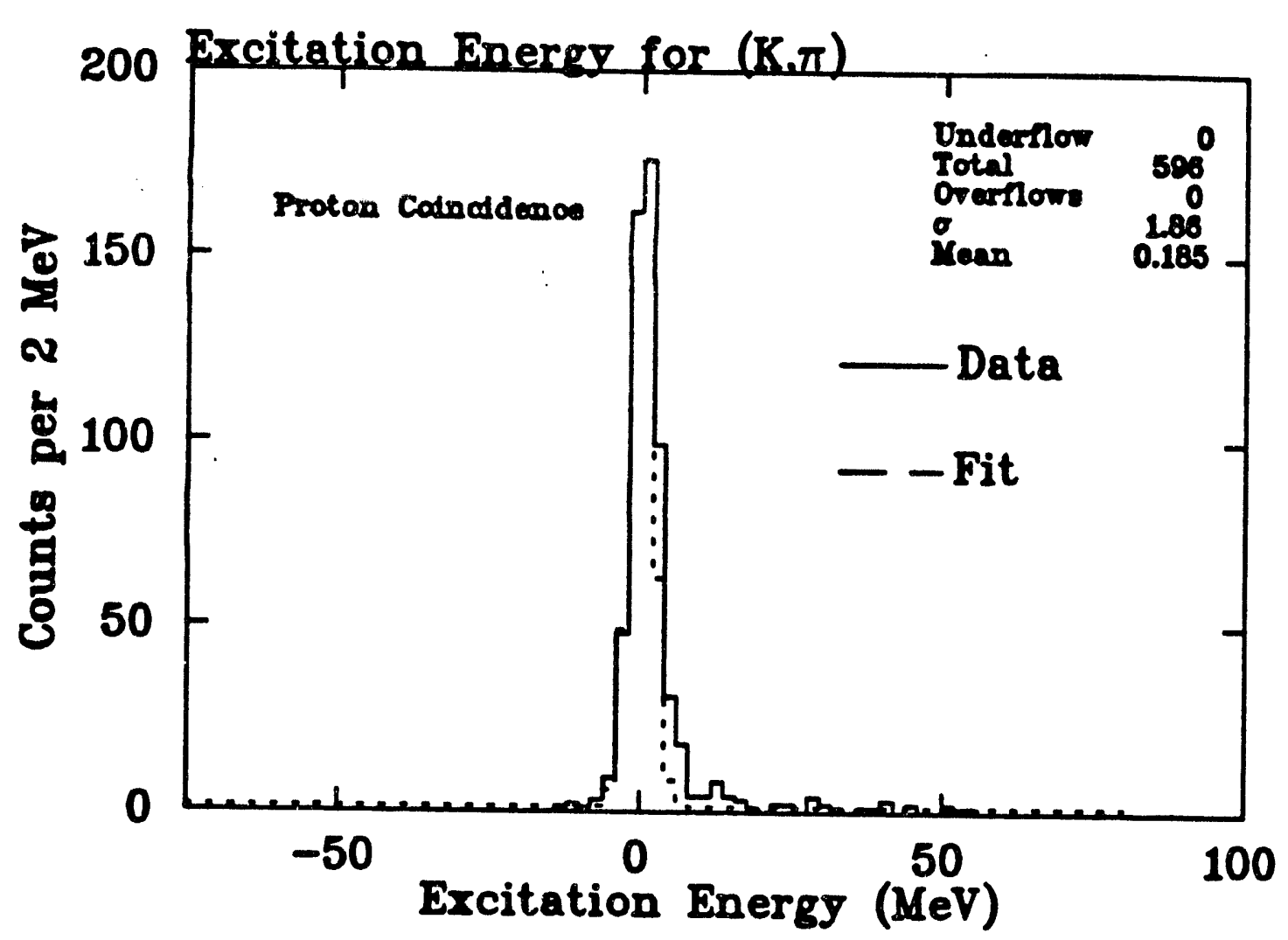

Figure 4.8 The excitation energy spectrum for identified protons. The minimum cuts included in the generation of this histogram include (1) requiring a $\left(K^{-}, \pi^{-}\right)$formation reaction, (2) removal of the background reaction $K^{-} \rightarrow \pi^{-}+\pi^{\circ}$, and (3) requirement of a proton in the out-of-beam detector array. A Gaussian fit is made to this distribution and is used as a measure of the spectrometer resolution.

with four free parameters; $A, \mu, C$, and $\sigma$. The result of the fit is overlayed in figure 4.9. The parameters of the proton fit are given in table 4.2.

The total number of raw observed protons is determined from integrating the $=$ Gaussian shape of the excitation energy spectrum, and is found to be

$$
N_{p}^{o b e}=456 \pm 24
$$

The error quoted is determined from refitting the spectrum with the worst case choice of parameters of table 4.2 within errors. 


\begin{tabular}{|c|c|}
\hline \multicolumn{2}{|c|}{ Proton EE Fit Parameters } \\
\hline \hline parameter & value \\
\hline A & $456 \pm 24$ \\
\hline$\mu$ & $0.185 \pm 0.040 \mathrm{MeV}$ \\
\hline$\sigma$ & $1.86 \pm 0.046 \mathrm{MeV}$ \\
\hline$C$ & $1.32 \pm 0.60$ \\
\hline
\end{tabular}

Table 4.2 Results of a fit to the proton coincident excitation energy of figure 4.9 using equation (4.18).

\section{Acceptance Correction}

A cut is placed upon the proton excitation energy spectrum to insure we have a sample of protons exclusively from hypernuclear decay. The cuts are placed to accept events between $\mu-2 \sigma$ to $\mu+2 \sigma$. The number of events in this sample is $N_{p}^{2 \sigma}=483$.

The initial kinetic energy $E_{\circ}$ for this sub-sample is calculated in a manner described by equation (4.3). The kinetic energy for the hypernuclear protons is shown in figure 4.10. To obtain the actual proton kinetic energy distribution it is necessary make acceptance corrections to the data of figure 4.10. An acceptance correction is performed upon the kinetic energy distribution. For a description of the correction procedure, see section 3.5 .

The acceptance corrected proton kinetic energy distribution, normalized to the number of hypernuclei produced, is shown in figure 4.11. The low end of the energy acceptance is at about $35 \mathrm{MeV}$. We essentially have no information on the shape of the distribution below this limit. The closest thing to a model independent result for proton spectrum from hypernuclear decay is given in figure 4.11 .

\section{Interpretations and Final Extraction}

To reiterate, the objective of the proton measurement is to obtain the proton stimulated decay rate. To do this, we need the total number of protons emitted in nucleon stimulated decay. Unfortunately, we have no direct way of measuring the low energy protons.

We rely upon an impulse approximation of a geometric model of the decaying 


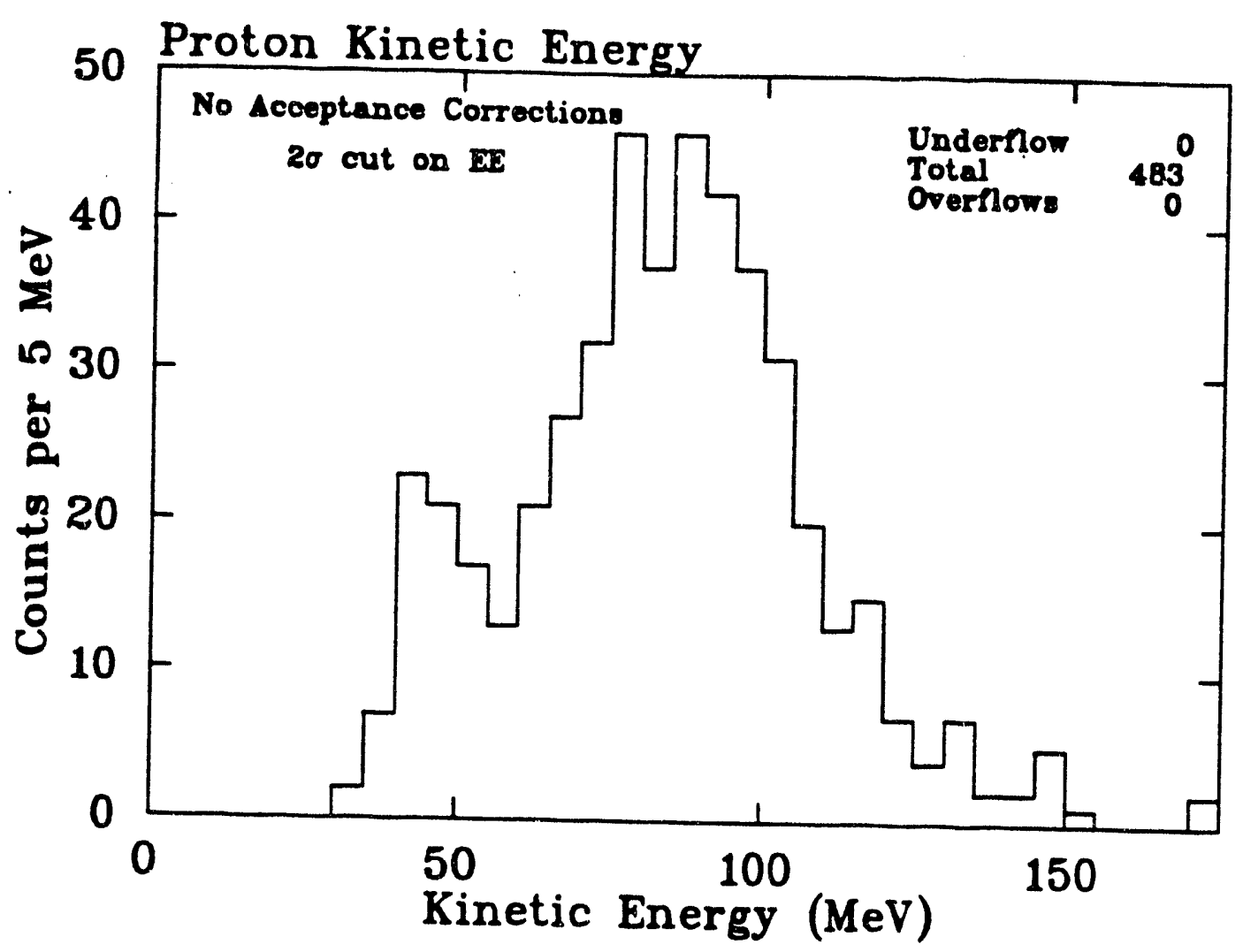

Figure 4.10 The initial kinetic energy of protons obtained from proton decay. A $2 \sigma_{E E}$ cut is placed upon the identified proton excitation energy of these events.

hypernucleus. The decay process is split into two separate problems: (1) nucleon emission independent of any final state interactions, and (2) the emitted nucleons interact with the residual nucleons. For brevity, we will refer to the first problem as no-scatter and the second case as one-scatter. The nodel is not intended to determine the absolute kinetic energy distributions - merely the shapes. We can then fit the shapes to the data to determine their relative importance.

In both cases, the nucleus is modeled as four s-wave particles with momentum derived from a Gaussian distribution of width $\frac{1}{\sqrt{3}}(1.6 \mathrm{fm})^{-1}$. This is based upon simple harmonic oscillator wave functions confined to the extent of the Helium dimensions. The factor of $\frac{1}{\sqrt{3}}$ is necessary to get the momentum for each direction.

For the no-scatter case, a momentum for a nucleon and the $\Lambda$ is derived from this distribution. The momentum of the residual nucleon pair is thus determined. Total 


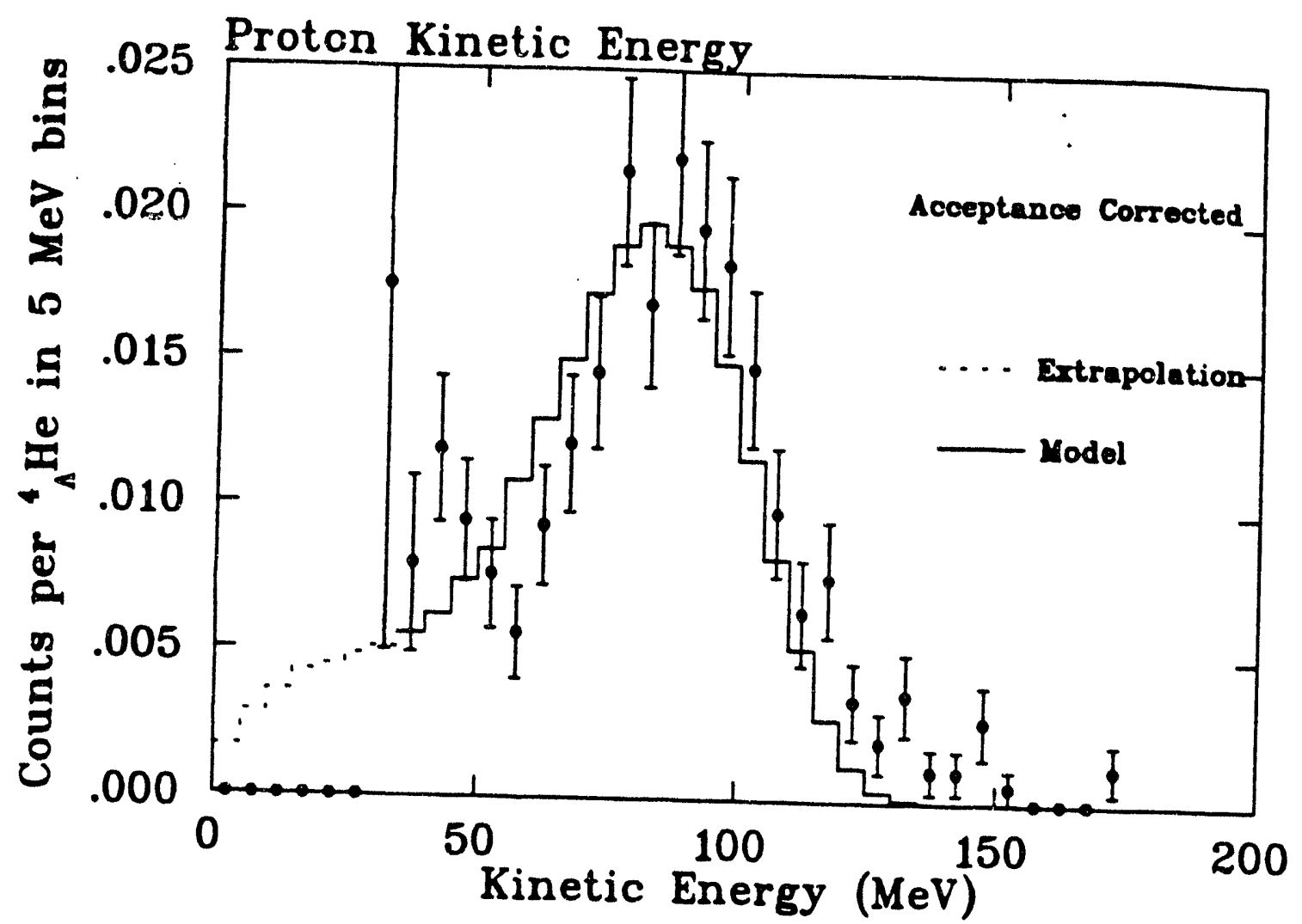

Figure 4.11 The acceptance corrected kinetic energy distribution for protons from hypernuclear decay. The distribution is normalized to the total number of hypernuclei produced. The proton partial rate as a function of energy is obtained by multiplying the $y$ coordinate by $\Gamma_{\text {tot }}$. The solid line is the best fit to the distribution for modeled nucleon emission.

energy is conserved at this point but the $N \Lambda$ pair are not confined to the mass shell. Subsequently, the $N \Lambda$ decays to $N N$. The kinetic energy of one of these nucleons is made into a histogram.

The one-scatter takes the above representation one step further. One of the energetic nucleons from the $N \Lambda$ interaction subsequently scatters off of one of the two - spectator nucleons. The incrementation of the kinetic energy histogram is weighted by a factor of $1 / T$, where $T$ is the kinetic energy of the energetic nucleons in the reference frame of the spectator nucleons. This last factor is derived from the energy dependence of cross section for nucleon-nucleon scattering. Figure 4.12 shows the shape of the two distributions for no-scatter and one-scatter.

A two parameter weighted fit is made from normalized versions of these distribu- 


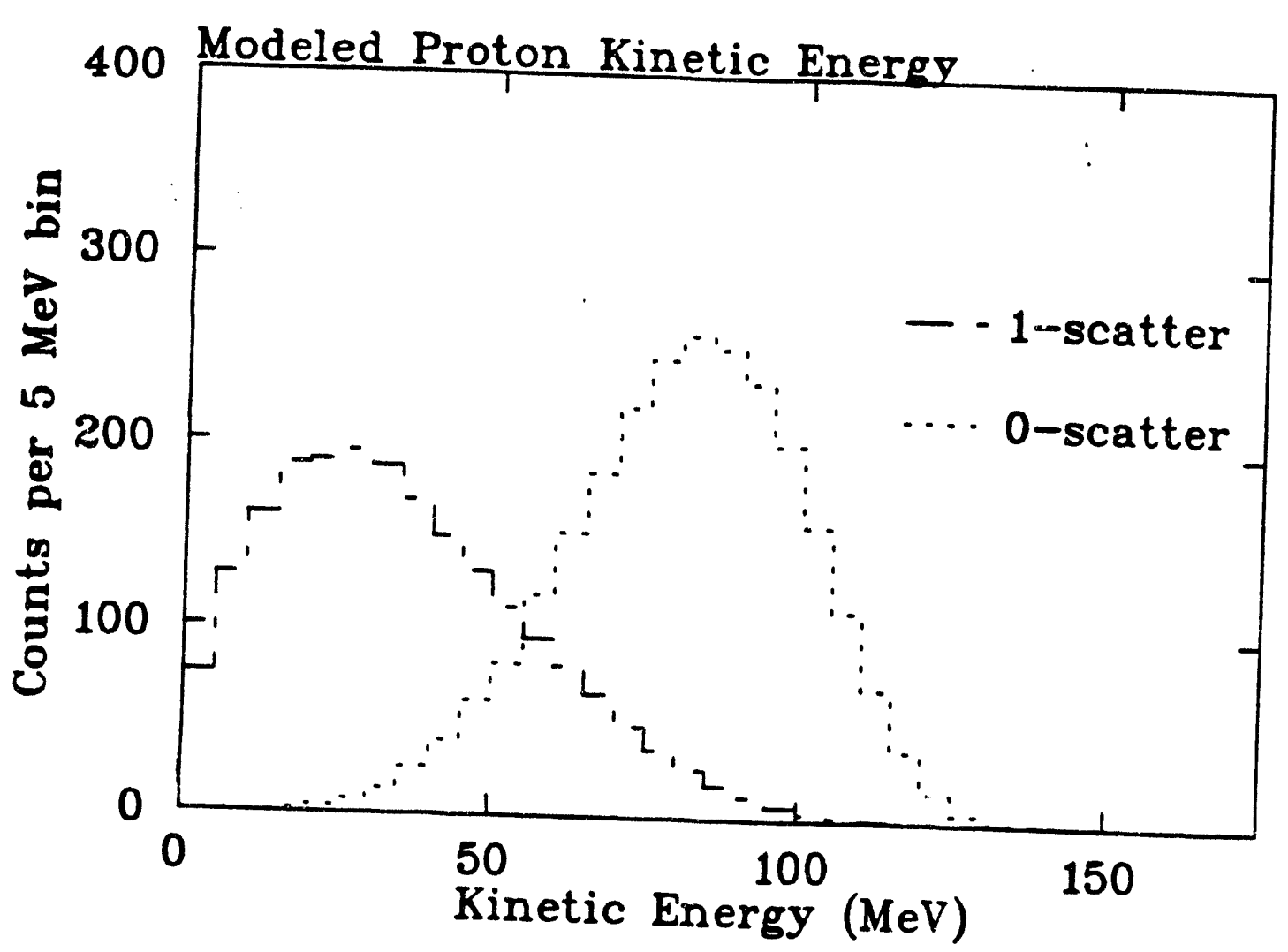

Figure 4.12 The kinetic energy distributions are modeled from simple nucleon emission (no-scatter) and emission which subsequently scatters off of the residual nucleus (one-scatter).

tions to the data of figure 4.11. The integrated number of counts for no-scatter and one-scatter fitted distributions are

$$
N_{0,}=2307 \pm 159 \quad N_{1}=576 \pm 256 .
$$

The combined total number of protons is

$$
\therefore \quad N_{p}^{c o r}=\frac{N_{p}^{e e}}{N_{2 \sigma}}\left(N_{0,}+N_{1 \triangleleft}\right)=2722 \pm 342 .
$$

The fit is compared to the data in figure 4.11. The extraction of the total proton stimulated decay rate is carried out later this chapter. 


\subsubsection{Pion Measurement}

To calculate the pion partial decay rate $\Gamma_{n}$ it is necessary to determine the fraction of the total number of hypernuclear events which subsequently decayed via pion emission. A detailed discussion for extracting $\left(\mathrm{K}^{-}, \pi^{-}\right)$events as well as pion identification was presented in sections 3.1 and 3.4.2. To summarize this reduction,

1. Quality in-beam tracks are selected where (a) a kaon is identified incident upon the ${ }^{4} \mathrm{He}$ target, (b) kaon decays are removed, and (c) events with poor tracking residuals are eliminated.

2. Pions are identified in the out-of-beam detector array. This is achieved by range, $\frac{d E}{d x}$ info: mation, and velocity measurements.

We also require events with only a single track - an identified pion. The excitation energy spectrum for identified single pions is shown in figure 4.13. Its distinguishing feature when compared to the proton spectrum is the extended shoulder on the right of the peak. A probable source for the shoulder is the decay of quasi-free $\Lambda$ 's. That is, a $\Lambda$ is produced from the ${ }^{4} \mathrm{He}\left(K^{-}, \pi^{-}\right)$reaction, but the $\Lambda$ has too much kinetic energy to be bound. Therefore, pions from hypernuclear decay can be isolated by fitting the left edge of the excitation energy peak to the spectrometer resolution distribution. A flat background parameter is also included in the fit in the same spirit of the proton excitation energy fit. The fit is illustrated in figure 4.13.

The integrated number of pions in this two parameter fit is

$$
N_{\pi}^{e e}=349 \pm 21 .
$$

The error quoted is determined from refitting the spectrum with the range of parameters of the proton excitation energy (table 4.2).

\section{Acceptance Correction}

Acceptance corrections are made in the same manner as for the proton measurement. The excitation energy cut, $\mu-2 \sigma$ to $\mu+2 \sigma$ are placed upon the pion excitation energy spectrum to insure that most of the identified pions originated from hypernuclear decay, and not quasi-free $\Lambda$ decay. The resulting number of events in this sub-sample 


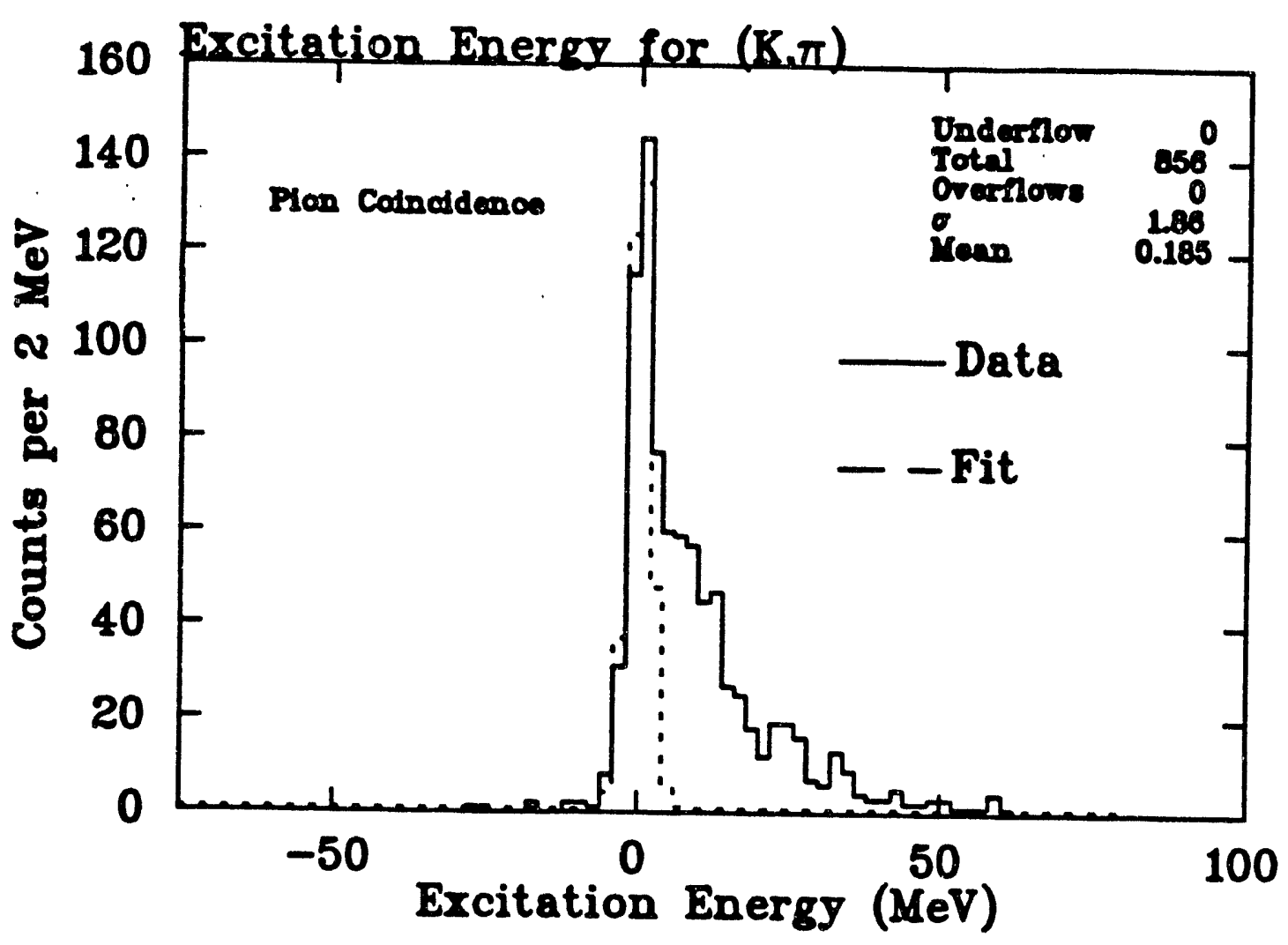

Figure 4.13 The excitation energy spectrum for identified pions. The minimum requirements included in the generation of this histogram include (1) requiring a $\left(\mathrm{K}^{-}, \mathrm{x}^{-}\right)$formation reaction, (2) removal of the background reaction $K^{-} \rightarrow \pi^{-}+\pi^{\circ}$, and (3) requirement of a pion in the out-of-beam detector array. The left edge of the peak is fitted to a Gaussian with width and mean characteristic of the spectrometer resolution.

is $N_{\pi}^{20}=362$. The kinetic energy distribution for these events before acceptance correction is shown in figure 4.14.

The acceptance correction is made in $5 \mathrm{MeV}$ bins - just as in the proton case. A description of the correction procedure is found in section 3.5. The resulting pion coincident kinetic energy distribution is shown in figure 4.15. This is the closest we can come to a model independent measurement of the $\pi^{-}$spectrum from mesonic ${ }_{\Lambda}^{4}$ He decay. 


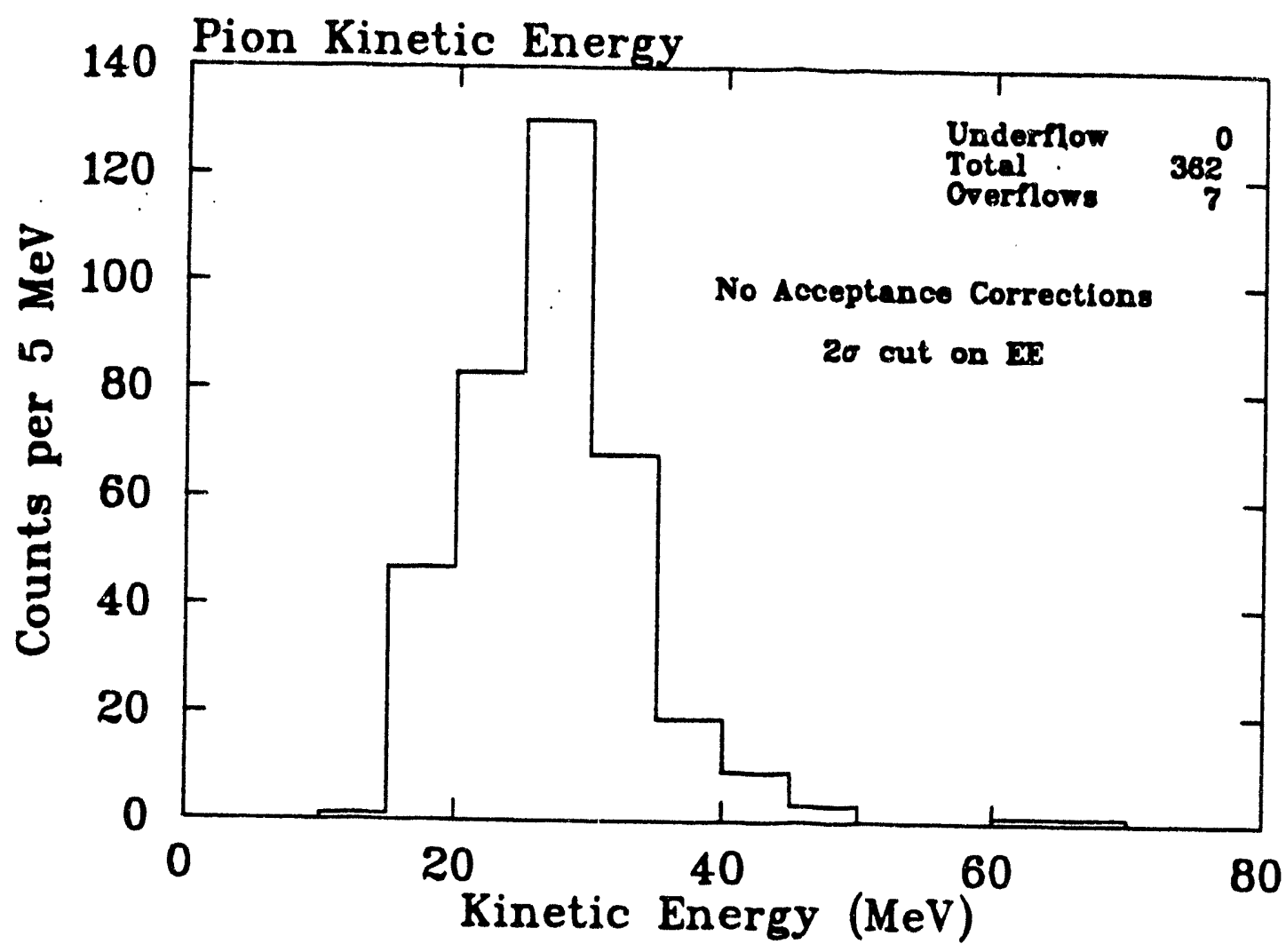

Figure 4.14 A cut is placed on the excitation energy of the pion coincident sample. The resulting kinetic energy distribution for this subsample is shown. No acceptance corrections are made at this point.

\section{Interpretation and Final Extraction}

At this point, some kind of model must be employed to extrapolate the shape of the pion kinetic energy distribution below the low exergy acceptance cut off at about $20 \mathrm{MeV}$.

A simple model is developed in which the $\Lambda$ decay in the nucleus is quasi-free.

- The momentum of the on-shell $\Lambda$ is derived from a momentum distribution desuribed in section 4.2.2. The $\Lambda$ subsequently decays to a $N \pi$. The total energy of the residual nucleus is required to be at least that of ${ }^{4} \mathrm{Li}$. The resulting distribution is smeared according to the predicted kinetic energy resolution. This resolution is a function of the particle's kinetic energy. The resulting kinetic energy distribution of the model is compared to the acceptance corrected kinetic energy distribution in figure 4.15. The 


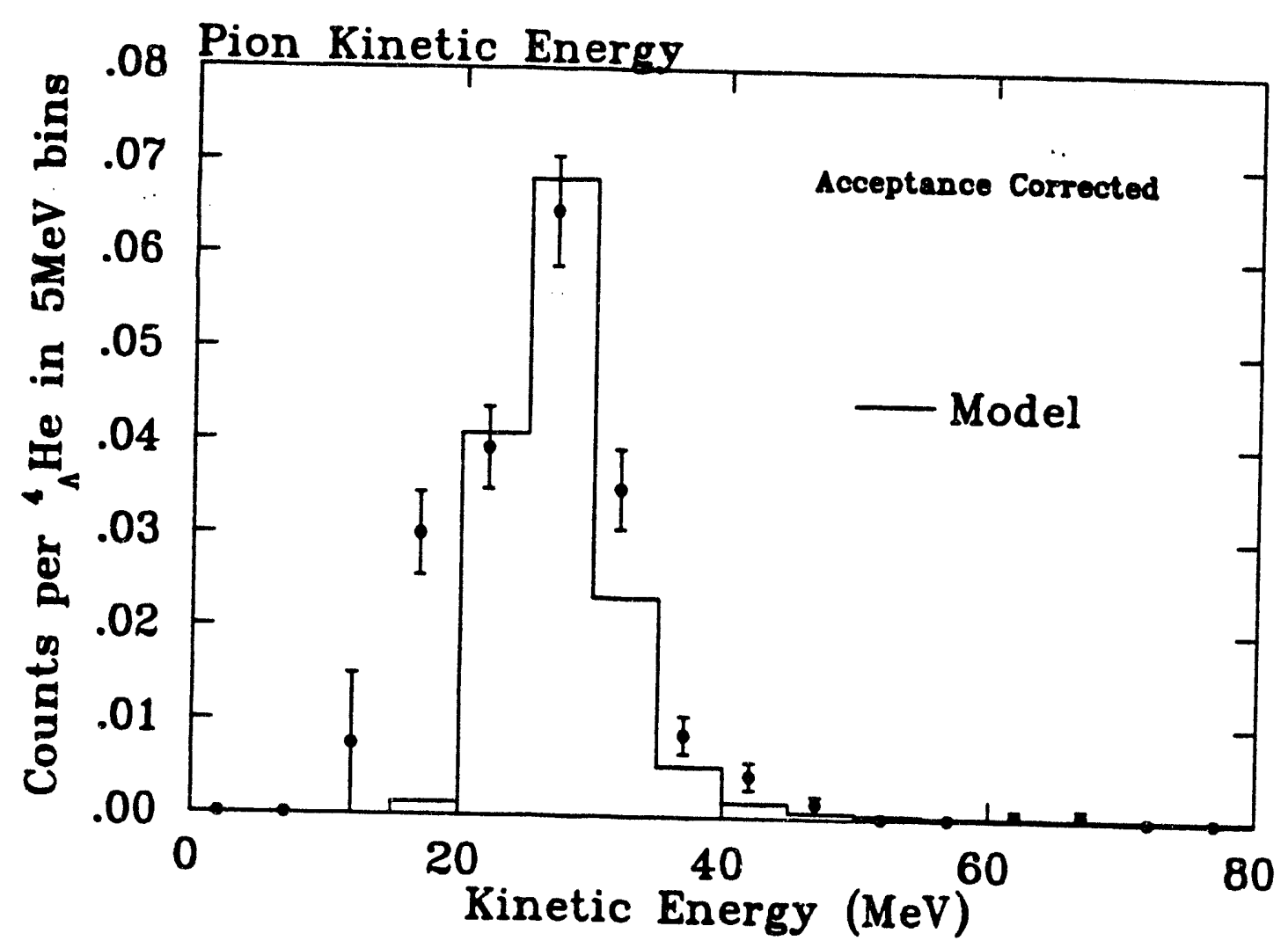

Figure 4.15 The acceptance corrected kinetic energy distribution for pions from hypernuclear decay. The distribution is normalized to the total number of hypernuclei produced. The kinetic energy of the emitted pion in mesonic hypernuclear decay is modeled as the quasi-free decay of a $\Lambda$.

modeled shape is then fit to the data. The total number of pions is then determined from the integral of the normalized shape, $N_{\pi}^{\text {mod }}=2217 \pm 105$. From this, the total number of $\pi^{-}$'s from the decay of ${ }_{A}^{1} \mathrm{He}$ is then

$$
N_{\pi}^{c o r}=\frac{N_{\pi}^{c e}}{N_{\pi}^{2 \sigma}} N_{\pi}^{\text {mod }}=2137 \pm 198 .
$$

\subsubsection{Neutron Measurement}

The neutron signal extraction is treated somewhat differently from the charged particle case since the background rate is much larger. A neutron candidate event is one for which there is a hit in the neutron detector array consistent with a neutron along 


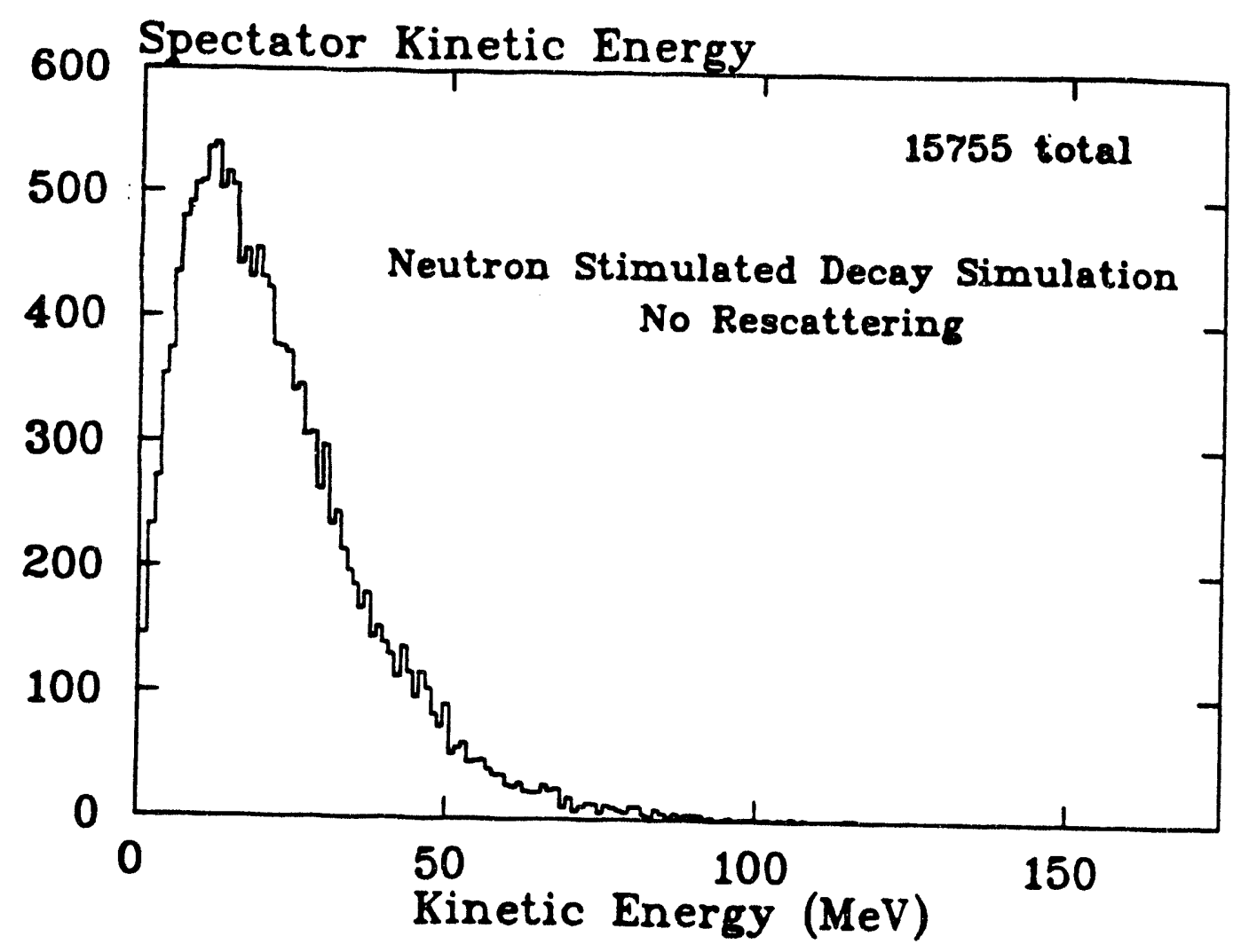

Figure 4.16 The kinetic energy distribution for 25000 generated neutron stimulated decay events is shown. The final state interactions were inhibited for the Monte Carlo.

with various requirements from the target charged particle veto. The details of these requirements will be addressed in this section.

For neutron stimulated decay, $\Lambda+n \rightarrow n+n$, the role of the spectator protons may be relevant for this measurement. The simple model for stimulated decay (described in section 4.2.2) predicts a fraction of spectator protons to have enough energy to escape the ${ }^{4} \mathrm{He}$ target. In this simulation, all final state interactions were switched off - for the generation of 25000 events. Figure 4.16 illustrates the resulting kinetic energy of particles which penetrated the target vessel. The 15755 counts in this distribution indicates that protons are emitted $63 \%$ of the time.

Since it is possible to get energetic protons from neutron stimulated decay, even without considering final state interactions, the role of the charged particle target veto may be defeated. In this analysis, the measurement is actually made without 
the target vetoes.

\section{Data Extraction}

A sample of potential neutron candidates is constructed, in inany ways, in the same manner as the proton sample. Some of the requirements include:

- Quality in-beam tracks are selected where (a) a kaon is identified incident upon the ${ }^{4} \mathrm{He}$ target, (b) kaon decays are removed, and (c) events with poor tracking residuals are eliminated.

- Each event is required to have one or two neutrons, zero to two protons, and zero pions.

Some additional cuts placed upon the data include

- A valid hit in the neutron detector is one for which the TDC information is valid for both photomultipliers. Also, the energy deposition must be above 5 $\mathrm{MeV}$.

- The kinetic energy, as measured by time of flight, must be below $200 \mathrm{MeV}$. This cutoff energy is well above the expected maximum from stimulated decay.

- No restrictions are made based upon the response of the target vetoes.

The resulting excitation energy spectrum for these events is shown in figure 4.17. Despite the restrictions made upon the data, the spectrum is dominated by background.

To understand the relation between the signal and background, the neutron candidate sample (figure 4.17) is decomposed into its various possible sources. Since the $\left(K^{-}, \pi^{-}\right)$production interaction is decoupled from the decay reaction, a grand division can be constructed between production and decay. The possible processes which could happen for the production include true $\left(\mathrm{K}^{-}, \pi^{-}\right)$processes as well as fake $\left(K^{-}, \pi^{-}\right)$processes; that is, processes which satisfy the $\left(K^{-}, \pi^{-}\right)$trigger but are not associated with the $\left(K^{-}, \pi^{-}\right)$reaction.

In general, there are three possible sources which result in a neutral decay trigger. They include; 1) real neutrons, 2) accidentals, 3) $\gamma^{\prime}$ s from $\pi^{\circ}$ decay. When these decay 


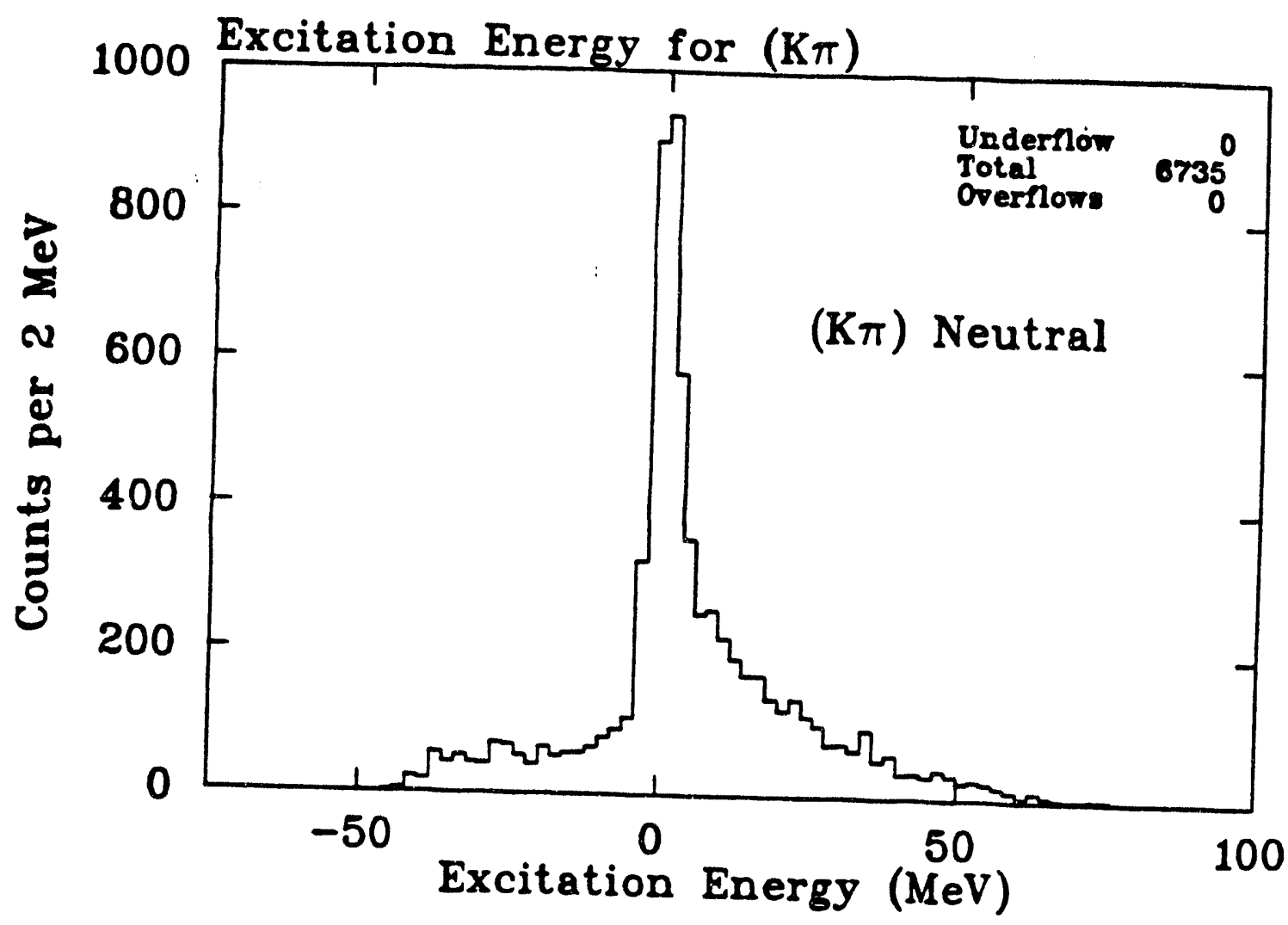

Figure 4.17 The $K \pi N$ excitation energy spectrum for those events for potential neutron candidates. A neutron candidate is an event which has an neutral hit in the neutron detector array with an energy deposition above $5 \mathrm{MeV}$. The hypernuclear formation peak is evident above a large background.

sources are combined with the production sources, the result is six terms present for the neutral candidate sample. The decomposition of the excitation energy for the $K \pi N$ neutral sample can be expressed as:

$$
\begin{aligned}
N_{K \pi N}^{\text {ee }}(E) & =A_{1} f_{(K \pi) n}(E)+A_{2} f_{(K \pi) \gamma}(E)+A_{3} f_{(K \pi) \cdot A c c}(E) \\
& +A_{1} f_{(K \pi)_{\text {gene }} \cdot n}(E)+A_{5} f_{(K \pi)_{\text {gake }} \gamma}(E)+A_{6} f_{(K \pi)_{\text {gahe }} \cdot \text { Ace }}(E)
\end{aligned}
$$

where $A_{i}$ is the amplitude for the normalized distribution $f_{x}$ for source process $x$. In this analysis, we wish to determine the quantity $A_{1}$.

Some of these contributions are easily removed by imposing some simple requirements on the data. Restricting the sample so that events of kinetic energy greater 


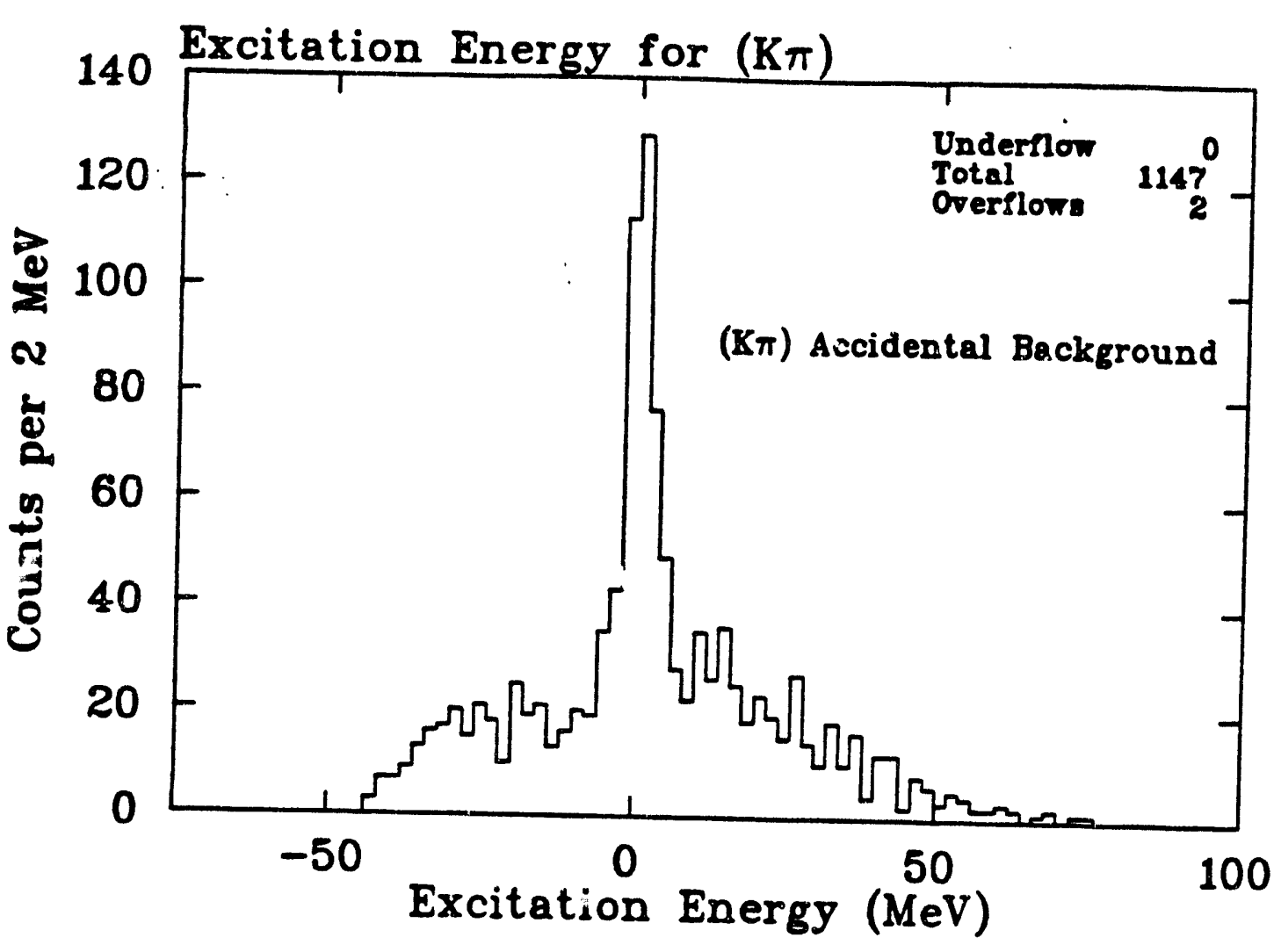

Figure 4.18 The excitation energy spectrum for event $\varepsilon$ which satisfied the $\left(K^{-}, \pi^{-}\right)$trigger in coincidence with a neutral hit in the neutron detector array with energy deposition above $5 \mathrm{MeV}$. These events have a measured kinetic energy (as measured by time-of-flight) below $5 \mathrm{MeV}$ and correspond to a random accidental.

than $200 \mathrm{MeV}$ eliminates terms with photons: $A_{2} f_{(K \pi) \gamma}(E)+A_{5} f_{(K \pi)_{\text {jake }} \gamma}(E)$.

The shape of excitation energy spectrum for accidentals is constructed by extracting events from the $K \pi N$ spectrum which have a kinetic energy below the software threshold of $5 \mathrm{MeV}$ (the kinetic energy is measured by the flight time; long flight times correspond to small kinetic energies): The source of accidental hits include ambient background neutrons from the AGS, cosmic rays, and random photomultiplier tube noise. The excitation energy distribution, $A_{3} f_{(K \pi) \cdot a c c}(E)+A_{6} f_{(K \pi)_{\text {fake }} \text {-ace }}(E)+$

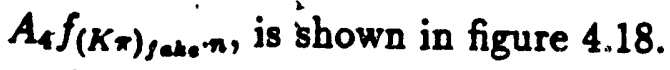

The excitation energy spectrum for accidentals is normalized to the $K \pi N$ spectrum in the region between $-40 \mathrm{MeV}$ to $-5 \mathrm{MeV}$. After we take the difference of the two spectra, the terms in equation (4.24) with Acc are eliminated. What remains is 


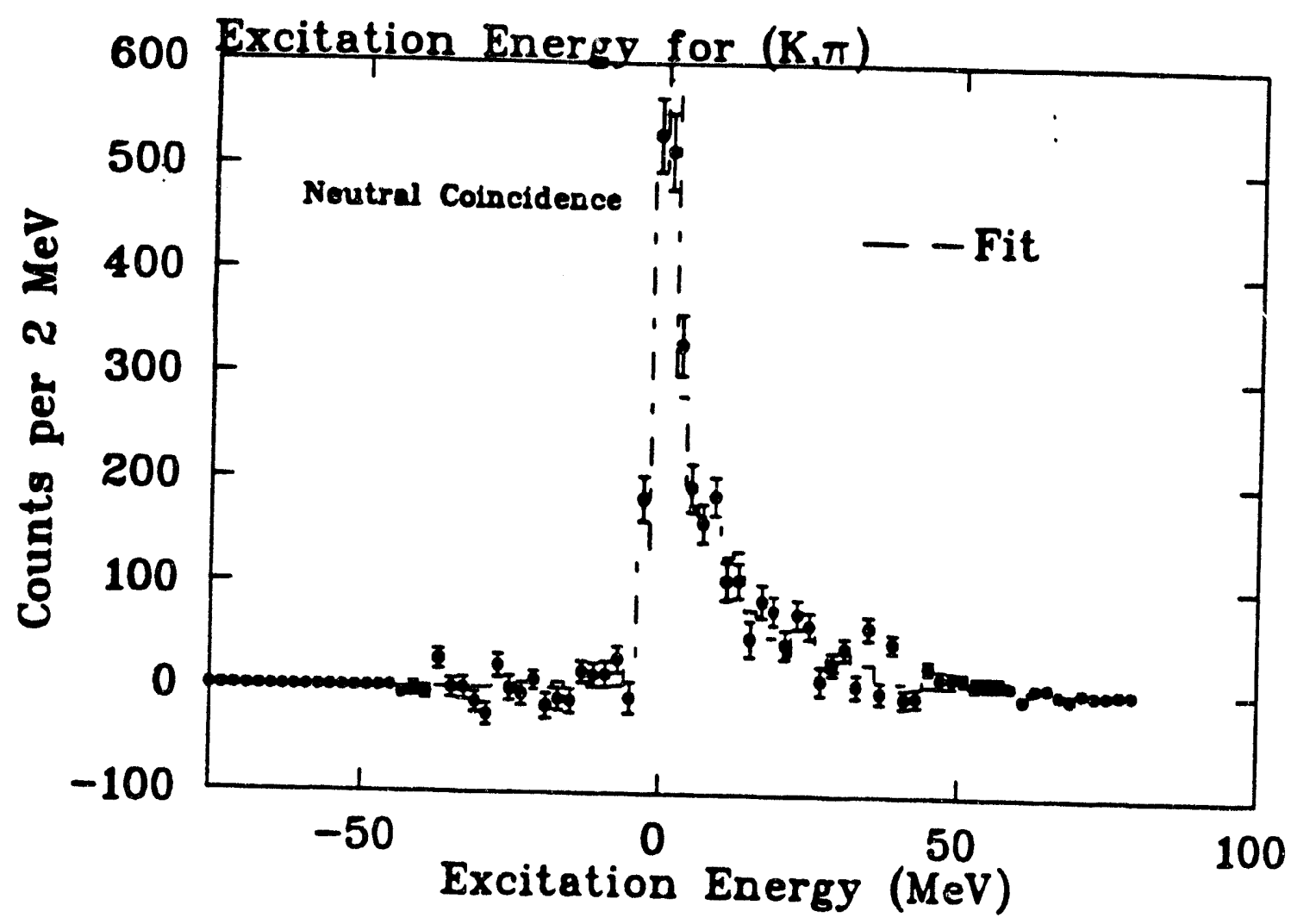

Figure 4.10 The excitation energy spectrum for neutron candidates after the accidental background has been removed.

$A_{1} f_{(K \pi) \cdot n}(E)$, the signal we are interested in. A plot of the difference between figure 4.17 and renormalized figure 4.18 is shown in figure 4.19.

There are two sources of neutrons left in this data sample: 1) neutrons from stimulated decay, and 2) neutrons from the absorption of $\pi^{-}$(the $\pi^{-}$'s originate from hypernuclear mesonic decay or quasi-free $\Lambda$ decay). Fortunately, we know the shape of these two distributions. We safely assume the stimulated decay neutron shape is that of the proton excitation spectrum (figure 4.9). Therefore, the excitation energy

- for neutron stimulated decay is expected to be Gaussian with characteristics of the resolution distribution. The neutrons from $\pi^{-}$absorption will produce an excitation energy spectrum shape similar to that of the mesonic hypernuclear decay (figure 4.13). A two parameter fit is made to the neutron candidate spectrum (figure 4.19) of a Gaussian with EE resolution characteristics and the normalized fion spectrum shape. The amplitudes of the normalized pion and proton spectra, $N_{n(x)}^{\text {id }}$ and $N_{n}^{\text {ee }}$, 
are variables of the weighted fit. The values for for the amplitudes are

$$
N_{n}^{e e}=461 \pm 55 \quad N_{n(\pi)}^{i d}=2409 \pm 107 .
$$

The error quoted is the standard error of the fit (statistical).

Since it is not possible to differentiate a real neutron event from a background event on a event by event basis, the kinetic energy distribution for isolated neutron event cannot be constructed. However, the distribution can be constructed with knowledge of the background shape. There are two components to the background shape: 1) accidentals and 2) real neutrons from $\pi^{-}$absorption.

The kinetic energy distribution for the accidentals is obtained from extracting events with excitation energy between -30 to $-5 \mathrm{MeV}$ (see figure 4.17). The kinetic energy distribution for these events is shown in figure 4.20. This shape is normalized and used henceforth as the accidental background shape.

The shape of the kinetic energy distribution for neutrons from $\pi^{-}$absorption, $n(\pi)$, is obtained from selecting those neutral events with kinetic energies between 5 and $40 \mathrm{MeV}$. This sample also contains events from random background accidentals. The amount of $n(\pi)$ signal is found from integrating the background subtracted spectrum of figure 4.20. This is found to be $N_{b g}^{n(\pi)}=1316 \pm 63$ out of 2615 events. The accidental background is removed. The remaining shape is normalized and used henceforth as the $n(\pi)$ kinetic energy shape.

To extract the neutron kinetic energy, cuts in excitation energy are placed to extract events between $\mu-2 \sigma$ and $\mu+2 \sigma$ of the distribution shown in figure 4.17. The kinetic energy for this sample is shown in figure 4.21. The total number of events in this distribution is $N_{\text {ntot }}^{2 \sigma}=2681$. Its composition consists of

$$
N_{n t o t}^{2 \sigma}=N_{n}^{2 \sigma}+N_{b g}^{2 \sigma}+N_{n(\pi)}^{2 \sigma} \text {. }
$$

The quantity $N_{n(\pi)}^{2 \sigma}$ is estimated from

$$
\begin{aligned}
N_{n(\pi)}^{2 \sigma} & =\frac{N_{\pi}^{2 \sigma}}{N_{\pi}^{i d}} N_{n(\pi)}^{i d} \\
& =982 \pm 71 .
\end{aligned}
$$

The number of neutron events inside this region is estimated from

$$
N_{n}^{2 \sigma}=N_{n}^{e e} \int_{-2 \sigma}^{2 \sigma} d x \frac{1}{\sigma \sqrt{2 \pi}} \exp \left(\frac{-x^{2}}{2 \sigma^{2}}\right)
$$



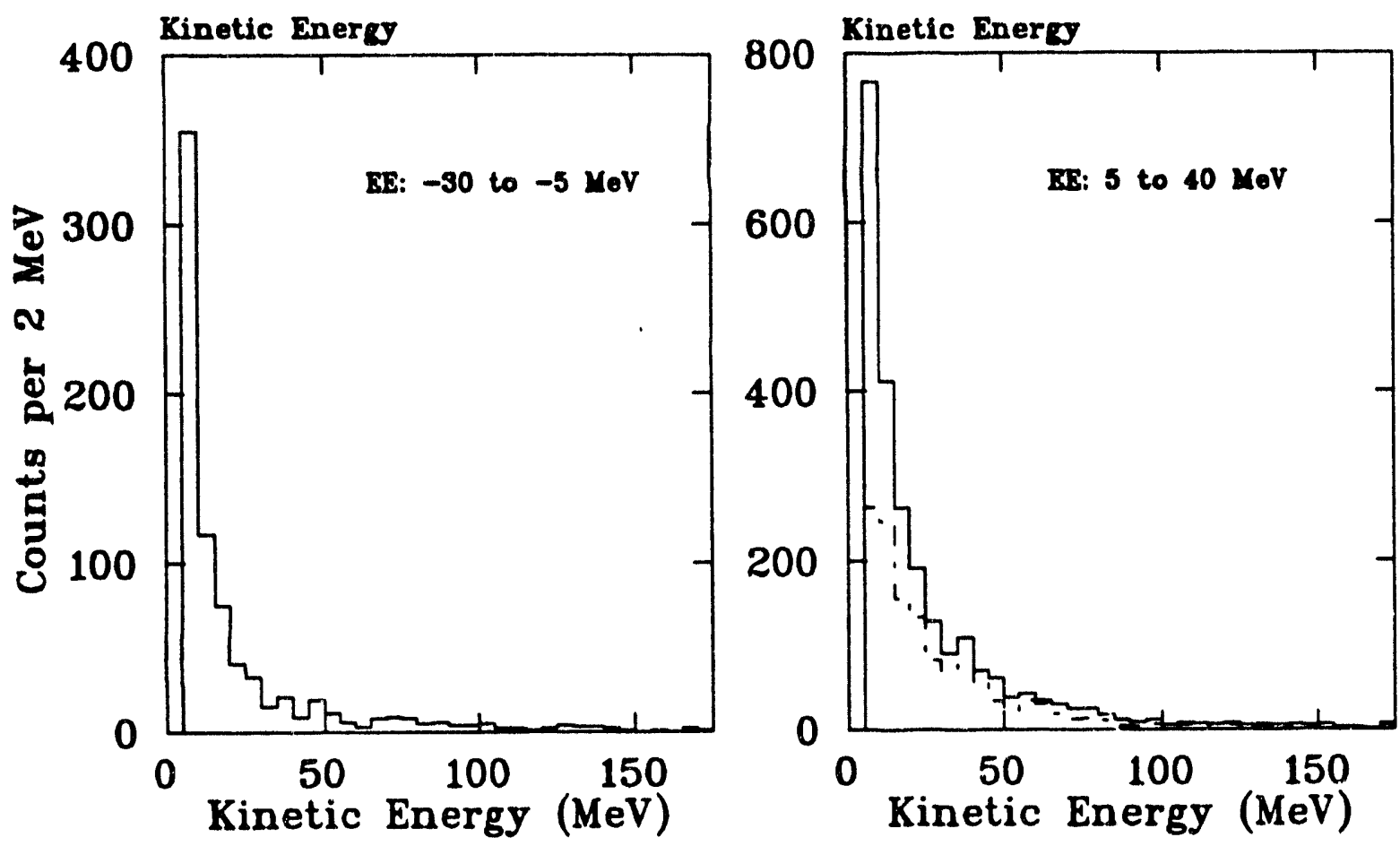

Figure 4.20 LEFT: A cut is placed upon the neutral tag excitation energy spectrum between -30 to $-5 \mathrm{MeV}$ to select background accidentals. The kinetic energy of the tags is shown. RIGHT: An excitation energy cut is placed between 5 and $40 \mathrm{MeV}$ to select events with both accidentals and neutrons from $\pi^{-}$absorption. The dotted line is after the accidental background is subtracted.

$$
=0.95 \times N_{n}^{e e}=437 \pm 52 .
$$

From the above quantities and equation (4.26), the quantity $N_{b g}^{2 \sigma}$ can be inferred. With the aid of these amplitudes, the background of the kinetic energy distribution is isolated (figure 4.21).

For completeness, the neutrons observed in coincidence with a proton tag must also be included in the count. The kinetic energy distribution for these coincident events is shown in figure 4.22. The accidental background contamination of this distribution is estimated as the integral of the flat background term (section 4.2.2). The background is subtracted in the same manner as done previously with the $K \pi N$ 


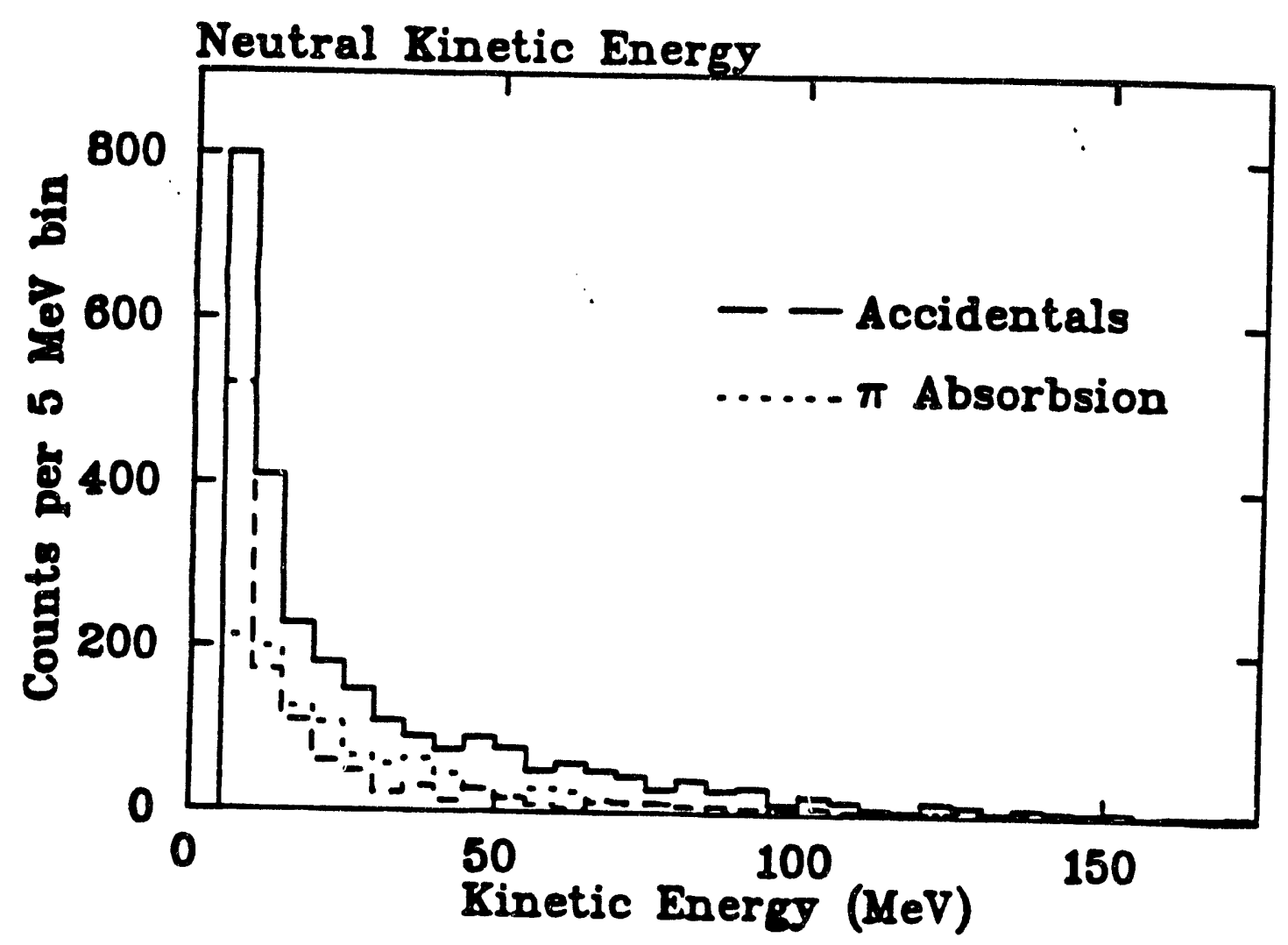

Figure 4.21 The kinetic energy distribution for neutral events with excitar tion energies between $\mu-2 \sigma$ and $\mu+2 \sigma$ is shown. Also shown are the contam. inations from background accidentals and neutrons from $\pi^{-}$absorption.

case. The number of neutrons in the corrected distribution is $N_{n(p n)}^{2 c}=100 \pm 11$. This implies the number of neutrons from hypernuclear decay to be

$$
N_{n(p n)}^{e e}=N_{n(p n)}^{2 \sigma} \frac{N_{p}^{e e}}{N_{p}^{2 \sigma}}=94 \pm 14 .
$$

The final observed neutron kinetic energy distribution with the backgrounds subtracted and neutrons from coincident protons is shown in figure 4.23.

\section{Acceptance Correction}

At this point, acceptance corrections are made in the same manner as in the proton and pion case. The procedure for the bin by bin acceptance correction is described in section 3.5. The acceptance corrected neutron kinetic energy distribution is given 


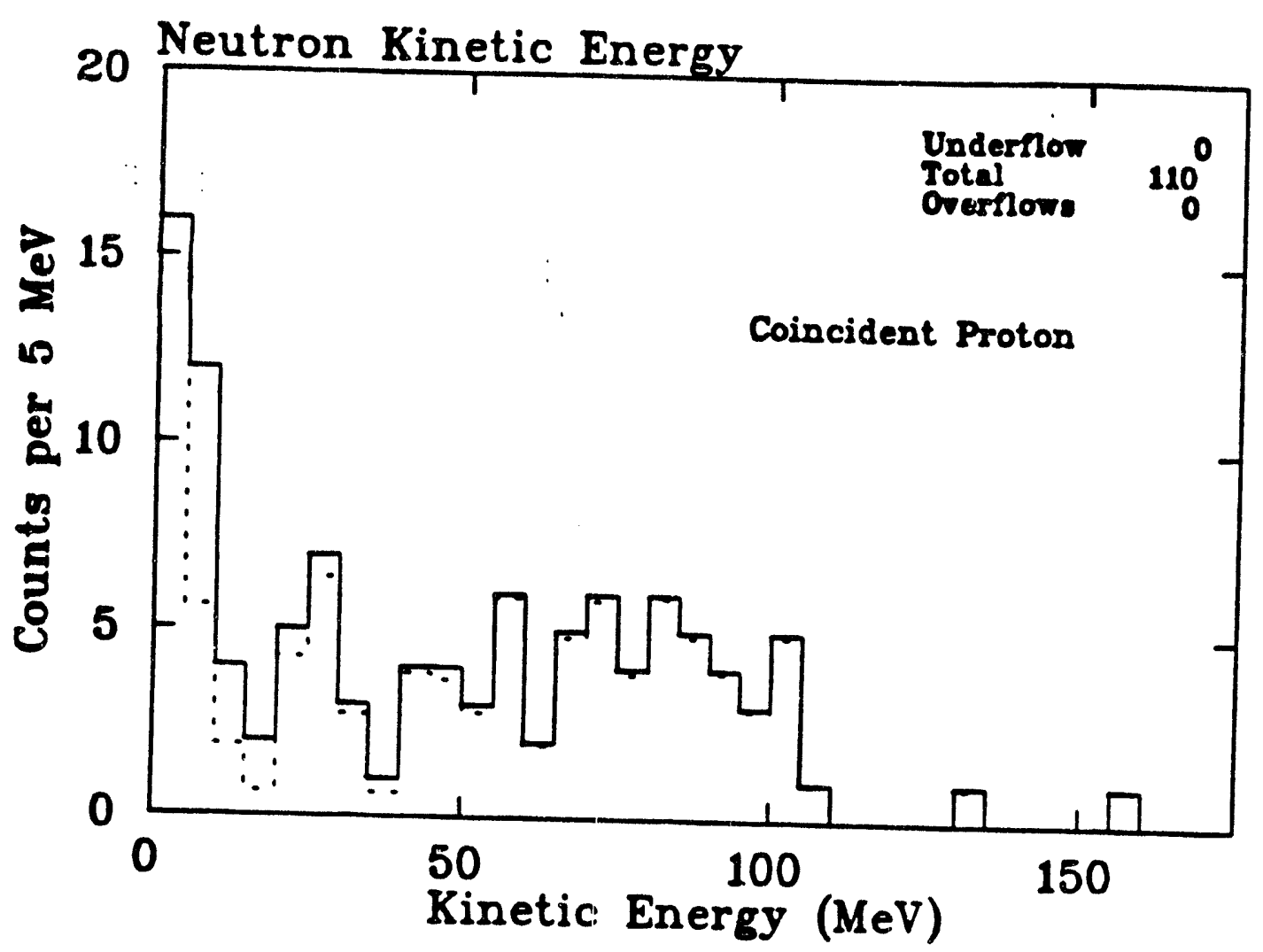

Figure 4.22 The kinetic energy distribution for neutrons in coincidence with a proton from hypernuclear decay is shown. The sample was selected to have $\left(K^{-}, \pi^{-}\right)$excitation energies within $\mu-2 \sigma$ and $\mu+2 \sigma$. The dotted line is with accidental backgrounds subtracted out.

in figure 4.24. The number of acceptance corrected neutrons is determined from integrating the distribution from $10 \mathrm{MeV}$ to $175 \mathrm{Mev}$,

$$
N_{n}=8089 \pm 1034 .
$$

\subsection{Rates and Normalization}

The number of acceptance corrected neutrons (protons) is not the number found from neutron (proton) stimulated decay. As mentioned before, neutrons and protons are emitted for both types of decay; both directly, as in the case of $\Lambda p \rightarrow n p$, and 


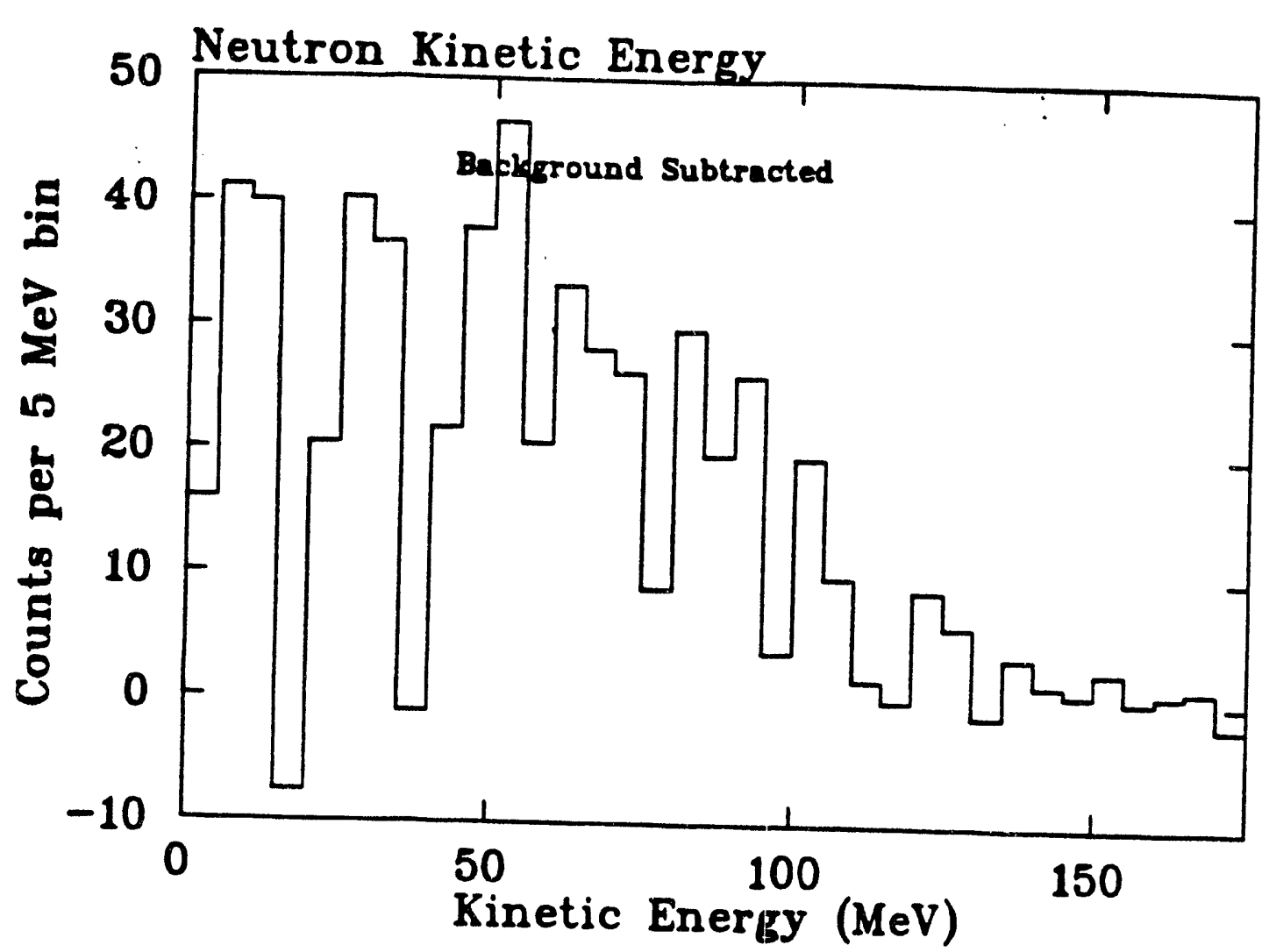

Figure 4.23 The kinetic energy distribation for neutrons from stimulated decay is shown. Backgrounds from random accidentals and neutrons from $\pi^{-}$absorption have been subtracted. Also, observed neutrons in coincidence with a proton are included.

as a result of final state interactions. An adjustment is made for these effects by decomposing the various possibilities of the final states. Unfortunately, the process of untangling the various probabilities can easily get confusing. To begin, we define the following quantities:

- $\epsilon$, the probability that the a final state nucleon from $\Lambda N \rightarrow N N$ will interact with a residual nucleon (see table 4.3).

- $\alpha$, the probability that the final state nucleons from stimulated decay are emitted from the nucleus without final state interactions. 


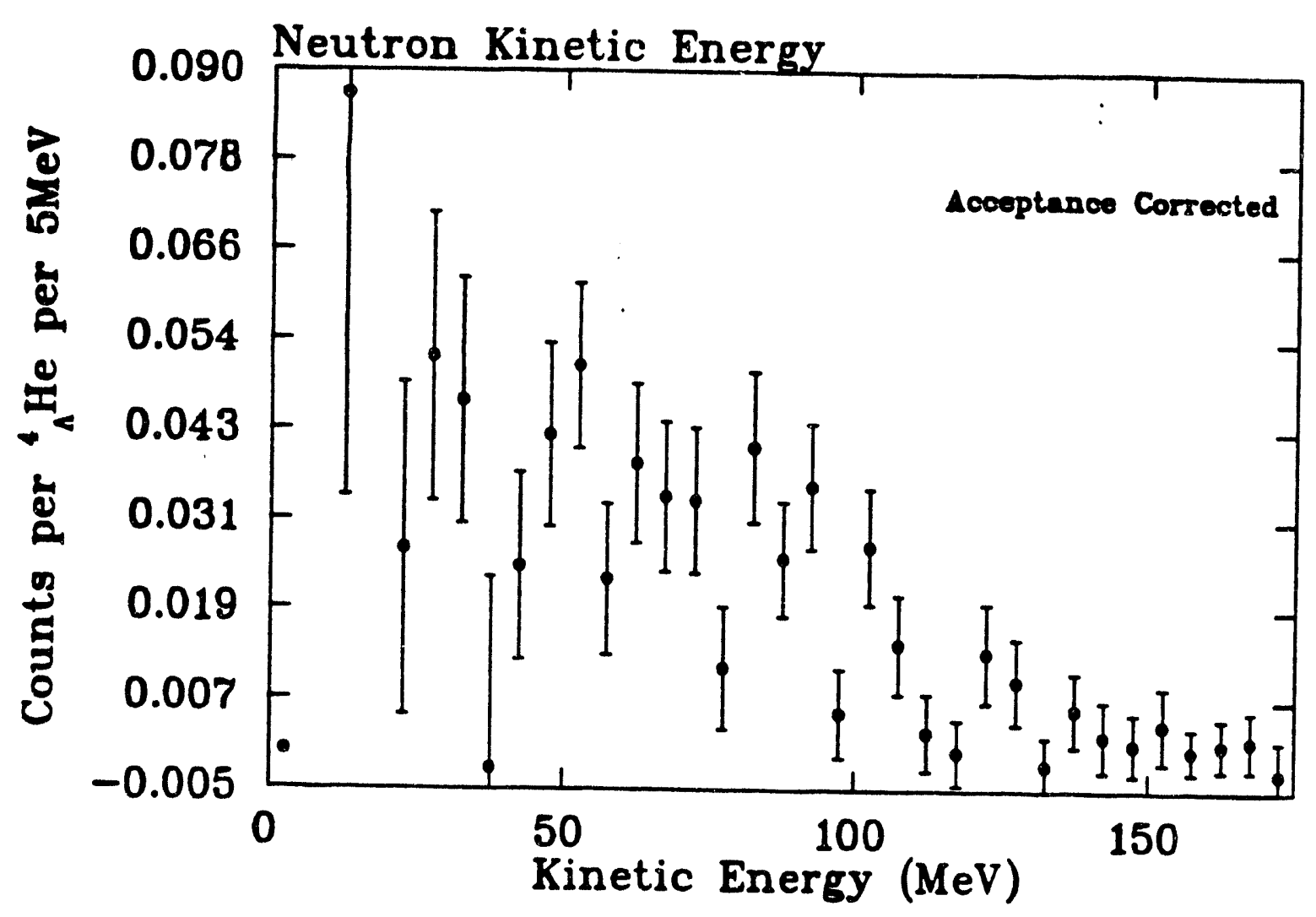

Figure 4.24 The acceptance corrected neutron kinetic energy distribution.

- $N_{p}$, the total number of protons observed in the course of the experiment. This has a value $N_{p}=N_{p}^{\text {cor }}$ (see equation (4.21)).

- $N_{n}$, the total number of neutrons observed in the course of the experiment. This has a value $N_{n}=N_{n}^{\text {cor }}$ (see equation (4.30)).

- $P_{N}$, the probability that $N$ stimulated decay take place $(N=n$ or $p)$.

- $M_{T}$, the total number of stimulated decay events. Note, $M_{T} \leq N_{p}+N_{n}$, that is, the total number of events observed during the experiment is less than or equal to the total number of protons and neutrons observed. This is because each event can have more that one proton and/or neutron.

- $\rho_{i}$, the proton multiplicity of process ID $i$. 


\begin{tabular}{|c|c|c|c|c|}
\hline \multicolumn{5}{|c}{ Stimulated Decay Final States } \\
\hline \hline ID & Process & Process Probability $\mathcal{P}_{i}$ & $\rho_{i}$ & $\eta_{i}$ \\
\hline 1 & $\Lambda p \rightarrow n p$ & $\alpha P_{p}$ & 1 & 1 \\
\hline 2 & $\Lambda n \rightarrow n n$ & $\alpha P_{n}$ & 0 & 2 \\
\hline 3 & $\Lambda p \rightarrow n(p p)$ & $\epsilon P_{p}$ & 2 & 1 \\
\hline 4 & $\Lambda p \rightarrow n(p n)$ & $\epsilon P_{p}$ & 1 & 2 \\
\hline 5 & $\Lambda p \rightarrow p(n n)$ & $\epsilon P_{p}$ & 1 & 2 \\
\hline 6 & $\Lambda p \rightarrow p(n p)$ & $\epsilon P_{p}$ & 2 & 1 \\
\hline 7 & $\Lambda n \rightarrow n(n p)$ & $4 \epsilon P_{n}$ & 1 & 2 \\
\hline
\end{tabular}

Table 4.3 The final state stimulated decay nucleons may either be emitted from the nucleus without interactions or undergo interactions with the residual nucleons. The possible processes are shown in the first column. The nucleons in parentheses interact.

- $\eta_{i}$, the neutron multiplicity of process ID $i$.

The hypernuclear decay can proceed with or without final state interactions. The possible processes which can subsequently happen after a stimulated decay are tabulated in table 4.3.

The list of final state possibilities given in table 4.3 is complete (we are ignoring three and four body interactions). Some rudimentary rules are established from these relations. Either proton stimulated decay or neutron stimulated decay can take place. These are the only alternatives. Therefore,

$$
P_{p}+P_{n}=1
$$

Since the processes listed in 4.3 are complete, the sum of probabilities $\mathcal{P}_{i}$ must equal 1. This leads to the rule;

$$
\sum_{i} \mathcal{P}_{i}=1=\alpha+4 \epsilon
$$

The various processes listed in táble 4.3 have different chances of being detected depending upon the number of nucleons in the final state. For example, the detection of protons from process $\mathrm{ID}=3$ is twice as likely than process $\mathrm{ID}=4$ since it has two 
protons in the final state compared to the one proton in process $I D=4$ (the spectator nucleons are ignored). The multiplicity for protons (neutrons) in the final state for process $i$ is denoted as $\rho_{i}\left(\eta_{i}\right)$ and is also listed in the table 4.3. The expectation number of protons from process $i$ per event is $\mathcal{P}_{i} \rho_{i}$. Carrying this one step further, the total number of protons emitted $N_{p}$ is related to the total number of events $M_{t}$ by

$$
N_{p}=M_{t} \sum_{i=1}^{7} \mathcal{P}_{i} \rho_{i}
$$

Similarly, the number of neutrons is determined from

$$
N_{n}=M_{t} \sum_{i=1}^{7} \mathcal{P}_{i} \eta_{i}
$$

These equations are expanded to yield;

$$
\begin{gathered}
N_{p}=M_{t}\left[\alpha P_{p}+\epsilon\left(6 P_{p}+4 P_{n}\right)\right], \\
N_{n}=M_{t}\left[\alpha\left(P_{p}+2 P_{n}\right)+\epsilon\left(6 P_{p}+8 P_{n}\right)\right] .
\end{gathered}
$$

The quantity $M_{t}$ is difficult to pin down. It is not possible to derive the total number of events produced in the experiment since the number of efficiency corrected neutron and proton events are inter-related. That is, efficiency corrected neutrons may correspond to efficiency corrected protons. For this reason, $M_{t}$ is left as a floating parameter and is not extracted directly from data.

The number of observed protons $N_{p}$ can also be related to the amplitudes measured for no-scatter and one-scatter fits (equations (4.20)). The quantity $N_{1 \varepsilon}$ is related to equation (4.35) by

$$
N_{1 s}=M_{t} \epsilon\left(6 P_{p}+8 P_{n}\right) .
$$

It is now possible to extract the probabilities for proton and neutron stimulated decays, $P_{p}$ and $P_{n}$ from the above equations. There are five equations $((4.31),(4.32)$, (4.33), (4.34), and (4.37) ) and 5 unknowns ( $\alpha, \epsilon, P_{p}, P_{n}$, and $\left.M_{t}\right)$. The resulting

$=$ value for $P_{p}$ and error analysis is derived with the assistance of a mathematical symbol manipulation program, MAPLE. The expression for the probability for proton stimulated decay is

$$
\begin{aligned}
P_{p} & =\frac{1}{2 M_{t}}\left(N_{p}+N_{1,}-2 M_{t}\right. \\
& \left.+\left[N_{p}^{2}+2 N_{p} N_{1}+4 N_{p} M_{t}+N_{1 \vartheta}^{2}-12 N_{1}, M_{t}+4 M_{t}^{2}\right]^{\frac{1}{2}}\right) .
\end{aligned}
$$


The end result yields

$$
\begin{aligned}
P_{p} & =0.466 \pm 0.092 \\
M_{t} & =5653 \pm 569 \\
\epsilon & =0.039 \pm 0.0087 .
\end{aligned}
$$

The stimulated decay rates, $S_{N}$, are

$$
\begin{aligned}
& S_{p}=P_{p} M_{t}=2636 \pm 518 \\
& S_{n}=P_{n} M_{t}=3017 \pm 590 .
\end{aligned}
$$

\subsection{Partial Rates}

As expressed in the outset of this chapter, the partial decay rates are related to the various measurements by equation (4.13). However, previous decay rates are measured in terms of the known decay rate of free $\Lambda$ 's:

$$
\Gamma_{i}=\frac{N_{i}}{N_{t o t}} \frac{\Gamma_{t o t}}{\Gamma_{\Lambda}} \Gamma_{\Lambda}
$$

A summary of the various decay rates is given in table 4.4 . 


\begin{tabular}{|c|c|}
\hline \multicolumn{2}{|c|}{ Measurements of ${ }_{\Lambda}^{1} \mathrm{He}$ Decay } \\
\hline \hline Quantity & Value \\
\hline lifetime & $245_{-23}^{+24} \mathrm{ps}$ \\
\hline$\Gamma_{p} / \Gamma_{\Lambda}$ & $0.226 \pm 0.052$ \\
\hline$\Gamma_{n} / \Gamma_{\Lambda}$ & $0.259 \pm 0.060$ \\
\hline$\Gamma_{\pi-} / \Gamma_{\Lambda}$ & $0.176 \pm 0.026$ \\
\hline$\Gamma_{\pi \circ} / \Gamma_{\Lambda}$ & $0.41 \pm 0.12$ \\
\hline$\frac{\Gamma_{n}}{\Gamma_{n}+\Gamma_{p}}$ & $0.363 \pm 0.080$ \\
\hline$\frac{\Gamma_{n}}{\Gamma_{n}+\Gamma_{p}}$ & $0.534 \pm 0.081$ \\
\hline$\frac{\Gamma_{n}}{\Gamma_{p}}$ & $1.14 \pm 0.37$ \\
\hline
\end{tabular}

Table 4.4 Measurements of the decay of ${ }_{\Lambda}^{4} \mathrm{He}$ - this experiment. $\Gamma_{\Lambda}$ is the free $\Lambda$ decay rate. 


\section{DISCUSSION AND CONCLUSION}

An overview of all measurements made for the decay of ${ }_{\Lambda}^{4} \mathrm{He}$ are reviewed in this chapter. A comparison to measurements made on other light hypernuclei is also established. We also investigate the bearing of this measurement to the isospin amplitude for baryon-baryon weak interactions.

\subsection{General Discussion}

A summary of all ${ }_{\Lambda}^{4} \mathrm{He}$ hypernuclear decay rates is presented in table 4.4. A summary of the important quantities used to derive the proton, pion, neutron, and total rates are presented in tables 5.1-4.

\subsubsection{Comparison to Previous Results}

As mentioned in the first chapter, there are very few quantitative results for the decay of ${ }_{\Lambda}^{4} \mathrm{He}$. The summary of previous measurements is presented in table 1.2. Of the sparse previous data available, there is a measurement for the ${ }_{\Lambda}^{4} \mathrm{He}$ hypernuclear lifetime made by Phillips in 1969 . The error quoted on this quantity is rather large and is thus consistent with our result.

Block et al.[14] made measurements of $\frac{\Gamma_{n}}{\Gamma_{n}+\Gamma_{p}}$ and $\frac{\Gamma_{n}}{\Gamma_{n}+\Gamma_{p}}$. The measurements were made using a ${ }^{4} \mathrm{He}$ bubble chamber. A total of 28 hypernuclei were isolated to ascertain these values. This first measurement differs from ours by $4.7 \sigma$. The later 
measurement is about $2.9 \sigma$ from our result.

A comparison can also be made to a similar hypernucleus, ${ }_{\Lambda}^{5} \mathrm{He}$. The measurement was performed by John Szymanski et al.[8] at BNL during the mid 1980's. A solid ${ }^{6} \mathrm{Li}$ target is used to produce the unstable hypernucleus ${ }_{\Lambda}^{6} \mathrm{Li}$, which decays strongly to ${ }_{\Lambda}^{5} \mathrm{He}$. A summary of the rate measurements for this experiment is given in table 5.5 (compare with table 4.4).

The $\pi^{-}$decay rates $\Gamma_{\pi}$ differ for the two experiments. For the present analysis, we identified a large number of pions, $N_{\pi}^{i d}$. However, only $N_{\pi}^{e e}$ were determined to have originated from hypernuclear decay. It may not have been possible to make a distinction between pions produced from quasi-free $\Lambda$ decay for the ${ }_{\Lambda}^{5} \mathrm{He}$ measurement because of the lower excitation energy resolution inherent to dense targets. A good energy resolution may have been lacking for the ${ }_{\Lambda}^{5} \mathrm{He}$ experiment.

We would also naively expect the rate $\Gamma_{\pi^{\circ}}$ to be enhanced for the ${ }_{\Lambda}^{4} \mathrm{He}$ case compared to ${ }_{\Lambda}^{5} \mathrm{He}$. This is because the decay neutron from $\Lambda \rightarrow n+\pi^{\circ}$ is not blocked from occupying a filled orbital as in the ${ }_{\Lambda}^{5} \mathrm{He}$ case.

\subsubsection{Implications for the $\Delta I=\frac{1}{2}$ Rule}

As outlined in the first chapter, the three quantities, $\gamma_{n m}, \gamma_{n p}^{4}, \gamma_{n p}^{5}$ (see equation (1.26)), can be used to extract the ratio of the neutron to proton stimulated rate for $I_{f}=1$ final states, $\epsilon^{2}=R_{n 0} / R_{p 0}$. A definitive prediction can be made for this ratio at the cost of very few assumptions. The $\Delta I=\frac{1}{2}$ rule predicts a value $\epsilon^{2}=2$.

The present analysis of ${ }_{\Lambda}^{4} \mathrm{He}$ effects both $\gamma_{n m}$ and $\gamma_{n p}^{4}$. Using our current measurement, the up-to-date values are

$$
\begin{aligned}
& \gamma_{n m}=1.79 \pm 0.86 \\
& \gamma_{n p}^{4}=1.14 \pm 0.37 .
\end{aligned}
$$

The $\gamma_{n m}$ value is dependent upon measurements made on ${ }_{\Lambda}^{4} \mathrm{H}$ (see equation (1.28)). In the above calculation, we have used

$$
\tau\left({ }_{\Lambda}^{4} \mathrm{H}\right)=194 \pm 30 p s \quad Q^{-}\left({ }_{\Lambda}^{4} \mathrm{H}\right)=0.26: \pm 0.13 \quad R_{\circ}\left({ }_{\Lambda}^{4} \mathrm{H}\right)=0.108 \pm 0.035
$$

The value of $R_{0}\left({ }_{\Lambda}^{4} \mathrm{H}\right)$ is calculated from ${ }_{\Lambda}^{4} \mathrm{He}$ measurements and assuming the $\Delta I=\frac{1}{2}$ rule. 
The ratio $\epsilon^{2}$ (see equation (1.27)) contains the difference of $\gamma_{i}$ measurements in the denominator. Care must be taken to insure a value of zero is excluded for the denominator. For this reason, we will actually construct the quantity $\epsilon^{-2}$.

The error estimate for this analysis is obtained from

$$
\sigma^{2}=\sum\left(\frac{\partial \epsilon^{-2}}{\partial x} \sigma_{x}\right)^{2}
$$

where $x$ is a quantity obtained from experiment. The $x$ in the above relations refers to the set of measurements:

$$
\begin{aligned}
& \Gamma_{\pi}-\left({ }_{\Lambda}^{4} \mathrm{He}\right), \quad \Gamma_{\pi^{\circ}}\left({ }_{\Lambda}^{4} \mathrm{He}\right), \quad \Gamma_{p}\left({ }_{\Lambda}^{4} \mathrm{He}\right), \quad \Gamma_{n}\left({ }_{\Lambda}^{4} \mathrm{He}\right), \quad \tau\left({ }_{\Lambda}^{4} \mathrm{He}\right), \\
& \Gamma_{p}\left({ }_{\Lambda}^{5} \mathrm{He}\right), \quad \Gamma_{n}\left({ }_{\Lambda}^{5} \mathrm{He}\right), \quad \tau\left({ }_{\Lambda}^{4} \mathrm{H}\right), \quad \text { and } \quad Q^{-}\left({ }_{\Lambda}^{4} \mathrm{H}\right) .
\end{aligned}
$$

The algebra/calculus is handled by the symbolic mathematics package, MAPLE. The results of the calculation is

$$
\epsilon^{-2}=0.25 \pm 0.67
$$

Note, this result is consistent with the $\Delta I=\frac{1}{2}$ rule prediction of $\epsilon^{-2}=\frac{1}{2}$. The magnitude of the error is a result of the relatively large partials from equation (5.4). Contributions to this error are tabulated in table 5.6.

\subsubsection{Future}

If the goal is to investigate the isospin structure of the weak baryon-baryon interaction it may be necessary to make additional measurements of the decay of light hypernuclei. This collaboration made measurements on ${ }_{\Lambda}^{5} \mathrm{He}$ at Brookhaven in the same running period as the ${ }_{\Lambda}^{4} \mathrm{He}$ experiment. Because of a manpower shortage, the data are not expected to be analyzed until the Spring of '93. In this measurement, we expect to make a great improvement as far as the neutron measurement goes.

Much improved measurements of the ${ }_{\Lambda}^{1} \mathrm{H}$ system would also help to achieve our goal. At the moment there are no explicit plans for this measurement by this research group. 


\begin{tabular}{|c|l|c|}
\hline \multicolumn{3}{|c|}{ Summary of Proton Measurement } \\
\hline \hline Quantity & Description & Value \\
\hline$N_{p}^{\text {id }}$ & total number of protons identified & 596 \\
\hline$N_{p}^{e e}$ & $\begin{array}{l}\text { number of protons from hypernuclear de- } \\
\text { cay }\end{array}$ & $456 \pm 24$ \\
\hline$N_{p}^{2 \sigma}$ & $\begin{array}{l}\text { number of identified protons in } 2 \sigma \text { excita- } \\
\text { tion energy cut }\end{array}$ & 483 \\
\hline$N_{0 s}$ & $\begin{array}{l}\text { integrated number of events of a fit to } \\
\text { the modeled proton kinetic energy for no } \\
\text { rescattering }\end{array}$ & $2307 \pm 159$ \\
\hline$N_{1 s}$ & $\begin{array}{l}\text { integrated number of events of a fit to the } \\
\text { modeled proton kinetic energy for rescat- } \\
\text { tering }\end{array}$ & $576 \pm 256$ \\
\hline$N_{p}^{\text {cor }}$ & $\begin{array}{l}\text { integrated number of events of a fit to the } \\
\text { modeled proton kinetic energy distribution }\end{array}$ & $2722 \pm 342$ \\
\hline$\sigma_{e e}$ & $\begin{array}{l}\text { width of hypernuclear excitation energy } \\
\text { hypernuclear ground state peak - taken as } \\
\text { energy resolution }\end{array}$ & $1.86 \pm 0.046 \mathrm{MeV}$ \\
\hline$\mu_{e e}$ & $\begin{array}{l}\text { mean position of hypernuclear excitation } \\
\text { energy hypernuclear ground state peak }\end{array}$ & $0.185 \pm 0.040 \mathrm{MeV}$ \\
\hline
\end{tabular}

Table 5.1 The resulting quantities from the measurement of hypernuclear decay coincident with an identified proton are summarized. 


\section{Summary of Pion Measurement}

\begin{tabular}{|c|l|c|}
\hline \hline Quantity & Description & Value \\
\hline$N_{\pi}^{\text {id }}$ & total number of $\pi^{-}$'s identified & 856 \\
\hline$N_{\pi}^{\text {ee }}$ & number of $\pi^{-}$'s from hypernuclear decay & $349 \pm 21$ \\
\hline$N_{\pi}^{2 \sigma}$ & $\begin{array}{l}\text { number of identified pions in } 2 \sigma \text { excitation } \\
\text { energy cut }\end{array}$ & 362 \\
\hline$N_{\pi}^{\text {mod }}$ & $\begin{array}{l}\text { integrated number of events of a fit to the } \\
\text { modeled pion kinetic energy }\end{array}$ & $2217 \pm 105$ \\
\hline$N_{\pi}^{\text {cor }}$ & $\begin{array}{l}\text { total number of acceptance and efficiency } \\
\text { corrected pions }\end{array}$ & $2137 \pm 198$ \\
\hline
\end{tabular}

Table 5.2 The resulting quantities from the measurement of hypernuclear $\pi^{-}$decay are summarized. 


\begin{tabular}{|c|l|c|}
\hline \multicolumn{3}{|c|}{ Summary of Neutron Measurement } \\
\hline \hline Quantity & Description & Value \\
\hline$N_{n}^{\text {id }}$ & $\begin{array}{l}\text { total number of neutron candidates (no } \\
\text { coincidence) identified, background sub- } \\
\text { tracted }\end{array}$ & 2964 \\
\hline$N_{n}^{e e}$ & $\begin{array}{l}\text { number of neutrons from hypernuclear de- } \\
\text { cay (no coincidence) }\end{array}$ & $437 \pm 52$ \\
\hline$N_{n n}^{2 \sigma}$ & $\begin{array}{l}\text { number of identified neutrons in } 2 \sigma \text { excita- } \\
\text { tion energy cut (no coincidence) }\end{array}$ & $461 \pm 55$ \\
\hline$N_{n p}^{e e}$ & $\begin{array}{l}\text { number of neutrons from hypernuclear de- } \\
\text { cay (with proton coincidence) with acci- } \\
\text { dental background removed }\end{array}$ & $94 \pm 14$ \\
\hline$N_{n p}^{2 \sigma}$ & $\begin{array}{l}\text { number of identified neutrons in } 2 \sigma \text { excita- } \\
\text { tion energy cut (with proton coincidence) } \\
\text { with accidental background removed }\end{array}$ & $100 \pm 11$ \\
\hline$N_{n}^{\text {cor }}$ & $\begin{array}{l}\text { integrated number of events from accep- } \\
\text { tance and efficency corrected neutron ki- } \\
\text { netic distribution between 10 to 175 MeV }\end{array}$ & $8089 \pm 1034$ \\
\hline
\end{tabular}

Table 5.3 The resulting quantities from the measurement of hypernuclear decay coincident with an identified neutron are summarized. 


\begin{tabular}{|c|l|c|}
\hline \multicolumn{2}{|c|}{ Summary of Hyp rnuclear Rate Measurements } \\
\hline \hline Quantity & Description & Value \\
\hline$\tau$ & hypernuclear lifetime & $245_{-23}^{+24} \mathrm{ps}$ \\
\hline$N_{K \pi}$ & $\begin{array}{l}\text { total prescaled observed hypernuclear } \\
\text { events }\end{array}$ & $626 \pm 46$ \\
\hline$N_{K \pi}^{\text {tot }}$ & $\begin{array}{l}\text { total number of hypernuclear produced in } \\
\text { the course of the experiment }\end{array}$ & $12520 \pm 922$ \\
\hline
\end{tabular}

Table 5.4 The resulting quantities from the measurement of the total hypernuclear decay rate.

\begin{tabular}{|c|c|}
\hline \multicolumn{2}{|c|}{ Measurements of ${ }_{\Lambda}^{5} \mathrm{He}$ Decay } \\
\hline \hline Quantity & Value \\
\hline lifetime & $256 \pm 20 \mathrm{ps}$ \\
\hline$\Gamma_{p} / \Gamma_{\Lambda}$ & $0.21 \pm 0.07$ \\
\hline$\Gamma_{n} / \Gamma_{\Lambda}$ & $0.20 \pm 0.11$ \\
\hline$\Gamma_{\pi} / \Gamma_{\Lambda}$ & $0.44 \pm 0.11$ \\
\hline$\Gamma_{\pi^{\circ}} / \Gamma_{\Lambda}$ & $0.18 \pm 0.20$ \\
\hline$\frac{\Gamma_{n}+\Gamma_{p}}{\Gamma_{\ulcorner-}}$ & $0.92 \pm 0.31$ \\
\hline$\frac{\Gamma_{n}}{\Gamma_{p}}$ & $0.93 \pm 0.55$ \\
\hline
\end{tabular}

Table 5.5 Measurements of the decay of ${ }_{\Lambda}^{5} \mathrm{He}$ [24]. $\Gamma_{\Lambda}$ is the free $\Lambda$ decay rate. 


\begin{tabular}{|c|c|c|}
\hline \multicolumn{3}{|c|}{ Error Contributions } \\
\hline \hline$x$ & $\sigma_{i}^{2}\left(\frac{\partial \epsilon^{-2}}{\partial x}\right)^{2}$ & $\frac{\partial \epsilon^{-2}}{\partial x}$ \\
\hline \hline$\Gamma_{\pi-}\left({ }_{\Lambda}^{4} \mathrm{He}\right)$ & 0.00019 & 0.50 \\
\hline$\Gamma_{\pi^{\circ}}\left({ }_{\Lambda}^{4} \mathrm{He}\right)$ & 0.017 & 1.10 \\
\hline$\Gamma_{p}\left({ }_{\Lambda}^{4} \mathrm{He}\right)$ & 0.014 & -2.72 \\
\hline$\Gamma_{n}\left({ }_{\Lambda}^{4} \mathrm{He}\right)$ & 0.000017 & -0.061 \\
\hline$\tau\left({ }_{\Lambda}^{4} \mathrm{He}\right)$ & 0.0093 & 0.0040 \\
\hline$\Gamma_{p}\left({ }_{\Lambda}^{5} \mathrm{He}\right)$ & 0.059 & 3.48 \\
\hline$\Gamma_{n}\left({ }_{\Lambda}^{5} \mathrm{He}\right)$ & 0.161 & -3.65 \\
\hline$\tau\left({ }_{\Lambda}^{4} \mathrm{H}\right)$ & 0.023 & -0.0051 \\
\hline$Q^{-}\left({ }_{\Lambda}^{4} \mathrm{H}\right)$ & 0.160 & 3.07 \\
\hline
\end{tabular}

Table 5.6 The contributions to the error estimates for the calculations of (5.7) and using present experimental results. 


\section{PHYSICS RESULTS}

-

○

-

- 


\section{THE ISOSPIN ANTIQUARK DOUBLET IN SU(2)}

In the $\mathrm{SU}(2)$ flavor model of the light quarks consists of a doublet $\left(\begin{array}{l}u \\ d\end{array}\right)$ and a singlet $(s)$. The antiquark equivalents are $\left(\begin{array}{c}\bar{d} \\ -\bar{u}\end{array}\right)$ and $(\bar{s})$. The relation of the quark and antiquark doublet is fundamental to deriving the isospin structure.

The minus sign in the antiçuark doublet originates from transformation properties in the $\mathrm{SU}(2)$ model

$$
\mathcal{P}_{\alpha} q_{i}=\sum_{j} \mathcal{D}(\alpha)_{i j} q_{j}=e^{-i(\sigma \cdot \alpha)_{i j} / 2} q_{j}
$$

where $\mathcal{D}(\alpha)$ is a representation of the transformation $\mathcal{P}_{\alpha}$, and $\alpha$ is the parameter of the transformation. Likewise, the antiquark will transform as the complex conjugate

$$
\overline{\mathcal{P}}_{\alpha} q_{i}^{*}=\sum_{j} \overline{\mathcal{D}}(\alpha)_{j i} q_{j}^{*}=q_{j}^{*} e^{i(\sigma \cdot \alpha)_{j i} / 2}
$$

The antiquarks form a parallel isomorphic representation of $\mathrm{SU}(2)$ and is related to the quark representation by a similarity transformation

$$
q=R q^{*}
$$

and the inverse

$$
q^{*}=\mathcal{R}^{\dagger} q
$$


Combining this information yields

$$
\begin{aligned}
\overline{\mathcal{P}}_{\alpha} q_{i}^{*} & =\sum_{j} \mathcal{R}_{j i} \mathcal{P}_{\alpha} q_{j} \\
& =\sum_{j k} \mathcal{R}_{j i} \mathcal{D}(\alpha)_{j k} q_{k} \\
& =\sum_{j k l} \mathcal{R}_{j i} \mathcal{D}(\alpha)_{j k} \mathcal{R}_{k l} q_{i}^{*} \\
\text { or } \overline{\mathcal{D}}(\alpha) & =\mathcal{R}^{\dagger} \mathcal{D}(\alpha) \mathcal{R}
\end{aligned}
$$

For small $\alpha$ this can be expressed as $-\bar{\sigma}=\mathcal{R}^{\dagger} \bar{\sigma} \mathcal{R}$. It is easy to be convinced that $\mathcal{R}=\sigma_{y}$ satisfies this relation where $\sigma_{y}$ is the only imaginary Pauli matrix in the usual representation $\sigma_{y}=i\left(\begin{array}{cc}0 & -1 \\ 1 & 0\end{array}\right)$. The minus sign in the antiquark doublet is now evident from equations (A.3) and (A.4). 


\section{BIBLIOGRAPHY}

[1] Peter D. Barnes and Gregg B. Franklin. AGS Research Proposal Status Report E-788. 1987.

[2] G. P. Yost and et al. Phys. Lett. B, 204, 1, 1988.

[3] J. Cohen. Progress in Particle and Nuclear Physics, 25, 1990.

[4] W.M. Alberico et al. Phys. Lett., B256, 134, 1991.

[5] W. Cheston and H. Primakoff. Phys. Rev., 92, 1537, 1953.

[6] M. Danysz and J. Piewski. Phil. Mag., 44, 348, 1953.

[7] R.D. Grace. Weak Decay of P Shell Lambda Hypernuclei. PhD thesis, Carnegie Mellon University, 1986.

[8] John Szymanski. The Weak Decay of Lambda Hypernuclei. $\mathrm{PhD}$ thesis, Carnegie Mellon University, 1987.

[9] M. M. Block and R. H. Dalitz. Phys. Rev. Lett., 11, 1963.

[10] John F. Dubach. Nucl. Phys., A450, 71, 1986.

[11] B. H. J. McKellar and B.F. Gibson. Phys. Rev., C30, 322, 1984.

[12] D. P. Heddle and L. S. Kisslinger. Phys. Rev., C33, 335, 1986.

[13] R. E. Phillips and J. Schneps. Phys.Rev., 180, 1309, 1969.

[14] M. M. Block and et al. Nuovo Cimento, 28, 299, 1963.

[15] J. J. Szymanski and et al. Phys. Rev., C43, 849, 1991. 
[16] F. J. Gilman and M. B. Wise. Phys.Rev., 20D, 2392, 1979.

[17] C.B. Dover. Few-Body Systems, 2, 77, 1989.

[18] Commins and Bucksbaum. Weak Interactions of Leptons and Quarks. Cambridge, 1983.

[19] R.A. Schumacher. International Symposium on Hypernuclear and Strange Particle Physics, Shimoda, Japan, , 1991.

[20] B. Bassalleck. CMU/ME, 014/88, 1988.

[21] Press Flannery Teukolsky and Vetterling. Numerical Recipes. Cambridge, 1985.

[22] Savitzky-Golay. Computers in Physics, 4, 669, 1990.

[23] C. Cernigoi et al. Experimental Results on Single Nucleon and Correlated Pairs Emitted after Negative Pion Absorption at Rest in Light Nuclei. In Perspectives in Nuclear Physics at Intermediate Energies, page 212, 1983.

[24] J. J. Szymanski and et al. Phys. Rev., 49, 1991. 

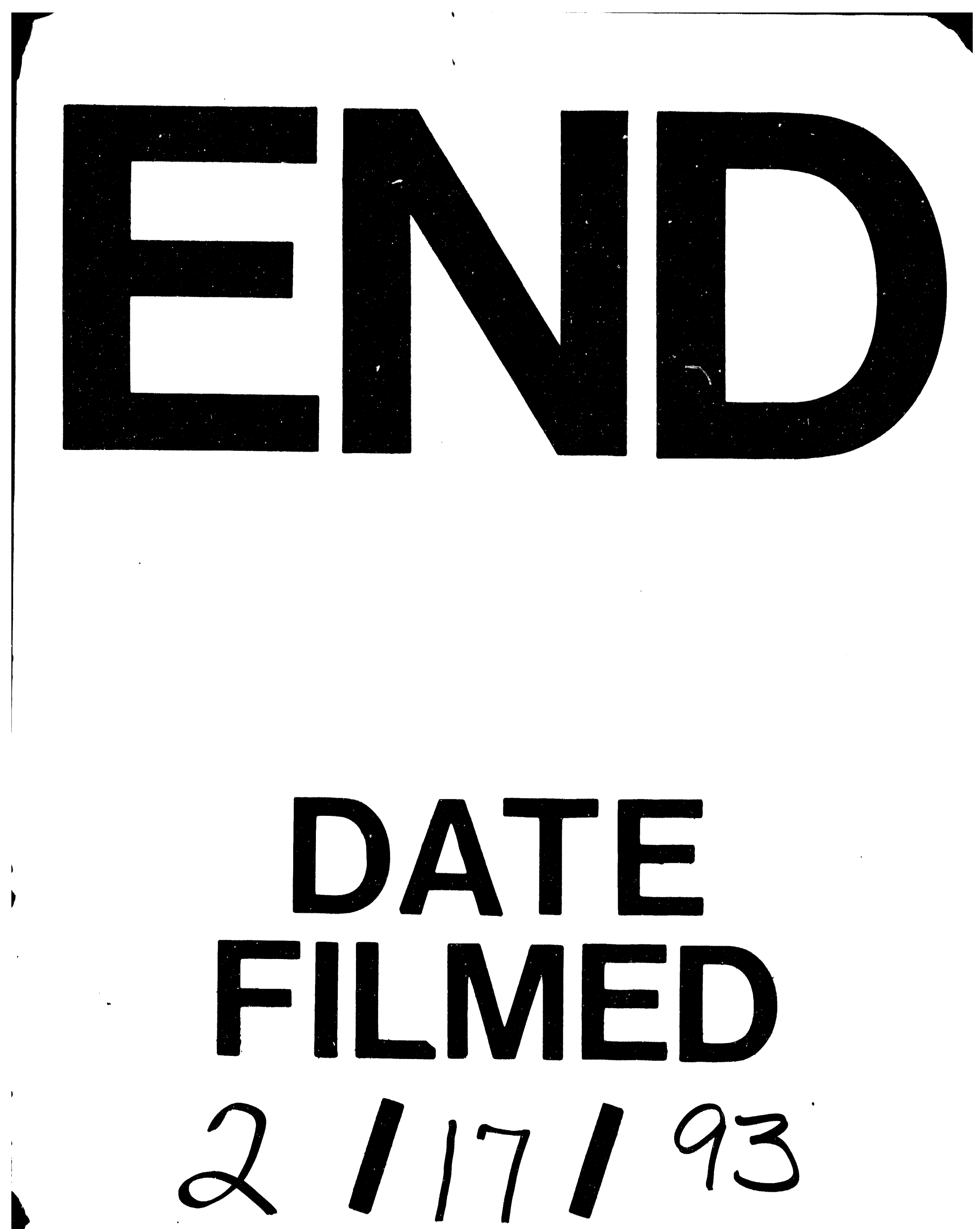

93 


$-$

